Portland State University

PDXScholar

$12-17-2019$

\title{
Systems Isomorphisms in Stochastic Dynamic Systems
}

Rajesh Venkatachalapathy
Portland State University

Follow this and additional works at: https://pdxscholar.library.pdx.edu/open_access_etds

Part of the Psychology Commons, and the Sociology Commons

Let us know how access to this document benefits you.

\section{Recommended Citation}

Venkatachalapathy, Rajesh, "Systems Isomorphisms in Stochastic Dynamic Systems" (2019). Dissertations and Theses. Paper 5410.

https://doi.org/10.15760/etd.7283

This Dissertation is brought to you for free and open access. It has been accepted for inclusion in Dissertations and Theses by an authorized administrator of PDXScholar. Please contact us if we can make this document more accessible: pdxscholar@pdx.edu. 
Systems Isomorphisms in Stochastic Dynamic Systems

by

Rajesh Venkatachalapathy

A dissertation submitted in partial fulfillment of the requirements for the degree of

Doctor of Philosophy

in

Systems Science

Dissertation Committee:

Martin Zwick, Chair

Tom Seppalainen

Thaddeus Shannon

Marek Perkowski

Portland State University

2020 


\section{Abstract}

The identification of isomorphisms between disparate systems is an important focus of systems science. Such isomorphisms have not only conceptual and pedagogical value to systems science, but they also provide pragmatic value to specific disciplines by suggesting new ways to model familiar phenomena and by serving as reference models that show how even simple models can generate complex behavior. Specifically, this dissertation looks at certain classes of stochastic dynamic systems (SDS) and shows that similar equations can model phenomena in sociology and psychology. In both disciplines, what is modeled by these SDS isomorphisms is a certain type of reliability, defined as the satisfaction of constraints, expressed in terms of first passage (exit) times to boundaries.

In mathematical sociology, the work revisits older literature on Markov models of occupational mobility and generalizes it to show how SDS can model intragenerational mobility and escape from poverty traps. By looking at exit-times and exit-probabilities of escape from such traps, it points out features of social dynamics, such as transients, which are often missed by equilibrium macroeconomic and macro-sociological models.

In mathematical psychology, the work looks at literature on drift-diffusion models (DDM) of time constrained judgment and decision making. Inspired by models of escape from attractor and stochastic switching dynamics in simple neurophysiological processes, it proposes extensions of DDM, again illustrating the 
role of exit-times and exit-probabilities. These models could serve as null reference models for experiments in cognitive psychology and motivate new experiments.

From the highly biology-centered systems science perspective, the probability distribution (and its moments) of the first exit out of a region in state space has a natural biosemiotic interpretation in terms of viability or lack thereof. Potential applications to modeling biological behavior are also sketched in this work. The SDS approach connects with Ashbys law of requisite variety, Simons concept of satisficing, and Holling's dynamic systems idea of resilience. Thus in addition to offering new ways to model phenomena in the social sciences and biology and suggesting new mathematical and scientific questions worth pursuing, the models developed in the dissertation add to the repertoire of significant systems isomorphisms, continuing the tradition of Ashby's Introduction to Cybernetics and Zwicks forthcoming Elements and Relations. 


\section{Contents}

Abstract

List of Figures $\quad$ vi

1 Introduction 1

1.1 Systems isomorphisms and their role in scientific discovery . . . 6

1.2 Motivation . . . . . . . . . . . . . . . . 8

1.2.1 Biological Motivation . . . . . . . . . . . . . . . 9

1.2 .2 Systems Science . . . . . . . . . . . . . . . . . . 17

1.3 Systems approach to questions and solutions . . . . . . . . . 18

1.4 Models of Reliable Behavior . . . . . . . . . . . . . . . . 20

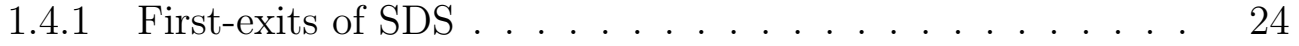

1.4.2 From first-exits to reliability . . . . . . . . . . . 28

1.5 Micro-nano physiology: processes and models . . . . . . . . . 31

1.6 Applications . . . . . . . . . . . . . . . . . . . . . . 35

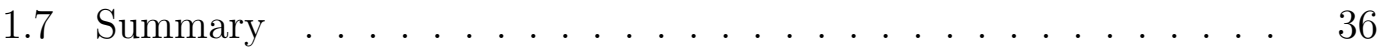

2 Revisting Markov models of Intragenerational Mobility 38

2.1 Introduction . . . . . . . . . . . . . . . . . 38

2.2 Social Mobility: Literature and Models . . . . . . . . . . . . 42

2.3 Models of Intragenerational Mobility _. . . . . . . . . . . 44 
2.3.1 First-passage time models of escape from poverty . . . . . 51

2.3.2 MFPT under different conditions . . . . . . . . . . 53

2.3.3 From one dimensional first-passage time problems to escape from poverty and intragenerational mobility . . . . . . 58

2.3.4 Modeling mobility dynamics and escape from poverty traps 62

2.4 Conclusion . . . . . . . . . . . . . . . . . . . . . 69

\section{Drift-Diffusion Models of Bias, Uncertainty and Conflicts in Judg-} $\begin{array}{ll}\text { ment and Decision Making } & 72\end{array}$

3.1 Introduction . . . . . . . . . . . . . . . . . 72

3.1.1 Connections to models of Chapter 2 . . . . . . . . 77

3.2 Models . . . . . . . . . . . . . . . . . . . . . . . . 79

3.2.1 Classical DDM . . . . . . . . . . . . . . . . . 83

3.2.2 Models with stochastic drift . . . . . . . . . . 88

3.2.3 Models with non-trivial deterministic drift . . . . . . . . . 91

3.2.4 Applications ..................... 93

3.3 From DDM to Ecological Rationality . . . . . . . . . . . . . 98

3.4 Conclusion ...................... . . 101

$\begin{array}{lll}4 \text { Conclusion } & 107\end{array}$

4.1 Summary of contributions and future work: Mathematical Sociology 109 4.1.1 Additional insights . . . . . . . . . . . . . . . 110

4.2 Summary of contributions and future work: Mathematical Psychol-

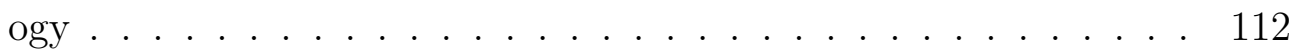

4.2 .1 Additional Insights . . . . . . . . . . . . . . . . . . . . . 114

4.3 Pragmatic value of these SDS systems isomorphisms . . . . . . . 116 
4.3.1 Gaps and connections in scientific literature identified by SDS isomorphisms . . . . . . . . . . . . 118

4.3.2 Gaps and connections in the mathematical literature identified by SDS isomorphisms . . . . . . . . . . . . 119

4.4 Scientific explorations suggested by these systems isomorphisms . 121

4.4.1 More general models of escape from poverty . . . . . . . . 122

4.4.2 More variants of DDMs . . . . . . . . . . . . . . . . . . 123

4.4.3 Animal Behavior ... . . . . . . . . . . 125

4.5 Mathematical explorations suggested by these systems isomorphisms 127

4.5.1 Duality between Optimization and Constraint Satisfaction 128

4.5.2 Inverse problem approach for general stochastic dynamical systems . . . . . . . . . . . . . . . . 130

4.6 Conclusion . . . . . . . . . . . . . . . . . . . . . . 132

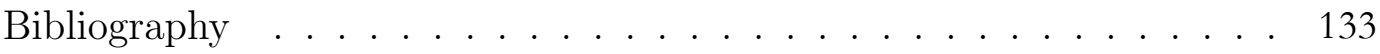




\section{List of Figures}

1.1 Conceptual diagram illustrating the idea of lifting and systems iso-

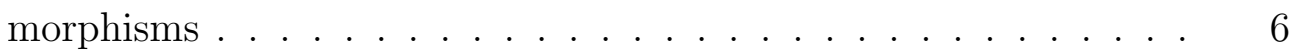

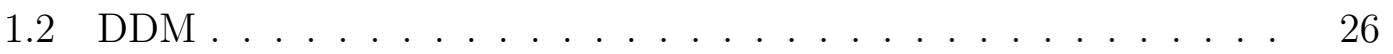

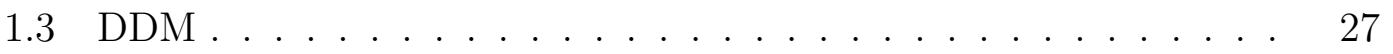

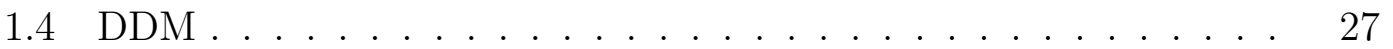

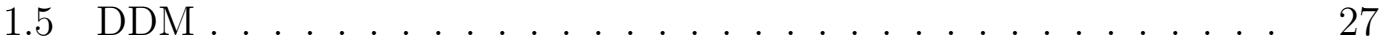

2.1 Diffusion on an infinite line . . . . . . . . . . . . . . 48

2.2 Diffusion with drift on an infinite line . . . . . . . . . . . 49

2.3 First passage time density for varying diffusion constants . . . . 50

2.4 MFPT for diffusion on an interval . . . . . . . . . . 54

2.5 MFPT for diffusion on an interval . . . . . . . . . . . 55

2.6 Conditional MFPT diffusion with drift on an interval . . . . . 56

2.7 Model of poverty traps . . . . . . . . . . . . . . . . . . . 62

2.8 Model of poverty traps with a small drift component . . . . . . . 63

2.9 Model of poverty traps with a moderate drift component . . . . . 63

2.10 Model of poverty traps wirth large drift component . . . . . . . . 64

2.11 Poverty dynamics in the presence of health failures $\ldots \ldots$. . . 66

3.1 Drift-Diffusion Model . . . . . . . . . . . . . . . . . . . . . . 84

3.2 DDM with (almost) pure diffusion starting at the center $\ldots . .85$ 
3.3 DDM with (almost) pure diffusion starting at the center . . . . . 86

3.4 DDM with (almost) positive and negative drift . . . . . . . . . . . 87

3.5 DDM with positive drift of increasing magnitude . . . . . . . . 87

3.6 DDM with positive drift of increasing magnitude . . . . . . . . . . 90

3.7 DDM with nonlinear deterministic drift . . . . . . . . . . . . 91 


\section{Introduction}

This dissertation is motivated by a desire to understand the surprisingly deterministic behavior of agents in the presence of stochasticity, especially the reliability of this behavior. The general nature of this behavior requires a study centered around general principles. This makes it an ideal candidate for a systems approach. After constructing a specific notion of reliability, we find isomorphisms between social and psychological processes that demonstrate how this same notion of reliability can be used in sociology to understand escape from poverty traps, and in psychology to understand decision making. Along the way, we discover connections within related sub-fields in sociology and psychology, illustrating the utility of systems approach in scientific inquiry and mathematical exploration.

Systems isomorphisms are distinct from more pervasive interdisciplinary isomorphisms. Interdisciplinary isomorphisms arise out of tools shared by epistemic communities; such isomorphisms have a serendipitous characteristic and are typically surface level similarities. Systems isomorphisms are more general but also of a specific kind. Any given systems idea involves an interplay of three aspects: a conceptual aspect, a scientific aspect and a mathematical aspect. A scientific concept, via a mathematical formalization, is generalized at the conceptual aspect. The newly generalized concept can now be used for scientific inquiry in other disciplines. Models resulting from such inquiries in different disciplines share similarities. These model similarities are the systems isomorphisms. 
The conceptual aspect anchors any particular systems point of view. In the highly biology-centered perspective [137], the conceptual aspect is based on an assortment of ideas emphasizing biological semantics and the biological level. However, this work prefers a more mathematically precise version. Here, the conceptual aspect is anchored around a generalized biology. In this point of view, the conceptual aspect is more abstract than the scientific aspect. It carries with it a general biology like semantics. The mathematical level is more abstract than the conceptual aspect with no semantics associated with it. In going from a scientific aspect to a conceptual aspect, a scientific aspect can be thought of as being lifted from the scientific domain and generalized at the conceptual level. By lifting a scientific concept and treating it as a systems concept, models associated with the original scientific concept are available to be used in disciplinary scientific inquiry in fields unrelated to the original scientific concept. The isomorphisms identified in this work have this genesis. The above process of lifting is visualized in Figure 1.1 .

The approach presented here, especially the idea of lifting, is aligned with Bunge's conception of systems philosophy [27] as an exact and scientific metaphysics. As will be discussed subsequently, the dissertation aspires to lift biological reliability to an exact metaphysical concept, in the sense of Bunge. In doing so, we illuminate the importance of constraint satisfaction, more specifically a temporal version of constraint satisfaction, discussed in disparate disciplines under various guises. A successful synthesis of a systems idea does not guarantee the applicability of such a systems idea in every scientific domain. As Bunge argues [27], Popperian refutability is not applicable to systems theories; either an idea is useful in a specific scientific context, or it is not.

The concept that is generalized here is a type of reliability of biological be- 
havior. The first step of lifting to the systems aspect is broken down into two smaller steps. In the first, by appealing to connections between physiology and behavior, one can reinterpret certain characteristics of nanoscale and microscale physiological processes and the mechanisms underlying them as being reliable, especially the ability of these processes to generate low failure rate behavior, even under high ambient stochastic conditions. These micro and nano scale processes are modeled by a certain class of stochastic dynamical systems (SDS). First-exits or first-passage times of these SDS are adequate descriptors of transient characteristics of these biological processes [66]. For example, in the dendritic spines of neurons [54] -projections on the cell body that serve as chemical isolation compartments, $\mathrm{Ca}^{+2}$ ions are trapped and isolated from the rest of the neuron. When a neuro-transmitter molecule docks onto these spines, the trapped ions are released through a narrow-exit. The precise geometry and topology of the spine determine the typical time it takes for the ions to escape out of these spines. Such release timescales are highly deterministic in spite of the stochastic nature of sub-cellular environments. Over the course of an organism's life, these spines change their shape, tuning their time constants to match both upstream and downstream biological processes. These characteristics display a certain kind of determinism that is surprising given the highly stochastic environment in which the processes take place. This determinism has biological significance for other physiological processes that happen downstream. Biophysical and biochemical parameters driving the processes seem to be fine tuned to produce this same surprising ${ }^{1}$ determinism, all without the use of control modules, and only with constraint satisficing. In the

\footnotetext{
${ }^{1}$ In the disparate disciplines focused on explaining the macro from the micro, emergence of determinism from underlying stochastic is of interest. While it is easy to see determinism emerge from microscopic randomness in physical systems, the emergence of determinism at the meso-scale - the level of physiological processes, from stochastic processes at the same scale, or at lower levels, is novel, and hence surprising.
} 
second step, by identifying reliability with these characteristics, the characteristics of behavior at higher levels can be modeled.

Though the biological conception of reliability is more important for this dissertation, models of reliability conceived and often used to characterize engineering structures serve as template for the mathematical models of biological reliability presented in this dissertation. Reliability is quantified in a variety of ways in engineering: some focus on risk assessment; some on functional readiness of systems; some on fault tolerance, and many others. This dissertation identifies reliability with the quantification of time-dependent failure probability. Such measures of reliability continue to be used in the analysis of reliability of engineering structures, especially to study forms of mechanical vibrational instabilities under environmental stochasticity [127].

Models of these mechanical structures are based on nonlinear stochastic systems. Time evolution of such systems is calculated as solutions of Fokker-Planck equations. In all cases, a multidimensional nonlinear dynamical system is assumed to be perturbed by Gaussian noise. In such mechanical systems undergoing oscillations, the time it takes for the oscillation to cross a certain threshold is an important empirical quantity important for objectively characterizing the system's fault tolerance. These empirical measures are often identified with statistics of first-passage time of the stochastic model, and the same measures are used to quantify temporal characteristics of micro and nano physiological processes discussed previously.

This generalized concept of reliability at the systems level comes with mathematical models of reliability based on SDS (Section 4 of this chapter). This is then used for scientific inquiry in sociology (Chapter 2) and psychology (Chapter 3). In sociology, the concept of reliability is associated with dynamics in and 
out of poverty traps. In psychology, the concept of reliability is associated with dynamic judgment and decision tasks. The systems isomorphism identifies similarities between social and psychological processes of wealth accumulation and evidence accumulation. The isomorphism between these two processes extends to the notion of fine tuning and constraint satisfaction, both lifted from physiology. In sociology, fine tuning and constraint satisfaction are related to questions of interest to a social policy maker; specifically, reliability is associated with escape from such traps. In psychology, fine tuning and constraint satisfaction are related to questions about satisficing decision and judgment heuristics; specifically, reliability is associated with the successful execution of such judgments and decisions. In sociology, policy makers may seek to fine tune the environment to produce reliable escape from poverty traps. In psychology, the decision making modules may fine tune the parameters underlying the dynamics of evidence accumulation to produce reliable judgments or decisions. While not discussed in greater detail like other applications in this work, the isomorphisms extend to models of higher behavior of artificial and natural agents (Chapter 4). 


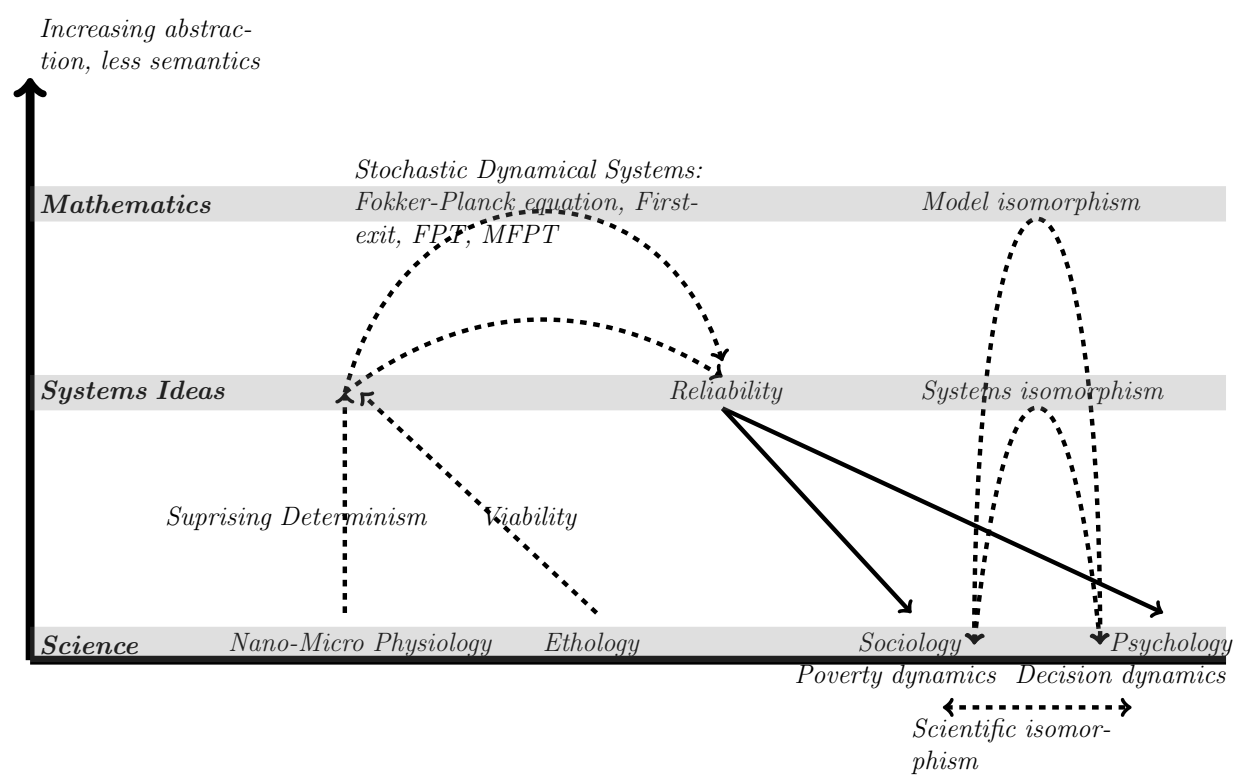

Figure 1.1: Conceptual diagram illustrating the idea of lifting and systems isomorphisms

\subsection{Systems isomorphisms and their role in scientific discovery}

While a systems approach can be justified on purely philosophical, conceptual and aesthetic grounds [137], it can be argued to be part of conventional scientific discovery processes. Different perspectives on this process have been offered.

Hofstadter [65] emphasizes the role of analogical reasoning in scientific discovery. Simon $[120,81]$ offers a computational take, and works from foraging model of scientific discovery. Holland and Thagard [68]build on Simon's perspective and the concept of coherence to develop a model of induction. Along with these older positions, modern enterprises like machine learning find transfer learning to be a key concept underpinning many useful artificial systems. Taken together, these models of scientific discovery require search and recognition heuristics that are coherent ${ }^{2}$. They are meant to capture consistency criteria among scientific

\footnotetext{
${ }^{2}$ The notion of coherence is discussed in [68]. The concept as envisioned by Thagard is a way to seek compatibility of scientific knowledge acquired through inductive, abductive and
} 
explanations, theory induction and other steps during scientific knowledge accumulation [68]. Such heuristics allow a transfer of concepts and models from one domain to another. Systems isomorphisms can be simply thought of as belonging to such a family of heuristics important for scientific inquiry but only more general. Pragmatically, a wider and richer model class is available for inquiry when we use a systems approach.

Although systems isomorphisms are often biological in origin - as is the case for the physiological nano/micro models drawn upon here - the applications of these isomorphisms are not restricted to biological domains. In this sense, systems isomorphisms can connect different disciplines not usually considered similar to one another. While an ant colony, an operating system and nervous systems may be materially different, the essential similarity of their information processing nature makes these systems comparable. This is the spirit behind the identification of isomorphisms in this dissertation.

Unlike interdisciplinary research which typically links adjacent disciplines (such as physics and chemistry or chemistry or biology), often via reduction, a systems approach finds isomorphisms from a range of disciplines. In the present work, the fact that psychology and sociology might be viewed as adjacent disciplines is in no way involved in the use of the same mathematical models in both. Having identified shared characteristics of all these systems, discussions of generalities often but not always involve the use of biology-like categories. Reasoning and explanations using these generalized biological categories provide insight for the specific systems in a disciplinary context. For example, using insights in collective animal behavior in ants [59], models and mechanisms of how immune cells respond to foreign objects have been built [60]. The same models have been used to develop deductive means. More coherence a theory or model possesses, the more constraints it satisfies. 
distributive detection algorithms, and have provided insights for collective human behavior.

In this dissertation, we identify isomorphisms in certain classes of stochastic dynamical systems (SDS), construct a notion of reliability as satisfaction of constraints, and express them as first-passage time to a boundary. The probability distribution (and its moments) of the first exit out of a region in state space has a natural biosemiotic interpretation: an interpretation of state space as some kind of adaptive behavior in the context of the behaving agent's cognitive ecology, and ethological importance.

We work with this biosemiotic interpretation, unpack consequences for specific disciplines (here sociology and psychology, Chapter 2 and 3 respectively), and demonstrate how this systems approach generates scientific insights for specific

disciplines (Chapters 2, 3 and 4). This is the precise sense in which a systems approach adds value to specific scientific inquiry.

Below, first, we present core ideas that motivate this work; then, present the model of reliability; and conclude by summarizing the contributions of this dissertation.

\subsection{Motivation}

Biological motivations of this dissertation lie in ethology and physiology. A reverse engineering orientation allows us to formalize the specific concept of reliability using systems ideas of Simon, Ashby and Holling. The dynamic nature of behavior, together with stochasticity, leads us to mathematical theories of stochastic dynamical systems which we discuss in section 4. 


\subsubsection{Biological Motivation}

Two ideas from physiology and ethology are of interest here. In physiology, nanoscale and microscale processes demonstrate surprising determinism in their temporal characteristics in spite of ambient stochasticity. In ethology, at meso and macro scales, reliable behavior has a biosemiotic salience and shares similar deterministic characteristics, especially in the behavioral primitives underlying complex sequences of behavior.

\section{Physiology}

In On Growth and Form [128], Thompson sketched an outline for applied mathematicians and biologists, inviting them to construct a scientifically rigorous theory of morphogenesis: the study of how forms arise in biology. This entails that one acquires an understanding of how genes and biological macromolecules use ambient substrate in an environment to produce a specific stable (reliable) shape in the presence of uncertainty.

The morphospace, the space of all possible shapes, is a very high dimensional space; if one wanted to enumerate these possibilities, their number would be extremely large. However, biology seems to operate on a much lower dimensional space. Research exploring reasons for such a collapse in dimensions have begun to show [82] how a potentially low-dimensional state space emerges, and points to the role of environmental constraints in enabling this collapse of dimensions. Also, this collapse is not an active process -no controls guiding this collapse. This is consistent with what is known about biological processes; they typically operate at a narrow set of possibilities. In subsequent sections and in chapters 2 and 3 , this fine tuned nature of biological processes is further elaborated. Since in many 
biological scenarios, shape modulates and makes biological function possible, the connections to physiology are more than speculative [82].

Patterns are generally associated with regularities in space. However, regularities are possible not only in space but also in time. Patterns can be abstract, or can be chemical or electrical in nature. If morphology is the study of static patterns, then some aspects of physiology can be interpreted as the study of dynamic

patterns. Such a generalized pattern constrains and enables biological function, and it is in this sense that physiology and behavior are similar. Just as such morphology is the scientific study of form, physiology could be envisioned as a study of generalized spatio-temporal form. And just as morphogenesis involves the interaction of the internal and the external, physiology also involves similar interactions. And constraints have a similar role to play in both morphology and physiology, especially in how it enables reliability. Most importantly, the collapse of dimensions and matching of parameters to enable function seen in morphology has analogs in physiology. This has implications for the work presented in this dissertation.

Recent studies have uncovered details of complex physiological processes at the nano and micro scale. Common to many physiological processes at those scales seem to be a certain recurrence of timing based sequence of events. This implies a certain capacity for the system to exhibit partially deterministic behavior in spite of the highly stochastic exogenous and endogenous conditions.

\section{Nano and micro physiology}

Diffusive particles play a key role in both cellular and molecular biology $[115,67]$. The particle trajectory, typically modeled as a Brownian motion, can represent the motion of molecules, proteins, ions, receptors and various cellular organelles 
present in cellular membranes and in cellular cytoplasm. In all these cases, the mean time to reach a target while avoiding obstacles is a key regulator of cellular function. The cell's function or behavior is in response to environmental regularities and the precise relationship between these regularities and internal physiology is of interest to biologists.

Even though the physics of the system is known in principle via Netwon's laws and Maxwell's equations, the peculiarities of biological matter, the way it is arranged spatially and the nature of biomolecules that make up the system, leads to emergence of novel equations $[66,67]$ at these small spatial and temporal scales. Not only that, chemical kinetics, which is usually defined for large numbers of reacting species, breaks down for small numbers, requiring modifications of their own. Also critical is the role of geometry and topology of the domain in which these processes take place.

Apart from escape of $\mathrm{Ca}^{+2}$ ions out of dendritic spines discussed elsewhere [20], other examples include stochastic molecular search mechanisms in the cytoplasm, receptor docking mechanisms on cell membranes, cytoplasmic viral trafficking, endosomal viral escape and diffusive transfer of genetic material during cell divisions. In all these cases, the mean time to an event is critical, whether it is for ion transport or viability of virus inside a cell. The time constant matches some aspects of temporal regularities of the environment, enabling function ${ }^{3}$.

Similar processes are relevant in understanding neural excitability [54]. Robust and stable time constants drive processes at synapses, axons and bio-chemically localized sub-cellular compartments. In many cases, the time constants are dependent on diffusion type transport of macromolecules and ions within the cyto-

\footnotetext{
${ }^{3}$ In all these cases, the models of these biological processes make use of stochastic dynamical system theories, solutions of Fokker-Planck equations, and first-passage time analysis
} 
plasm, towards the cell membrane; some processes rely on extra-cellular diffusive transport and movement on the membrane. Such processes are seen in neurophysiological processes at the same scales.

In neuroscience, especially in the literature on neural microcircuits [117, 63], the importance of such time constants and their implications for downstream biological function is beginning to be discussed [117, 63]. Also, while diffusion induced neural spiking and temporal coding is salient in the neural encoding literature and is discussed in the context of cognitive neuroscience, its relevance for neuro-ethology is not explicitly discussed. The focus on encoding decoding mechanisms and neural representations makes neuroscience biased towards cognitive aspects of neural substrate -it pushes aside questions that are relevant for biological function aside. Importance of reliability and biophysical (neural) processes enabling it are discussed as such in ethology and neuro-ethology, especially in the studies of neural microcircuits underlying insect locomotion and escape response $[54,117,63]$. Since the models in this dissertation are behavioral, discussions of relevant neuro-ethology are neglected; instead, focus is on whole animal behavior (ethology) with special attention towards reliability.

The nature of the environmental regularity or irregularity has some shared characteristics across scales spanning macro and meso level all the way to micro and nano levels: they are stochastic; the environment possess non-trivial but regular topological and geometric feature; and possess clear timescales. This suggests that the same models that are useful in nano and micro physiology are potentially useful also in meso or macro physiology, i.e., the subject of ethology. This is not because higher level phenomena are reducible to lower level phenomena, but rather because isomorphisms exist that make phenomena at both levels similar. 


\section{Ethology}

Although very old, Tinbergen's vision for ethology [129] is still a good starting point for talking or researching principles about animal behavior. His Four Questions are

- What is its physiological causation?

- What is its function or survival value?

- How has it evolved over time?

- How has it developed in the individual?

Shettleworth [118] summarizes these four questions to be about cause, function, evolution and development respectively and added fifth question on intralevel and interlevel integration of different disciplines answering these Four Questions. Webb [132] expands the discussion to include behavior of artificial agents and is related to an early work by Brooks [25]. And finally, focusing on higher organisms, Bullock's work on comparative neuro-ethology $[26]^{4}$ emphasizes diversity of neural mechanisms that generate behavior.

Animal behavior is typically documented using ethograms [71], a recording of behavioral repertoires decomposed into behavioral primitives. An understanding of these primitives is of general interest to ethologists. These behavioral primitives can be mathematically characterized and modeled. Among the various observed characteristics, a behavior's reliability is the core focus of the dissertation. And this reliability is often defined by time-to-event constraints that need to be satisfied

\footnotetext{
${ }^{4}$ Biological explanations can be structural, functional or historical. Neuroscience mostly focuses on structural explanations, while neuro-ethology focuses on structural explanations with function in mind.
} 
in the presence of stochasticity. In the rest of this discussion (in this chapter and in Chapter 4), we restrict ourselves to two such primitives.

Escape behavior is evasion in response to typically harmful environmental signal. Startle response is an abrupt and fast behavior and may be building blocks of more complex startles. From a modeling point of view, we can ignore the subtle differences between escapes and startles and in what follows focus on startles, treating escapes to be conceptually synonymous. Fixed action patterns, on the other hand, are also triggered by environmental cues, but are spatio-temporally complex and longer timescale behavior.

Consider an organism with or without a nervous system whose body size and weight are small enough so that environmental fluctuations induce instability in the organism's movement. Suppose the organism is exposed to sudden harmful stimulus. In response to this environmental signal, the organism must reliably escape or respond to avoid consequences. Adaptation to exposure to harmful signals may occur evolutionarily, developmentally or over its life history. Similarly, consider a situation where the organism, upon presentation of a cue, is expected to locomote in space. This locomotion, a fixed action pattern (FAP), requires the system repeat its body configurations over an extended but finite period of time, in a stochastic environment. The disturbance may wobble and destabilize locomotion but not by much.

In the case of escape and startles, ideal behavior is one that completes the act by a definite time. In the case of FAP, ideal behavior is one that maintains the pattern for a definite time. Both primitives involve time constants; in case of startles and escape, it is time to viability; in the case of FAP, it is persistence of viability. It is in this sense that both the processes guaranteeing reliability at the nano and micro scale are related to behavioral primitives at the meso and macro 
scale.

Typically, both these kinds of behaviors are modeled either using optimality principles or are built ad-hoc to reproduce behavioral templates. A key question is the source of reliable behavior under varying conditions of internal and external stochasticity. Reliable startle behavior involves movement of the body of the organism in an environment; it involves avoiding a subset of space of possible configurations while reaching a subset of final configurations within a fixed time. Reliable fixed action pattern involves repetitive movement of the body in an environment while being subjected to uncertainty in conditions both internal and external to the organism. Also,reverse engineering explanations make use of control theory based modules to explain reliability. Instead, we find constraint satisfaction simpler and adequate in many cases. The models of reliable behavior discussed in section 4 is based on expectation valued measures and is routinely used in the literature studying reliability of engineering structures. Stronger forms of reliability require advances in mathematics behind these models. This issue is addressed in chapter 4 .

The particular dynamic systems type of reliability considered in this work differs from the conventional type of reliability which is defined control-theoretically and studied in the traditional engineering disciplines. Our focus on dynamic systems reliability can be justified biologically [26], and it can also be justified by an argument similar to that of Brooks[25], who denied the need for representation in control. One can instead think of behavior as being generated by making use of internal representations. One potential remedial strategy might be to think of behavior as being generated by merely a biological substrate. Once can call such a lower form of internal process physiological, as opposed to cognitive; and can correspondingly consider the dynamic systems type of reliability discussed in 
this work as also lower than the type of reliability that is achieved via control modules. This work does not seek to replace the cognitive, it merely suggests that some aspects of control can be regained with a lower form of behavior. We illustrate this idea with two examples.

Closure of Venus flytrap leaf [49] is an uncharacteristically fast movement among plants. It is especially interesting given the lack of a muscular system or a nervous system. The closure of the trap is triggered by stimulation of hairs in the trap but the post-stimulation movement is a result of mechanical instability. The rapid closure is not controlled but dynamics arising out of the physics of the system and is a result of the system entering an instability phase. The parameters of the system are fine tuned to be at the edge of this bifurcation instability.

Another example is the crawling locomotion of soft-bodied animals [96]. While such repetitive behavior is usually generated by central pattern generators (CPG), evidence for CPG in organisms like earthworms and insect larvae is weak. In such cases, a neuromechanical model that enables rhythmic movement by coupling the neuro-muscular dynamics with the environment, via friction generated by the body, is able to generate full organism coordination of patterned crawling. In this example, even if nervous system is involved, the locomotion is not due to precisely controlled neural circuits. It is because of fine tuning of parameters of the coupled dynamics.

Both these examples illustrate one of the main points of the dissertation: fine tuning without constraint satisfaction -under some circumstances- replace the need for control modules.

Further discussions of these notions require the generality provided by systems approaches. While related ideas are already present in the systems literature, the particular emphasis on viewing behavioral reliability through the lens of physi- 
ology has not been discussed in the literature. Next, we discuss ideas useful for understanding lifted concepts like fine tuning and reliability. Then we point out ways in which the ideas presented previously differ from extant ideas in systems literature.

\subsubsection{Systems Science}

We find four systems ideas essential to a conceptually and mathematically rigorous formulation of of the type of biological reliability that we are considering here, in biochemistry, Monod's idea [90] of chance and necessity; in decision theory, Simon's [121] concept of satisficing; in cybernetics, Ashby's [5] law of requisite variety, and in ecology, Holling's [69] idea of resilience.

Biological reliability is associated with the concept of biological viability. Reliability is a measure of an agent's ability to stay or reach viable regions of biological activity. Viability is necessary; once viability constraints are met, other dimensions in the system's state can be subject of chance, subject to constraints that make sure the system is viable.

According to Simon, biological optimality is a concept that makes sense only in the context of environment of an agent. A weaker notion of satisfaction suffices in lieu of optimization. Satisficing for reliability requires the internal structure to interact with the environment, via an interface in such a way to guarantee reliability. In some instances, constraint satisfaction is sufficient for generation of reliable behavior.

According to Ashby, biological regulation requires internal complexity to match the agent's environmental complexity. Variety in the agent's degrees of freedom must supersede that of the environment for the system to persist. Maintenance of viability or time to reach viability requires not only variety but also requires 
constraints on the structure or topology on the space of internal variety. Fine tuning and constraints on internal variety without the use of a internal control module can also generate reliable behavior.

According to Holling, biological resilience requires behavioral states to stay within a basin of attraction. Escape from a basin of attraction may also make behavior resilient by taking the system to a more desirable basin of attraction. This intrinsically dynamic notion of resilience is in contrast to the more traditional notion of stability. While control modules guarantee stability, constraint satisfaction via fine tuning can, in principle, guarantee reliability.

Notions like reliability, fine tuning, constraint satisfaction and viability required the generality of systems ideas. The work of Simon and Ashby is used to understand the conventional control theoretic points of view. However, it is possible to discuss regulation and optimality from a lower physiological point of view. This approach allows the fine tuning discussed in the context of lower physiological processes and lift it to discuss reliability of biological behavior.

\subsection{Systems approach to questions and solutions}

Motivations for this dissertation as presented in the previous section are typical of systems approach. All the aforementioned systems ideas originated in specialized contexts: Monod's ideas were a result of his work on molecular biochemistry; Simon's ideas arose in his work on administrative and organizational behavior; Ashby's ideas were originally conceived for purposes of understanding control and regular in cybernetic systems; and finally Holling's ideas were developed to understand aspects of ecological systems that were dynamical in nature. Systems approach lifts them out of their disciplinary grounding and leaves them in an 
abstract category of systems ideas.

Similarly, specific questions one is interested in can be lifted to the systems aspect. If one manages to find answers at the systems aspect, the arrived upon solutions match the generality of systems discourse, making them solutions in all disciplines.

In particular, the motivations of this work lie in developing a conception of stochastic dynamic systems type of reliability at the level afforded by systems ideas. In particular, both study of biological behavior and study of biological function underlying this behavior find generalizations at the systems level. And like other systems ideas, once we provide an exact (mathematical) formalization of the question and an associated answer, the solution is then potentially applicable in all domains. The applied domains are themselves related by systems isomorphisms.

The conception of reliability is related to Holling's notion of resilience and is based on constraint satisfaction via fine tuning and is different from ideas of Simon and Ashby. Simon's notion of satisficing says nothing about the specific implementation of an internal structure. In typical models of internal structure, control elements are used. Similarly, Ashby's law of requisite variety says that the variety of the regulator should match the variety of disturbances. While control elements optimize behavior, constraints need to be imposed. The imposing of constraints can be thought of as restricting the parameters of the internal parts to a lower dimensional space of possible. It is this restriction that we call fine tuning.

Having identified interesting ideas from nano-micro physiology and ethology, we lifted them to the conceptual aspect, elevating them to systems ideas. The processes at that scale show a certain kind of surprising determinism despite am- 
bient stochasticity. These processes are constrained by physiological structure of other background processes. Satisfying these constraints require tuning the chemical, electrical and mechanical properties of the substrate. The tuning constrains the space of the possible. Natural selection forces act like a sieve to constrain the possible to the viable. Geometry, topology and boundaries of the underlying state spaces are also constrained. In many instances, viability can be further characterized as also being reliable. The systems approach allows us to lift all this to a level of abstraction that enables application to these ideas to other unrelated systems.

In the next section, we formalize this idea of reliability of agents using stochastic dynamical systems, use first-exit techniques used in the field to formalize the notion of reliability. We then find discipline grounded phenomena in sociology and psychology that are amenable by these models.

\subsection{Models of Reliable Behavior}

An exact formalization of reliable behavior in the presence of stochasticity that unfolds in time and in space requires the model be a dynamical system, contain stochasticity, has a continuous state space, and evolve in continuous time. The

simplest possible system with all these features is a stochastic dynamical system with Brownian noise.

$$
d x(t)=v(x) d t+\sqrt{2 D} d w
$$

where

- $x$ is an one-dimensional state space 
- $D$ is the diffusion constant for Brownian noise $w$

- $v(x)$ is called the drift coefficient and can depend on the state space

The Brownian noise process or Weiner process $w(t)$ satisfies

- $w(0)=0$

- $\mathrm{w}(\mathrm{t})$ is almost surely continuous

- $w(t)$ has independent increments

- $w(t)-w(s) \sim N(0, t-s)$ for $0 \leq s \leq t$ and where $N\left(\mu, \sigma^{2}\right)$ is a normal distribution with mean $\mu$ and variance $\sigma^{2}$

If one sets $D=0$, the noise term vanishes and what one gets is a deterministic nonlinear dynamical system

$$
d x(t)=v(x) d t
$$

While this connection with deterministic dynamical systems makes the system appealing to motivate, it is not clear how to perform an analysis of such a stochastic evolution. In order to do this, one needs a mapping to a more rigorous formulation in terms of measure theoretic probability [74]. In that formulation what is more natural is the notion of Markov processes.

Markov processes are stochastic processes that satisfy the following independence relation

$$
P(\text { past }, \text { future } \mid \text { present })=P(\text { past } \mid \text { present }) P(\text { future } \mid \text { present })
$$

and can also be rewritten as 


$$
P(\text { future } \mid \text { present }, \text { past })=P(\text { future } \mid \text { present })
$$

making it a statement about what knowledge is required to adequately albeit probabilistically know the future of the system and any given present moment. From this point of view, ordinary differential equation based dynamical systems are Markov processes.

Both equations 1.3 and 1.4 can be made precise in measure theoretic terms and can be rewritten for any stochastic evolution on any kind of state space. In particular, when the state space is discrete and finite and the evolution discrete. It becomes a discrete time Markov chain (DTMC) whose evolution is uniquely determined by the transition matrix

$$
T_{i j}=P(j \mid i)
$$

where $P(j \mid i)$ is the probability of making a transition from state $i$ to state $j$ at any given discrete time instant.

One could generalize Markov chains to address the limitation of discreteness in time by looking at a continuous time discrete space analog of DTMC. The corresponding evolution equation then looks like

$$
\frac{d P_{i}(t)}{d t}=G_{i j} P_{i}(t)
$$

where $G_{i j}$ is called the generator of the continuous time Markov chain (CTMC) and is the replacement for the transition matrix $T_{i j}$ for DTMC. So, knowledge about the temporal evolution of the system $P_{i}(t)$ is a solution to the ordinary differential equation (2.2) and is known as the Kolmogorov Forwards Backwards 
Equation.

Following this strategy, one could remove the discreteness of the state space by going to a continuous state space. If one did this, one gets a fairly general stochastic process called the Markov process. Even if both time and state space are continuous, knowledge about the system still follows a probabilistic evolution equation. The generator $G_{i j}$ for CTMC in equation (2.2) is now a partial differential operator called the Fokker-Planck operator. The precise general form of the Fokker-Planck operator can get unwieldy but for simple state spaces like state spaces on real line $\mathbb{R}$ and line segment $[0,1]$, it is equivalent to the following partial differential equations (PDE) below. Both equations (1.7) and (2.5) are examples of PDE defined by Fokker-Planck operators.

$$
\frac{\partial c(x, t)}{\partial t}+v \frac{\partial c(x, t)}{\partial x}=D \frac{\partial^{2} c(x, t)}{\partial x^{2}}
$$

where $c(x, t)$ is the probability density of finding the system in state $x$ at time $t$. It should be noted that this partial differential equation is deterministic, even though the underlying system is stochastic.

When $v$ vanishes

$$
\frac{\partial c(x, t)}{\partial t}=D \frac{\partial^{2} c(x, t)}{\partial x^{2}}
$$

This equation is the classic diffusion equation originally constructed to model evolution of heat conduction in extended finite temperature materials and is identical to Schrodinger's equation. They also capture probabilistic evolution of particle in the absence of drift $v$.

Just as ordinary differential equations require some initial (boundary) conditions to make them completely and uniquely specified, PDE require boundary 
conditions. Taken together, the equation encodes all the knowledge one can acquire about the system.

In classical mechanics [58], the Lagrangian $L(q, \dot{q})$ is defined as a function of $x$ and $\dot{x}$, the position and velocity of the system. In an equivalent Hamiltonian formulation, the Hamiltonian $H(p, q)$ is defined as a function of $x$ and $q$, the position and momentum of the system. Any observable quantity of importance can be gotten as functions of the original parameters or via equivalent canonical transformations. The important feature is that the state evolution in these systems satisfies Euler-Lagrange family of PDE.

In quantum mechanics, the Euler-Lagrange PDE is replaced by the Schrodinger equation where the evolution of wave function is determined by the Hamiltonian operator. Once we solve the equation for a specific Hamiltonian operator, the wave function can be used to calculate the expectation value of any observable of interest. The Hamiltonian is the generator of probabilistic evolution, just like $G_{i j}$ from equation (2.2).

In SDS, the Fokker-Planck operator (generator of the Markov process) is the analog of Euler-Lagrange PDE operator and the Hamiltonian operator. Just as all relevant observables in classical mechanics satisfy this equation, all relevant observables in SDS satisfy a PDE based on the Fokker-Planck operator ${ }^{5}$.

\subsubsection{First-exits of SDS}

An important observable in SDS and stochastic processes in general is first-exit time, the time it takes for the state of a system to cross a certain threshold. In higher dimensions, it makes sense to think of exit from a domain with domain

\footnotetext{
${ }^{5}$ Fokker-Planck operator and the Schrodinger operator are related to each other through analytical continuation of time into the complex domain.
} 
boundary. One can associate with first-exit, a probability distribution function for the first-exit time or first-passage time (FPT), a random variable in this system.

$$
u(x, T)=P\{\tau<T \mid \mathbf{x}(t=0)=x\}
$$

where $u(x, T)$ is the cumulative probability of a particle starting at $x$ to reach the boundary before time $T$. $\mathcal{D}$ is the domain and $B d(\mathcal{D})$, its boundary. The associated random variable is represented by $\tau$. $\mathcal{L}$ is the Fokker-Planck operator. The associated PDE for the cumulative probability is given by

$$
\begin{aligned}
& \frac{\partial u(\mathbf{x}, t, T)}{\partial t}+\mathcal{L} u(x, t, T)=0 \text { for } \quad x \in \mathcal{D}, t<T \\
& u(x, t, T)=1 \quad \text { for } \quad x \in B d(\mathcal{D}), t<T \\
& u(x, t, T)=0 \quad \text { for } \quad x \in B d(\mathcal{D})
\end{aligned}
$$

Finding solutions to this equation is hard even numerically as even straightforward techniques like finite element methods for solving PDE become hard in higher dimensions. For certain one dimensional problems, $u$ may be solved exactly. We discuss one such example in chapter 3.

Instead a simpler PDE for mean first-passage time can be derived. For one dimensional PDE with state space lying in an interval $[a, b]$, the MFPT $(t(x))$ satisfies a PDE

For one dimensional PDE with state space lying in an interval $[0,1]$, the MFPT $(E[\tau(x)])$ satisfies a PDE

$$
D \frac{\partial^{2} E[\tau(x)]}{\partial x^{2}}+v(x) \frac{\partial E[\tau(x)]}{\partial x}=-1
$$

and $E[\tau(a)]=E[\tau(b)]=0$. In other words, $E[\tau(x)]$ is the mean time to reach 


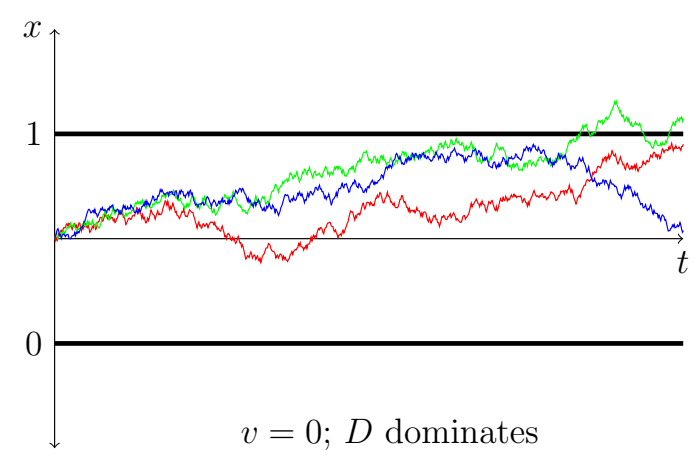

Figure 1.2: DDM

the boundary when starting at $x$. Equation (1.11) and it more general version is called the Pontryagin-Andronov-Witt equation. Higher order moments can be recursively derived from lower order moments. Except in few cases, these PDEs are all solved numerically when necessary.

While solving equation 1.11 and 4.5 gives MFPT and FPT in theory, they are the observed characteristics of the real system. What one needs instead is a way to find solutions to the inverse problem of determining $\mathcal{L}$ given $\hat{u}$. This issue is discussed more in chapter 4.

In Figures 1.2, 1.3, 1.4, and fig 1.4 we present some basic examples of typical paths of a SDS with constant diffusion coefficient and constant drift. They are all solutions of equation (1.1)The boundary points are 0 and 1. As the velocity (drift constant) increases, one can see that almost all paths exit boundary point 1. This is in contrast to the case where drift is zero.

First-exit problems and analysis surrounding them are useful ways of thinking about transient nature of stochastic processes and have found their use in the sciences and in engineering disciplines [105]. In many non-equilibrium systems, traditional equilibrium analysis washes away details about non-asymptotic dynamics and patterns. These finite time structures require finite time analysis of 


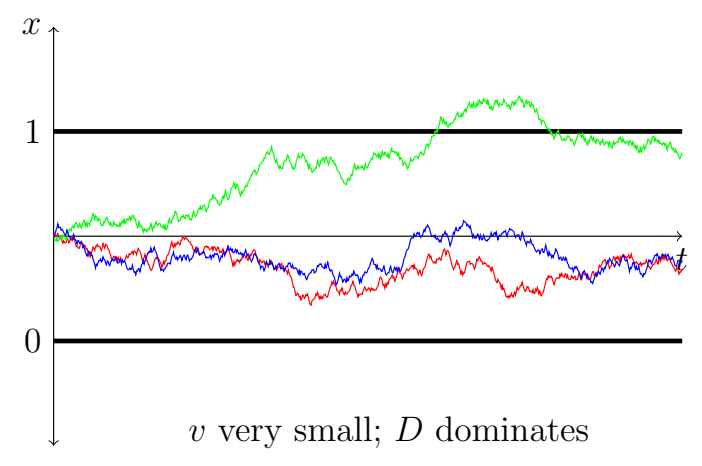

Figure 1.3: DDM

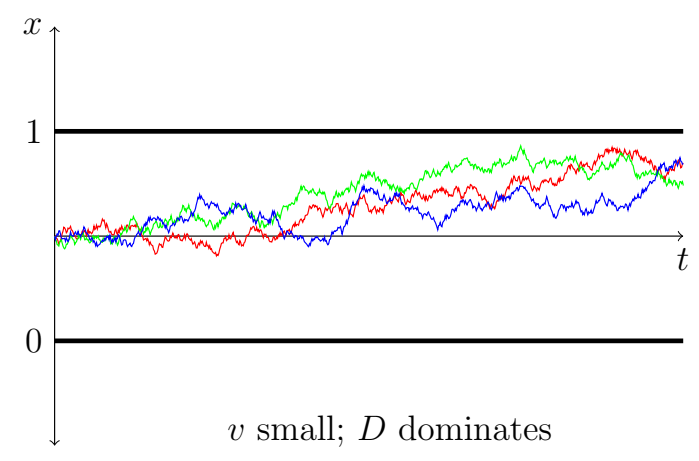

Figure 1.4: DDM

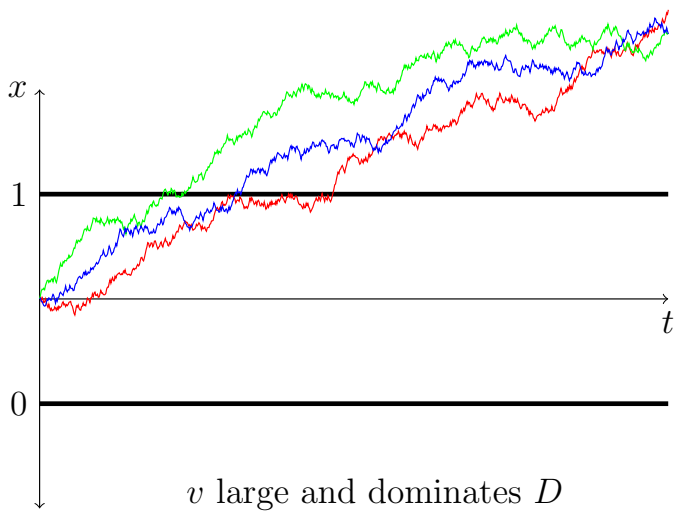

Figure 1.5: DDM 
such transients and FPT methods are one method to analyze such systems. In all these cases, the boundary and the domain is associated with some meaningful event for the system's evolution. In this dissertation, we identify transients and their timescales as ways to characterize biological reliability.

\subsubsection{From first-exits to reliability}

Previously, we borrowed the notion typically discussed in biological behavior, at the level of organisms, and used in to characterize aspects of physiological processes. As mentioned there, first-exit models of physiological processes at the nano and micro scale are effective models of those processes. As discussed earlier, we further characterize such processes as being reliable, a concept more natural in ethological settings.

Behavior of agents in environments is not just a time series; the state space evolution carries biological meaning. The time series and an interpretation of observable patterns carry semantic and pragmatic information. In other words, these patterns, codes and symbols carry biologically relevant semiotic information.

In general, one can identify the state variable $x$ with some biosemiotically salient behavior state, and imbue FPT and MFPT with biosemiotic salience. Using a reverse engineering perspective, one could ask whether it is possible to identify satisfactory parameters of an SDS so that the derived FPT or MPFT is close enough to observed FPT and MFPT respectively. For example, in the Venus flytrap example presented earlier, the trapping mechanism of the plant must op-

erate on a timescale that is considerably faster than the typical escape timescale of an insect capable of being trapped.

In our introduction to SDS, we introduced equation (4.5). The solution $u$ of the PDE subject to boundary conditions is a classical boundary value problem. From a 
reverse engineering perspective, one is interested in going in the opposite direction. Given $\hat{u}$, one is interested in determining the parameters that at least partially specify the Fokker-Planck operator $L$. In the language of this dissertation, one is seeking a subspace of space of all possible dynamical systems that satisfies the constraints set by $\hat{u}$.

Even when complete information about $\hat{u}$ is not available, a rough specification of this quantity like lower and upper bounds on probability, in the form of inequality is sufficient. This process of fitting to a desired $\hat{u}$ or $\hat{t}$ can be cast as a constraint satisfaction problem. And in the process of satisficing constraints, the reduction of the space of possible parameters of the SDS to good enough values is what this work calls fine tuning. That is, the internal structure of the agent stays within a viable set in order to guarantee a certain kind of reliability. And since this process does not use any explicit control modules, good enough reliability without control is feasible if successful fine tuning occurs. Only in the case where $\hat{u}$ is involved where one could convincingly argue that the constraint satisfaction based fine tuning gives rise to reliability of the worst-case kind. By this, we mean constraint satisfaction of various features of the probability density function directly, not their average properties like mean and variance.

While the focus of the dissertation is on MFPT based approach to reliability, the full problem is not as intractable as it sounds. The inverse problem of importance to us is also of interest to both robotics and applied mathematics. In the case of robotics, quick reverse engineering of natural laws obeyed by the environment of an agent is critical for the design of control system for artificial agents $[13,114]$. In several areas of applied sciences, automated reconstruction of dynamical systems from data speed up development of effective models of the phenomena $[107,108,113]$. In many instances, the phenomena are described in 
terms of spatio-temporal time series where the objective is to discover nonlinear partial differential equations from available data. It is this latter body of work that is partially aligned with the objective of the fine tuning based constraint satisfaction approach.

Our inverse problem may be more tractable than the problems considered by the above literature because the narrow specification of equation (4.5) contains a lot of details. We know the structure of the PDE operator and boundary conditions. Already this restricts the space of search many fold. Another way in which our inverse problem is more tractable is how the data for the inverse problem is specified: they are specified in terms of constraints of $\hat{u}$ and the boundaries. We are not limited by availability of data as is typical in such data driven approaches. On the other hand, the precise boundary conditions and non-trivial topological properties of state spaces may make the construction of space of solutions harder to construct. A more detailed space of solutions via finite element methods might be more desirable. Still, the problem remains challenging. In typical scenarios, given difficulty in finding an algorithm that solves the inverse problem, one could use MFPT or other approximate substitutes. It is this measure that is used in applications discussed in chapters 2 and 3.

For example, in a system with boundaries at $a$ and $b$, the constraint $E[\tau(x)]>>$ $\tau_{r}$ guarantees dynamics reaches the boundary reliably faster than the timescale $\tau_{r}$. Similarly, the constraint $E[\tau(x)]<<\tau_{a}$ guarantees dynamics avoids the boundary reliably faster than the timescale $\tau_{a}$.

The next section (and in more detail in chapters 2 and 3) picks up from this particular mathematical conception of reliability and uses SDS to social and psychological processes. Precise correspondence between MFPT based reliability and features of the psychological and social phenomenon is made. While this 
dissertation makes no concrete application to animal behavior, a sketch of first steps on how fine tuning based constraint satisfaction works as an algorithmic scheme is provided in chapter 4.

\subsection{Micro-nano physiology: processes and models}

While equation (4.5) is very general, only a simple version of Pontryagin-AndronovWitt equation [115] (equation (1.11)), the PDE governing MFPT is provided, anticipating applications discussed in chapters 2 and 3. Just like the equation for FPT, the PDE for MFPT is a typical boundary value problem with appropriate boundary conditions. Solutions to both these PDEs depend upon not only the drift and diffusion parameters but also on the geometry and topology of the boundaries. We present two categories of phenomena: escape phenomena and transport phenomena. Escape phenomena are richer as the MFPT in those cases are a function of geometry, topology, drift and diffusion parameters. Transport phenomena are more simple but still rich enough to encompass a wide variety of DDM models from chapter 3. Both phenomena exhibit sharp timescales, indirectly indicating the lack of variance in MFPT.

Escape phenomena $[66,116]$ are pervasive in the highly stochastic sub-cellular environments. We focus on two different kinds: escape from sub-cellular compartments and escape to cell membranes. Escapes from sub-cellular compartments are good abstractions of how electrical and chemical localization is enabled and dis-

abled in sub-cellular regions. Escapes to cell membranes are good abstractions of how macromolecules created in the cell reach the cell membrane dock themselves onto moving surface channels. The SDS models of these phenomena are three dimensional but the defining equations are the same form as equation (1.1) 


$$
\begin{gathered}
d x(t)=v(x) d t+\sqrt{2 D} d w \\
D \frac{\partial^{2} E[\tau(x)]}{\partial x^{2}}+v(x) \frac{\partial E[\tau(x)]}{\partial x}=-1
\end{gathered}
$$

- $x$ state variables corresponding to the position of the macromolecule or ion

- $\mathcal{D}$ is the domain (the cell) and $B d(\mathcal{D})$, its boundary (cell membrane) with the domain modeled as a smooth sphere or ellipsoid

- The boundary $B d(\mathcal{D})$ has both reflecting and absorbing regions

- $v(x)$ drift

- $D$ diffusion

- $E[\tau(x)]$ MFPT to reach one of the several absorbing regions located on the cell membrane

The parameters that can be fine tuned are the density of absorbing regions and drift. Typically, the molecules have internal motors that power their drift. Fine tuning this Brownian motor's capacity is one way to achieve a desired MFPT. Another parameter is the surface diffusive and density characteristics of docking proteins on the cell membrane.

Escapes from confined sub-cellular compartments, like the ones discussed earlier are important structural features that enable localization. These compartments provide mechanical ways to modulate chemical reactions within the cell. Such a localization also modulates release of macromolecules produced within the nuclear membrane. The models of these transport processes differ only in the geometry and topology of the domain. 
- $x$ state variables corresponding to the position of the macromolecule or ion

- $\mathcal{D}$ is the domain (the cell) and $B d(\mathcal{D})$, its boundary (cell membrane) with the domain modeled as a non-trivial geometric object.

- The boundary $B d(\mathcal{D})$ is mostly reflective with a few absorbing barriers

- $v(x)$ drift

- $D$ diffusion

- $E[\tau(x)]$ MFPT to reach one of the absorbing regions allowing the molecules to be released out of the compartment

Of predominant importance is the precise geometric shape and topological characteristics of the confined space as this alone strongly modulates the MFPT. Typically, extra-cellular chemical forces fine tune the geometric characteristics of the compartment.

Transport phenomena $[21,79,29]$ occur along various sub-cellular structures like actin filaments and cytoplasmic structures. They are responsible for transporting molecules to distant regions of large cells like neurons or transporting molecules in and out of the cell and between various sub-cellular organelles. Unlike the previous category of phenomena, the transporter molecules cling to skeletal structures, making the transport effectively one dimensional. This makes these models closest to the models used in chapters 2 and 3.

$$
\begin{gathered}
d x(t)=v(x) d t+\sqrt{2 D} d w \\
D \frac{\partial^{2} E[\tau(x)]}{\partial x^{2}}+v(x) \frac{\partial E[\tau(x)]}{\partial x}=-1
\end{gathered}
$$


- $x$ state variable corresponding to the position of the macromolecules on the sub-cellular transportation line

- $\mathcal{D}$ is the time constant

- The boundary $B d(\mathcal{D})$ are boundary points typically corresponding to the end points of the transport process

- $v(x)$ drift may be deterministic or stochastic

- $D$ diffusion may also change along the line

- $E[\tau(x)]$ MFPT to reach one of the several absorbing regions located on the cell membrane

In most intra-cellular transport processes, the drift exhibits non-trivial properties and corresponds to the velocity of the transporter molecular. These transporter molecules are modeled as a Brownian motor, a system capable of using stored internal energy to power itself. It is the characteristics of such motors that are fine tuned to achieve the desired MFPT.

The next section (and in more detail in chapters 2 and 3) picks up from this particular mathematical conception of reliability and uses SDS to social and psychological processes. Precise correspondence between MFPT based reliability and features of the psychological and social phenomenon is made. While this dissertation makes no concrete application to animal behavior, a sketch of first steps on how fine tuning based constraint satisfaction works as an algorithmic scheme is provided in chapter 4 . 


\subsection{Applications}

In mathematical sociology (Chapter 2), the work revisits older literature on Markov models of occupational mobility and generalizes it to show how SDS can model intra-generational mobility and escape from poverty traps. By looking at exittimes and exit-probabilities of escape from such traps, it points out features of social dynamics, such as transients, which are often missed by equilibrium macroeconomic and macro-sociological models.

In mathematical psychology (Chapter 3), the work looks at literature on driftdiffusion models (DDMs) of time constrained judgment and decision making. Inspired by models of escape from attractor and stochastic switching dynamics in simple neurophysiological processes, it proposes extensions of DDM, again illustrating the role of exit-times and exit-probabilities. These models could serve as reference models for experiments in cognitive psychology and motivate new experiments. That is, theoretical models with exact properties may be useful in comparing data from experiments.

While use of stochastic process models is not new in mathematical sociology or in mathematical psychology, the semantics of reliability, fine tuning and constraint satisfaction provide additional insight in the respective disciplines (chapters 2 and 3), suggest links among related fields within the same discipline, and generate insights and directions (chapter 4) for further exploration.

In both applications, the form of the models are the same. They are all onedimensional models with drift and diffusion term with dynamics that take place in an interval.

$$
d x(t)=v(x) d t+\sqrt{2 D} d w
$$




$$
D \frac{\partial^{2} E[\tau(x)]}{\partial x^{2}}+v(x) \frac{\partial E[\tau(x)]}{\partial x}=-1
$$

- $x$ state variables

- $[a, b]$ boundaries

- $v(x)$ drift

- $D$ diffusion

- $E[\tau(x)] \mathrm{MFPT}$

In the two applications, a different interpretation of constraint satisfaction and fine tuning is provided. The differences are due to the different endowed semantics.

\subsection{Summary}

The specific systems approach to understanding reliable behavior arose from attempts to generalize physiology and lift it to the systems level. In particular, by noting the surprisingly deterministic behavior of physiological processes at the nano and micro scale, and by considering this behavior in the context also of physiological processes at higher scales, the concept of reliable behavior is generalized. In doing so, models of nano and micro physiology are also lifted to the systems level. Once at this level, these models of reliable behavior are ready for application in any discipline. In the next two chapters, these models are used to model social and psychological processes, and offer novel insights.

By virtue of this lifting, additional insights not available to inquiry with a strictly disciplinary focus comes into view (Chapter 4). Along with such insights, questions regarding worst-case reliability and associated inverse problems point 
to gaps in the mathematical literature (Chapter 4). This gives rise to potentially fruitful areas of mathematical research. This, along with other virtues, make systems approach an integral part of scientific inquiry.

In system science,

- identifies FPT measures like MFPT with measures of resilience

- examines the role of physiology in the biology-centered perspective

- identifies a fine tuning based constraint satisfaction model that can -under some circumstances- replace control modules

In Sociology,

- introduces diffusion models to the theoretical literature on intragenerational mobility and poverty traps and proposes new ecometric measures for sociologists

In Psychology,

- introduces new DDM of judgment and decision making for modeling bias and uncertainty and connects DDM literature and ecological rationality literature 


\section{Revisting Markov models of Intragenerational Mobility}

\subsection{Introduction}

This chapter

1. proposes drift-diffusion models as models of intragenerational economic mobility

2. proposes drift-diffusion models in a finite interval as a model of poverty trap

3. identifies diffusion parameter as an ecometric measure - a family of measures important for urban sociologists

In Chapter 1, we introduced the idea of constraint satisfaction and fine tuning as a way to quantify certain kinds of reliability in systems. Mean first passage time (MFPT) is one such measure. In this chapter, we use MFPT based analysis to discuss models of poverty traps. MFPT in these models are shown to depend on the parameters of the stochastic dynamical system -drift-diffusion model (DDM)and their boundary conditions. By changing the drift $v$ and the diffusion $D$ of these SDS, one can change the MFPT, tuning the system to satisfy constraints specified in terms of exit time characteristics. We show that the language of fine tuning and constraint satisfaction introduced in Chapter 1 can be naturally cast as unexpected social policy levers -instruments that can help influence social conditions. Chapter 3 uses the same ideas to develop new models of dynamic 
decision making. And just like in this application, we point out insights generated by MFPT based notion of reliability.

In principle, the models of this chapter can be motivated independently of the systems philosophical considerations that motivated the dissertation; similarly, the conclusions we draw at the end of the chapter can be reached without explicit attention to the dissertation's systems orientation. However, the specific insights offered here arose out of the transdisciplinary nature of systems science reasoning and illustrate the pragmatic role of systems isomorphisms: they are scaffolds which help build bridges within disparate areas of disciplines; and connect distant disciplines that would otherwise not be connected; once connected, such scaffolds may be removed.

Specifically, the systems isomorphism from the previous chapter identifies continuous state space continuous-time Markov models as a candidate model for occupational and intragenerational social and economic mobility. The boundary conditions of the DDM further help model poverty traps. The use of DDM for intragenerational mobility, the proposal to identify the diffusion constant $D$ as an ecometric measure, and use of DDM with boundary conditions to model poverty traps are this chapter's main contributions.

In this chapter, we revisit an old idea from mathematical sociology of using Markov models to model social and occupational mobility $[8,14]$ with the intention of using the models to illuminate the role of environment. The models presented here are generalizations of past work previously used in that the dynamics take on values in continuous state space and evolve in continuous time. The original models used such models for both deductive and inductive tasks. However, the goal of this chapter is to reintroduce these ideas into the literature and illustrate their use in understanding issues of contemporary import, emphasizing their po- 
tential in simulating related social processes: economic mobility leading to escape from poverty; and loss of ability to a poverty trap.

Increasing economic inequality and lack of social mobility has become one of the few unifying issues among otherwise polarized public spheres of the Global North. Starting with the work of Piketty [98], quantitative social scientists have been increasingly drawn towards inequality, its origins and its subsequent durability, approaching them from different perspectives. Some models have sought to address and acknowledge more explicitly, the role of race, ethnicity, gender and even geography as determinants of economic and other life outcomes [31, 34, 100, 111]. Researchers have also focused on 20th century capitalist economic system in sustaining multi-generational poverty.

Such intense interests in these issues have attracted scrutiny of neo-classical economic models and their failure to incorporate sociological processes and mechanisms. These limitations have been addressed by recent work, both in public microeconomics, in the work of Chetty and his colleagues [32, 33]; and in sociology, in the work of Sampson and others [109, 62, 110]. Interestingly, both these literatures give prominence to the role of environment. A desire to complement the empirical approach of these bodies of work is one of the motivations behind this work discussed in this chapter. Further analysis of these issues may require completely new experimental and observational study designs but in lieu of data, computational social science models inspired by analytical sociology may help us gain insights through adequately designed analytical and simulation models.

This work, with a greater focus on individual social processes involving interaction between agents and their environment, is a contribution to such an effort. Typically, the machinery of stochastic process modeling -the solution of Fokker-Planck equation and numerical solution methods of stochastic differential 
equations- assume the state space of the system to be the real line. Furthermore, they are used as black-box models, and are not intended to encode detailed substantive mechanisms about the system. The models presented here are meaningful in that every free parameter and boundary conditions carry a sociological meaning. This distinguishes our models from the epistemological orientation of the models available in the literature.

In what follows, we motivate and develop models of intragenerational mobility by building on and adapting old models of occupational transitions and we demonstrate how they could be used to model the emergence of inter-individual variations and the role of environmental noise as a source of inequality. The models developed in this chapter, as extensions and generalizations of older occupational mobility models, turn out to be diffusion process models. Despite their pervasive use in other disciplines, these diffusion models have not been used as models of social processes.These simple diffusion models are known to produce rich long term dynamics from simple interaction mechanisms. The use of DDM as a generative mechanism oriented model within analytical sociology for use in computational social science is not contribution of this chapter.

In contrast to the coarse grained, equilibrium assumptions-based approaches of traditional macroeconomics, we focus on shorter term transients in wealth and other social demographic factors. Such transients are more important to model the volatility of day to day economic activity of individual social actors living well below the poverty line. While wealth and income better understood, the environmental origins of residual stochasticity are not emphasized in the literature. Also our model focuses on both the transient and stochastic nature of escape from poverty dynamics. 


\subsection{Social Mobility: Literature and Models}

Within the social sciences, the fields of sociology and economics have had a long tradition of research on social [91] and economic [40] inequality and mobility. Depending on specific traditions, one could either explain mobility using inequality as an explanatory variable or vice-versa. Within sociology [91], mobility is viewed broadly as either transitions between occupations or as attainment of status. While in economics [91], mobility is studied from a longer term perspective with more emphasis on a microeconomic approach based on human capital models which in turn are based on rational choice models [91]. In both disciplines, the emphasis has been more on intergenerational mobility with some discussion as to how one could adapt such models to study intragenerational mobility. Compared to the models proposed in this chapter, these are all discrete-time discrete-state space Markov models.

In recent years, the predominant mode of analysis has shifted towards descriptive and empirical analysis with very little theoretical model building. This move away from theory driven model building is much needed because of the growing gap between theoretical assumptions and ground realities seen in economic systems. While such a shift is correct, this approach has limitations because of a lack of data, and lack of insights on the underlying social mechanisms. Despite this, research questions and discussions allude to causal social processes and mecha-

nisms. In recent years, the advent of scalable methods [95] including machine learning have enabled researchers to detect patterns among dozens of variables with growing emphasis on causal inference. Still the emphasis remains on description and prediction [76]. A better approach, making use of theoretical and computational models, may be able to fill the lacuna and provide partial answers 
and counter-examples to questions and claims established using only inferential and descriptive methods. However, with a focus on prediction and the use of 'just-so' or 'as-if' models ${ }^{1}$, computational social science(CSS) methods lack the culture of aspiring for clear social mechanism-based explanations $[64,76]$. Analytical sociology $(\mathrm{AS})$, with its emphasis on mechanisms to explain macro-sociological phenomena, can resolve current limitations of computational social science by requiring computational models to be based on plausible models of underlying social mechanisms.

Before its current empirical turn, sociology had a distinct literature of theoretically motivated mathematical models [8, 41]. Unlike economics' emphasis on optimality and rationality based models, these models were stochastic dynamical systems based. One of the criticisms of these models were that they were overly simplistic and hence badly misspecified. However, in the intervening period, social science has adopted inferential approaches like event-history analysis, counting process models and other latent process models, all of which are closely related to the Markov models introduced here. Although the current work borrows heavily from models used in the physical sciences, the way we use them to model social processes has its intellectual lineage in the models of Boudon, Bartholomew and others $[8,14]$. The models of this chapter are continuous state space Markov models, in contrast to their discrete-time and discrete space Markov models of the

\footnotetext{
${ }^{1}$ Two categories of models from two different disciplines use this approach. One is the econophysics approach where social processes and social systems are treated as statistical mechanical systems. The literature uses models that are well-known in the equilibrium statistical physics literature. Similarly, inspired by economics, several game theoretical models are used as models of social interactions. In the former instance, the use of models of equilibrium ensembles for non-equilibrium social and economic processes are never justified; the starting premise is that complex social systems are just like equilibrium matter at finite temperatures. Similarly, even if rational actor hypothesis is not justified, the economists still insist on using game theoretic rationality as a model of economic agents; the claim is that the agents behave 'as if' they are rational actors.
} 
older literature.

This work focuses on intragenerational economic mobility [8, 14]. In older literature, given the difficulty of collecting data, upward movement of an individual in an organization via promotions, were used as a surrogate for more traditional wealth growth measurements, which are much more common in the economic literature. This upward movement can be transitions within the same organization or jumps to different jobs in a labor market. This can be contrasted with simple wealth and wealth growth as a measure of economic and social mobility of individuals over their lifespan. Occupational mobility data are fit to Markov models. Unlike the discrete transitions of the stochastic models used in sociology, we focus on a continuous state that evolves with time: a diffusion model. While partial differential equations have been used in the economics literature and more rarely in sociology, to the best of our knowledge, the use of diffusions to model social mobility seems to be novel.

\subsection{Models of Intragenerational Mobility}

Stochastic models of social processes were introduced in the early works of Coleman, Boudon, Bartholomew and others [42]. Several models were concerned about representing stochastic change over time, especially of intergenerational social and economic mobility, occupational mobility and social and economic mobility over an individual's lifecourse $[8,14]$.

A Markov chain is a discrete dynamical system that evolves stochastically. Suppose the state space of the model is indexed by natural numbers $\{1,2, \ldots N\}$. The evolution of this dynamical system is given by a transition matrix $T_{i j}$ where the entries of this matrix correspond to conditional probabilities of transition from 
one state $i$ to another state $j$. That is,

$$
T_{i j}=P(j \mid i)
$$

So the probility of finding the system in a given state after $n$ time steps can be extracted from the matrix $T^{n}$. In the continuous time case, one can show that $P_{i}(t)$, the probability of finding the system in a state $i$ at time $t$ is given by

$$
\frac{d P_{i}(t)}{d t}=G_{i j} P_{i}(t)
$$

where $G_{i j}$ is called the generator of the CTMC.

The models [8] discussed in this literature are all variations of Markov chains, Discrete-time Markov chains(DTMC), with discrete time steps and discrete statespaces; continuous-time Markov chains(CTMC), with continuous-time and discrete spaces; time-inhomogenous CTMC, with temporal heterogeneity in the transitions; and stratified CTMC, with multiple Markov chains for different demographic sub-populations.

In these models, the state space is an ordered space corresponding to rising social status of the occupations. The mobility dynamics stops when the individual drops out of the work force due to exogenous factors. So, given data on occupational transitions, one can infer the generator $G$ or $T$.

Alternatively, one can model associated upward changes in status of wealth coming from upward mobility in occupation. Suppose we wish to further stratify the social actors by various social and demographic variables. One straightforward approach is to vary the drift and diffusion parameters accordingly. These model parameters can be used to denote external environmental conditions or intrinsic individual, social or other demographic states. We discuss this in the later sections 
of this chapter.

The continuous time continuous state Markov chain is what is of interest in this chapter, where the state variable is an individual's wealth $x(t)$. We assume that there is a steady growth in wealth $v$ and environmental fluctuations $D$ that randomly perturb this steady growth of wealth. Taken together, the dynamics are captured by the following stochastic differential equation.

$$
d x(t)=v d t+\sqrt{2 D} d w
$$

One can show that the solution of the stochastic dynamical system satisfies the diffusion equation with drift $v$ and diffusion constant $D$

$$
\frac{\partial c(x, t)}{\partial t}+v \frac{\partial c(x, t)}{\partial x}=D \frac{\partial^{2} c(x, t)}{\partial x^{2}}
$$

or when drift $v$ vanishes

$$
\frac{\partial c(x, t)}{\partial t}=D \frac{\partial^{2} c(x, t)}{\partial x^{2}}
$$

$c(x, t)$ is the probability density of finding an individual with wealth $x$ at time $t$. The important point to chapter is that solutions to partial differential equations make sense only after the appropriate boundary conditions and initial conditions are defined. The solution to the above equation when $\mathrm{x}(0)=0$ and the range of $x$ is $\{-\infty, \infty\}$ can be shown to be

$$
c(x, t)=\frac{1}{\sqrt{4 \pi D t}} e^{-x^{2} / 4 D t}
$$

Similarly, the solution to equation (2.4) can be shown to be 


$$
c(x, t)=\frac{1}{\sqrt{4 \pi D t}} e^{-(x-v t)^{2} / 4 D t}
$$

And the solution to equation (2.5) under absorbing boundary conditions can be shown to be

$$
c(x, s)=\frac{\sinh \left(\sqrt{\frac{s}{D}} x_{<}\right) \sinh \left(\sqrt{\frac{s}{D}}\left(L-x_{>}\right)\right)}{\sqrt{t D} \sinh \left(\sqrt{\frac{s}{D}} L\right)}
$$

In all cases, the solution is represented in terms of the Laplace transform $c(x, s)$ of the original solution $c(x, t)$ as the transformed solution can be exactly calculated. $L$ is the length of the interval and $x_{<}=\min \left(x, x_{0}\right)$ and $x_{>}=\max \left(x, x_{0}\right)$ where $x_{0}$ is the initial starting condition. Since solutions to equations along the entire dimension, (2.5) and (2.4) are Gaussian functions with time dependent mean and variance, time evolution of the probability density function can be interpreted easily. The analog equation (2.8) where drift is non-zero is given by

$$
c(x, s)=\frac{e^{v\left(x-x_{0}\right) / 2 D}}{D w \sinh w L} \sinh \left(w x_{<}\right) \sinh \left[w\left(L-x_{>}\right)\right]
$$

where $w=\sqrt{v^{2}+4 D s} / 2 D$

In Figure (2.1), we plot the time evolution of equation (2.5) corresponding to diffusion in one dimension. At short times, the probability density function is localized at the initial starting point at $x=0$. As time increases, the particle diffuses, the increased variance denotes the delocalized nature of the particle but the peak probability density stays at the same point, consistent with $v=0$.

In Figure (2.2), we plot the time evolution of equation (2.4) corresponding to diffusion in one dimension with positive drift. At short times, the probability density function is localized at the initial starting point at $x=0$. As time increases, 
the particle diffuses, the increased variance denotes the delocalized nature of the particle but the peak probability density shifts towards the right, consistent with the positive velocity $v$ in that direction.

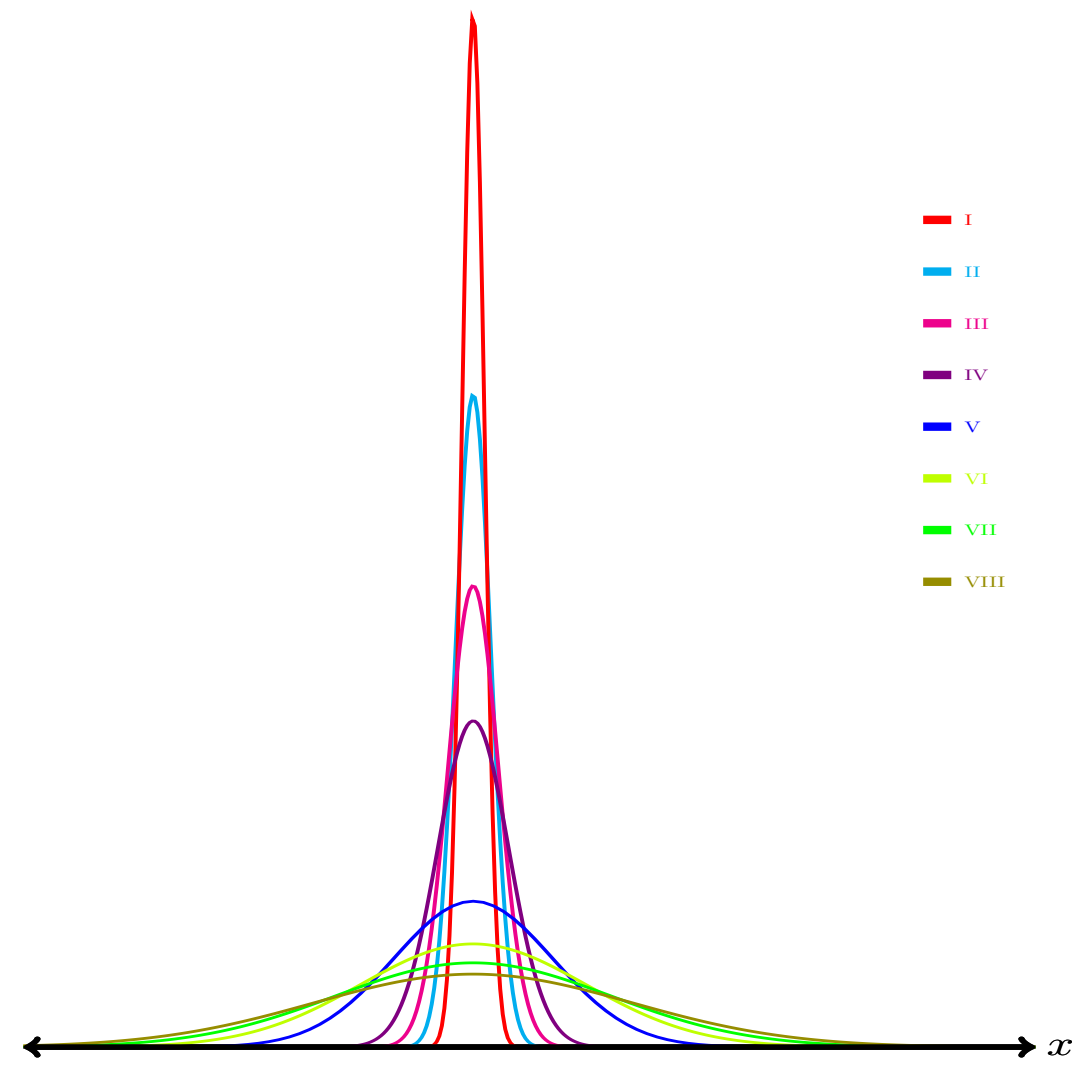

Figure 2.1: Diffusion on an infinite line

Roman numerals are numbered in the direction of increasing time. The $x$ axis is the state space and $y$ axis is the probability density.

In figure ( 2.3 ), we plot the first passage time density for a particle starting at the center of a finite interval to reach one of the boundaries. As the diffusion increases, in the absence of drift in either direction, the time to reach the boundary becomes shorter. Also important is the sharpness of the peaks in FPT density. We discuss variations of FPT under various initial conditions in chapter 3 where 


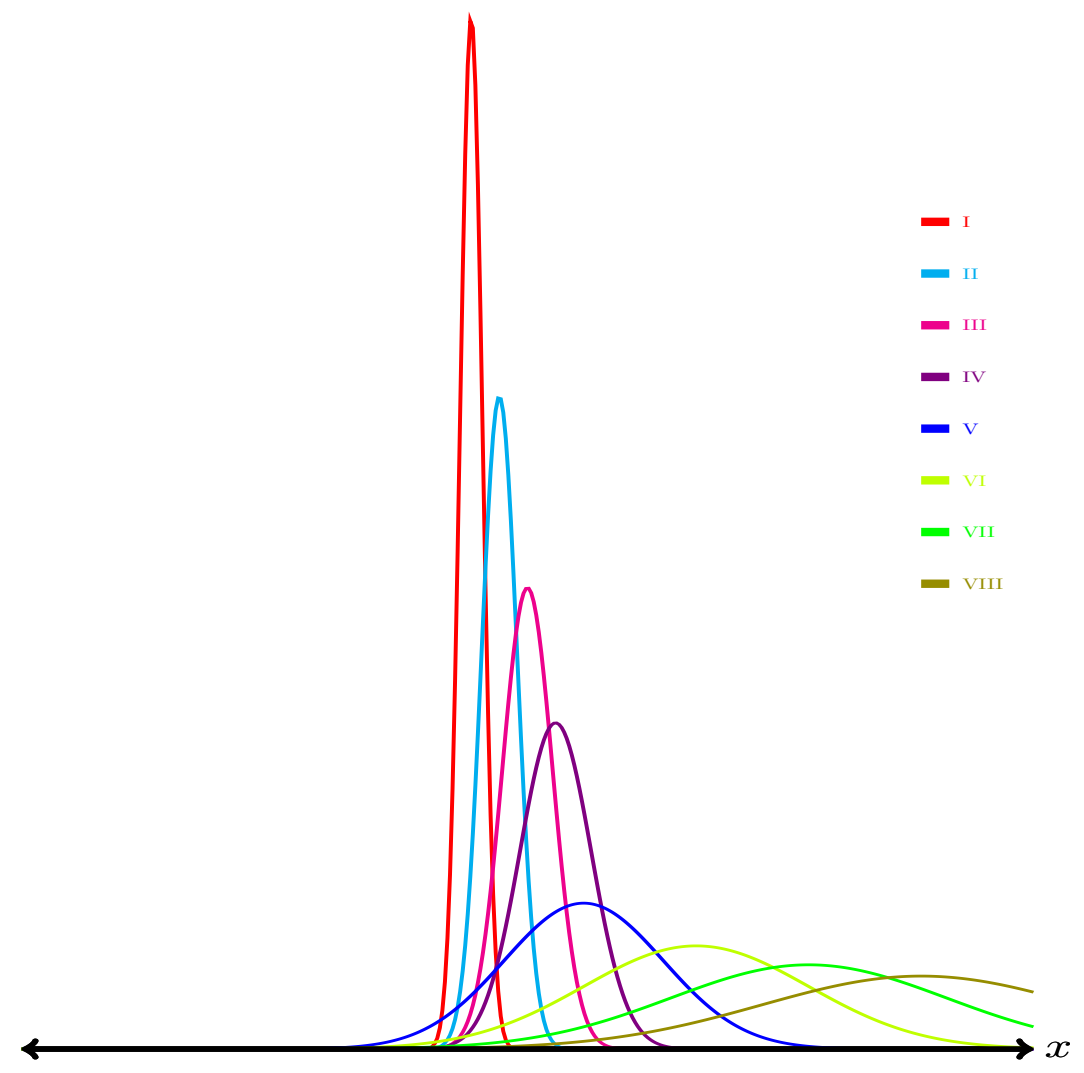

Figure 2.2: Diffusion with drift on an infinite line

Roman numerals are numbered in the direction of increasing time. The $x$ axis is the state space and $y$ axis is the probability density.

the context of response time analysis in cognitive psychology.

Next we discuss models of escape from poverty by analyzing mean first passage time (MFPT), the key recurring concept used to characterize reliability as defined in chapter 1. Both in micro nano physiology, and in other physical and applied sciences, MFPT analysis is used for studying exit phenomena $[105,115]^{2}$. The key

\footnotetext{
${ }^{2}$ Models of FPT were discussed in the physical sciences as models of chemical reactions and diffusion in media. Simultaneously, as mentioned in the first chapter, the models were also used in engineering as models of dynamic noise in several areas. As the field of molecular biology and sub-cellular biology expanded its ability to attain precise measurements of diffusion constants in sub-cellular domain, models of macro bio-molecular movement began to be modeled as diffusion processes. Currently, almost all nano and micro physiological processes in highly stochastic
} 


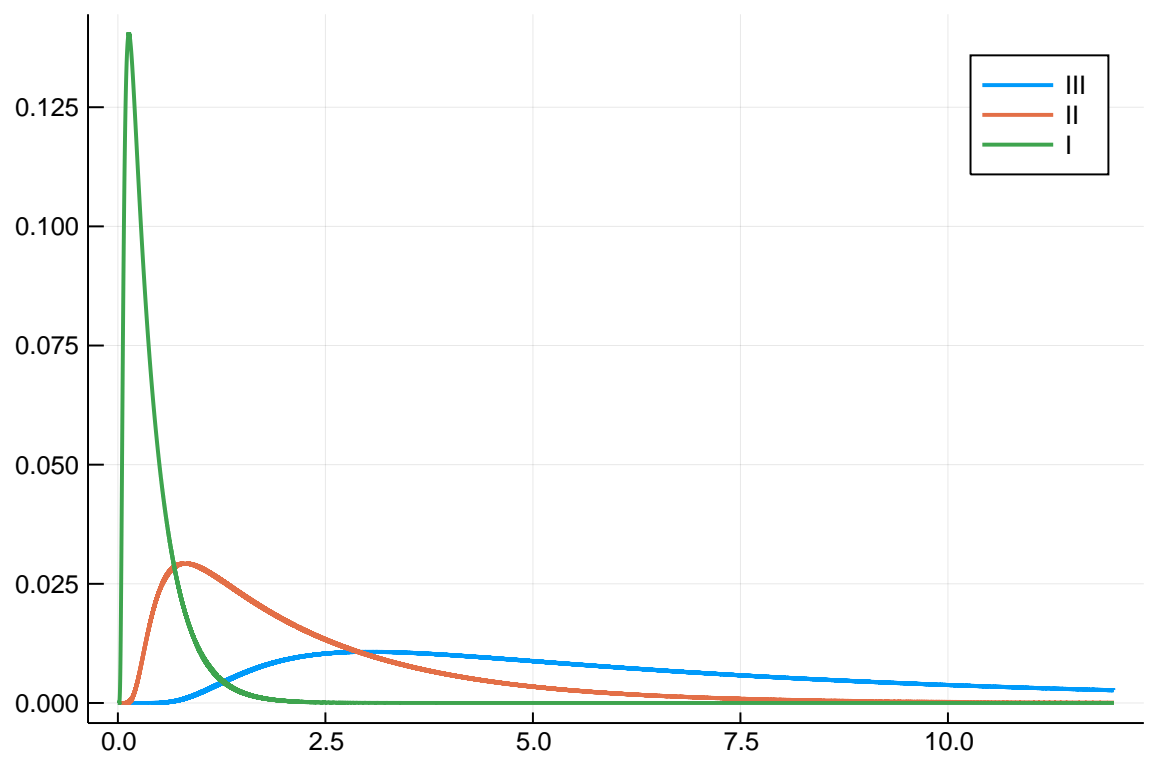

Figure 2.3: First passage time density for varying diffusion constants Roman numerals are numbered in the direction of increasing diffusion.

premise in this analysis is the idea that MFPT is an adequate measure of FPT as a whole. That is, FPT has peaks sharp enough for the phenomena to be predictable using only the first moment of FPT. Such an assumption is backed up by empircal literature $[66,54]$ in nano and micro physiology and in other disciplines.

In the next subsection, we discuss various MFPT curves that are analytically calculated as solutions of PDE. They are functions of the initial starting point, drift and diffusion terms and show peaks. This suffices to operationalize the fine tuning based constraint satisfaction mechanism introduced in the first chapter. In all these cases, the MFPT $t(x)$ of the one-dimensional drift-diffusion model corresponds to the mean time for a wealth to cross a certain threshold starting at a point $x$. Crossing the threshold from below corresponds to escaping the poverty trap.

Consider the case when there is no drift but the system has two absorbing environments are modeled as diffusions. 
boundary conditions. Figure (2.4) shows MFPT in such situations. By merely changing the diffusion constant $D$ and making sure that the initial starting point is within a certain range, we could guarantee that the system's MFPT is above a certain threshold, if such a constraint is needed for viability. The blue curve is such an example. In contrast, we could also guarantee that the MFPT is below a certain desired threshold, if such a constraint is needed for viability. The green and orange curves are examples. One could also desire an MFPT that is below a certain threshold but also above a certain other lower threshold. In that case, one could pick the orange curve as the one that satisfies the viability constraints. The associated range of diffusion are then the fine tuned parameters of this stochastic dynamic system.

\subsubsection{First-passage time models of escape from poverty}

In the literature, some notions of mobility involve the idea of status attainment. That is, mobility is measured by looking for attainment of a social or economic status. Some debates on inequalities are around the lack of growth of the middle class, focusing on intergenerational mobility through status attainment. Also, recent work in public microeconomics like Moving To Opportunity ${ }^{3}$ projects have tried to measure the effects of exposure to certain environments on the economic prospects of individuals over the course of their lives. Inspired by such research on status attainment and environmental exposure and other studies of urban poverty, a specific question of interest is the idea of time to escape from poverty or return to poverty. Next we introduce a model of one dimensional first-passage time on an interval model and use it to model escape from poverty.

In this and subsequent discussions, we focus on a simplified model of poverty

\footnotetext{
${ }^{3}$ https://www.huduser.gov/portal/datasets/mto.html
} 
dynamics. We assume that an actor escapes poverty permanently once the actor's wealth crosses a certain threshold. Such questions about stochastic systems are discussed under the rubric of first-passage time problems (FPT) [105, 101] $]^{4}$. That is, the distribution of times of the state variables of a system to cross a threshold and its associated moments. For simplicity, we assume that the wealth of an individual is always positive and the individual escapes poverty once the wealth crosses the threshold $L$. Condition $x=0$ corresponds to an actor's descent into permanent poverty. Poverty traps are then the region $[0, L[$ where actors have a chance of escaping poverty.

In the language of diffusion problems, $L$ is the absorbing point and the diffusions stops once it crosses the boundary. In these systems, the following natural quantities are of importance

- What is the first-passage probability $F(t)$ to the exit point?

- How long does it take for the particle to reach the exit point?

- What is the survival probability $S(t)$ ? The probability that a diffusing particle does not reach the absorbing boundaries.

- How do these quantities depend on the initial starting point?

- What is the mean exit time, that is, the on average time until the particle hits the boundary, starting at an initial time?

- What is the conditional exit time, that is, when does the particle reach one boundary (while avoiding the other $x=0$ )?

\footnotetext{
${ }^{4}$ Mathematical discussions in the rest of this article closely follows discussion in Redner, especially Chapter 2
} 
For what is to follow, we study models with or without $\operatorname{drift}(v)^{5}$ under various boundary conditions at $x=0$ and $x=L$. In some cases, we directly calculate the mean first-passage time (MFPT). A reflecting boundary condition is one where the particle simply bounces back. Transmission mode is one where there is reflection at the origin and absorption at $x=L$. The particle starts closer to the reflecting point.

\subsubsection{MFPT under different conditions}

Here we present results of first-passage time calculations under various boundary conditions of standard pure-diffusion or convection-diffusion systems in a bounded interval. We refer the reader to Redner's book [105]for more details. These results are not new, but their application to discuss poverty traps is.

\section{Diffusion with no drift in absorption mode}

$$
t(x)=\frac{1}{2 D} x_{0}(L-x)
$$

Figure (2.4) shows the quadratic variation of MFPT for case with pure diffusion. The survival probability can be shown to be

$$
S(t) \propto e^{-t / \tau_{1}} \text { with } \tau_{1}=L^{2} / D \pi^{2}
$$

\section{Diffusion with drift in absorption mode}

$$
t(x)=\frac{L^{2}}{2 D} \frac{1}{P e} \frac{\left(1-e^{-2 u P e}\right)-u\left(1-e^{-2 P e}\right)}{1-e^{-2 P e}}
$$

\footnotetext{
${ }^{5}$ The problem of $v \rightarrow 0$ and $v=0$ is qualitatively different and mathematically intricate. We merely mention here that both solutions have to be calculated independently.
} 


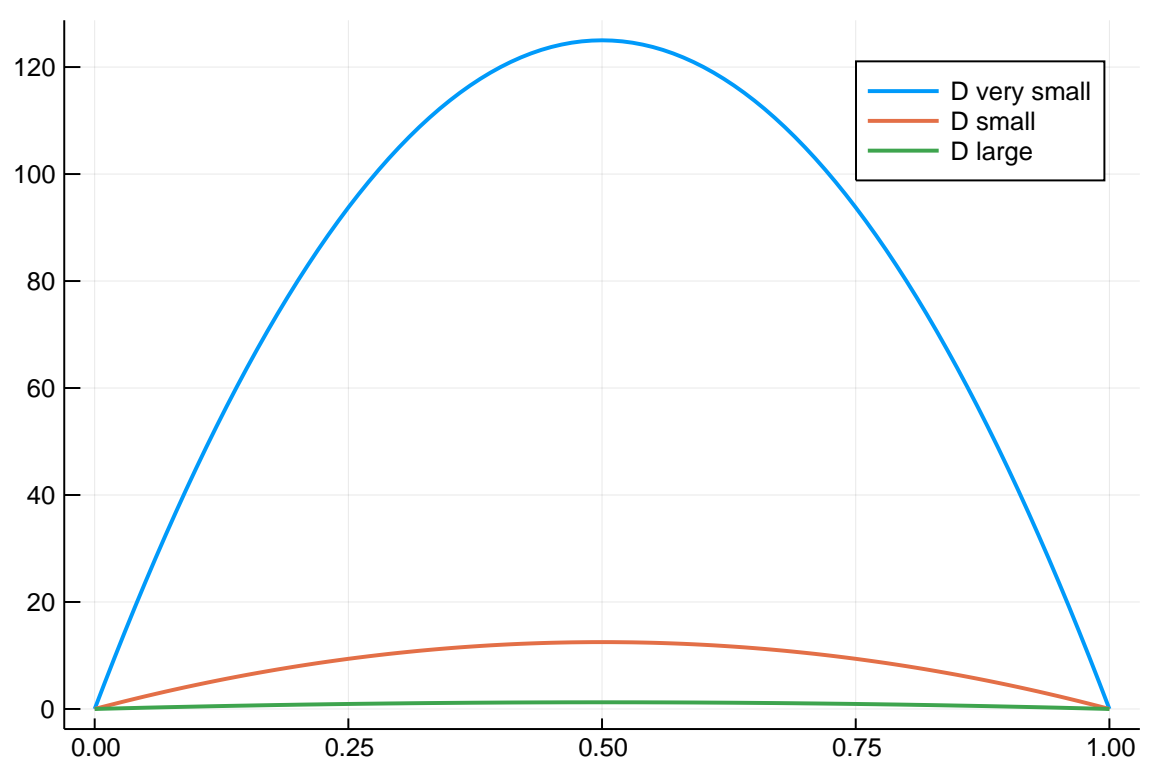

Figure 2.4: MFPT for diffusion on an interval

MFPT as a function of starting point $x$ is plotted for different diffusion constants based on equation (2.10). As the diffusion increases, the expected time to reach the boundary gets smaller.

where $u=x / L$ and $P e=v L / 2 D$ is called the Peclet number. The survival probability

$$
S(t)=e^{-t / \tau_{2}} \text { with } \tau_{2}=\frac{L^{2} / D \pi^{2}}{1+(v L / 2 \pi D)^{2}}
$$

Figure (2.5) plots MFPT for diffusion with drift scenarios. As the magnitude of drift increases, the expected time to reach the boundary reduces. Depending on the distance from the boundary, the relative time to get to the boundary depends on the sign of the drift.

\section{Conditioned first-passage problem with drift and absorbing boundaries}

To illustrate the nature of these calculations, we illustrate the MFPT calculation for a conditioned first-passage time problem. 


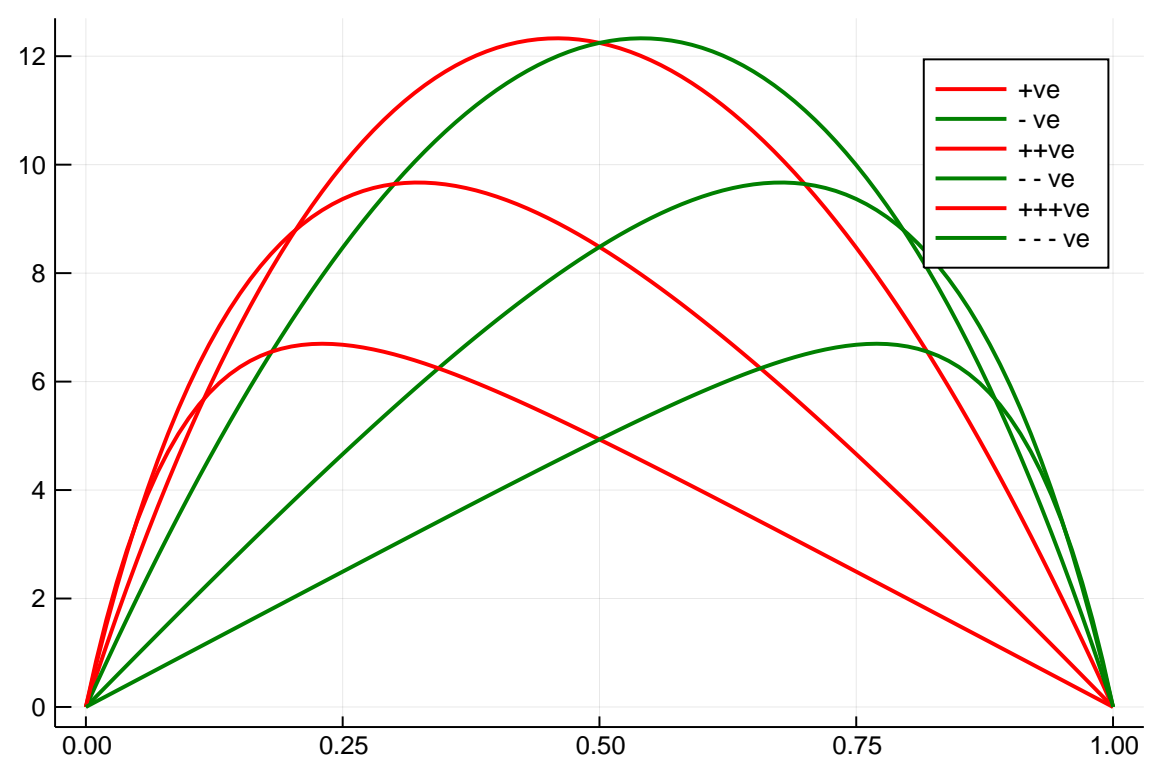

Figure 2.5: MFPT for diffusion on an interval

MFPT as a function of starting point $x$ is plotted for different drift velocities in opposite directions.

Eventual hitting probability to reach the absorbing boundary without reaching the origin is $E_{+}$

Eventual hitting probability to reach the origin without reaching the absorbing boundary is $E_{-}$

Mean conditional exit time of a particle to reach the absorbing boundary without reaching the origin is $t_{+}(x)$ Mean conditional exit time of a particle to reach the origin without reaching the absorbing boundary is $t_{-}(x)$ Boundary conditions $E_{+}(0)=0$ and $E_{-}(L)=1$ and vice versa for the opposite condition. Similarly, for $t(0)=t(L)=0$ as boundary conditions for $t(x)$

$$
\begin{gathered}
D \nabla^{2} t(x)+v(x) \cdot \vec{\nabla} t(x)=-1 \\
D \nabla^{2}\left[E_{ \pm}(x) t_{ \pm}(x)\right]+\vec{v} \cdot \vec{\nabla}\left[E_{ \pm}(x) t_{ \pm}(x)\right]=-E_{ \pm}(x)
\end{gathered}
$$




$$
D E_{ \pm}(x)^{\prime \prime}+v E_{ \pm}(x)^{\prime}=0
$$

$$
D\left[E_{ \pm}(x) t_{ \pm}(x)\right]^{\prime \prime}+v\left[E_{ \pm}(x) t_{ \pm}(x)\right]^{\prime}=-E_{ \pm}(x)
$$

$$
t_{+}=\frac{L}{v}\left(\frac{1+e^{-v L / D}}{1-e^{-v L / D}}\right)-\frac{x}{v}\left(\frac{1+e^{-v x / D}}{1-e^{-v x / D}}\right)
$$

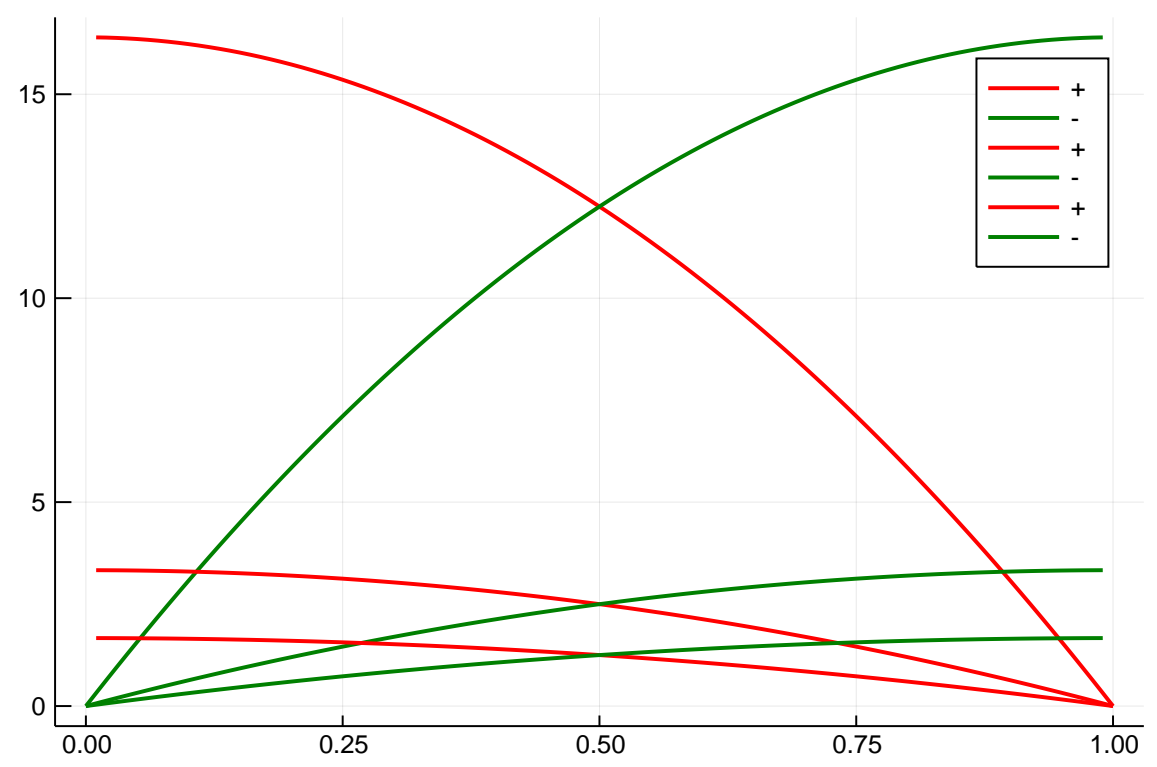

Figure 2.6: Conditional MFPT diffusion with drift on an interval MFPT as a function of starting point $x$ is plotted for different diffusions but keeping drift constant.

In figure (2.6), we note the differences in conditioned MPFT as we increase diffusion. As diffusion increases, the MFPT reduces overall. The red and the blue curves correspond to the boundary condition that is conditioned to reach one of the boundaries. 


\section{Diffusion with drift in transmission mode}

Mean exit time ${ }^{6}$ for a particle starting at the reflecting boundary is given by

$$
\langle t\rangle=\frac{L}{v}-\frac{D}{v^{2}}\left(1-e^{-v L / D}\right)
$$

\section{Diffusion with fluctuating drift}

Building on the above models, one can use stochasticity to any deterministic component of the model. More specifically, if we promote drift to a random variable that switches between a positive and negative values at a certain $\mathrm{rate}^{7}$, one can repeat the conditioned first-passage problem, now with a fluctuating $\mathrm{drift}^{8}$. We discuss this in the next chapter in greater detail.

\section{Absorbing boundaries along one direction and a transmission type boundary in another direction}

Consider a rectangular region with different diffusion and drift constants $D_{1}, v_{1}, L_{1}$ and $D_{2}, v_{2}, L_{2}$ along the $x$-axis and $y$-axis respectively. Since the two dynamics ${ }^{9}$ are independent, it is straightforward to discuss this in terms of time constants that were introduced above $\tau_{1}, \tau_{2}$ and $\langle t\rangle$. If $\langle t\rangle \gg \tau_{1}$, then on average, most of the paths are transmitted before it gets absorbed in the other direction. Similarly, if $\langle t\rangle \ll \tau_{1}$, then most of the paths are absorbed before they get transmitted. We will use this model to describe two dimensional escape from poverty in the next

\footnotetext{
${ }^{6}$ In this case, a particle is injected near the reflecting boundary at $t=0$

${ }^{7}$ This is a continuous-time Markov chain with two states.

${ }^{8}$ We will not sketch the mathematical details but provide heuristic argument on the consequences for the first-passage times. At very low fluctuation rates, the system behaves as if there were no fluctuations and the results mimic the one without randomness of the drift parameter. For very high fluctuation rate, the system essentially cancels the drift leaving a pure diffusion component.

${ }^{9}$ One could do any other combination of boundary conditions in a similar manner.
} 
sub-section.

\subsubsection{From one dimensional first-passage time problems to escape from poverty and intragenerational mobility}

Before we impart meaning to these models by mapping them onto social processes underlying wealth based intragenerational mobility, let us summarize the tools and exact results we've presented previously.

When we moved from simple DTMC to CTMC, we moved from a discrete-time transition matrix based probabilistic evolution to a first-order differential equation called the Kolmogorov forward-backward differential equation (2.2). Similarly, when we moved from CTMC to a diffusion process, the resulting evolution equations are Fokker-Planck type we discussed in the previous chapter. In doing so, the emphasis shifts from state space point of view to the probability density evolution equations of observables. Quantities like expected probability of exit from a given starting point $x, E(x)$ and the expected time to exit the interval starting from $x, t(x)$ all satisfy PDEs belonging to the Fokker-Planck type. The FPT probability distribution function (PDF) also satisfies a PDE but even in the case of one-dimensional FPT problems, it satisfies an infinite series. In higher dimensions, calculating the PDF requires sophisticated techniques. In this chapter and the next, we focus on MFPT.

First passage models are ideally suited to model phenomena where exit from or to an observable phase is stochastically dependent on time. The extant literature on first-passage phenomena all share this characteristic [105]. Exemplary phenomena from nano and micro physiology presented in the previous chapter all exhibit this characteristic. Another important feature that these are properties of individual state-space histories and are transient in nature. Both these features of 
these models provide clues about what kinds of social processes are best modeled as first-exit problems.

The field using the model class identical to ours is finance where Markov models of wealth and asset dynamics are common in financial modeling. Several aspects of financial contracts are modeled as first-passage time questions. There is also a burgeoning literature on heterogeneous agent models. We discuss this in the concluding section of this chapter and make important comparisons with our models.

In macroeconomics, wealth and income distributions are sometimes studied using stochastic differential equations $[10,9]$. In such works, the focus is on expected values of wealth, their distributions and skewness, and not in the specific nature of individual histories or the time to cross a threshold. This is because of the macroeconomic nature of their questions. We discuss extensions of the analysis of this chapter to such models in Chapter 4.

In contrast, macrosociologists are also interested in demographic and social determinants of wealth over the life-course $[17,44]$. Their models based on discrete event based data are more aligned with the focus of this chapter because of shared emphasis in modeling life-course trajectories as transients of stochastic systems. But our continuous state space approach is different than their approach based on continuous-time semi-Markov models. The measures of intensity of events and their mediating factors are of importance. Also our interest in using FPT models for computational and analytical purposes are in contrast to the statistical nature of their models.

Recently, a greater appreciation of the phenomena unique at the level(s) between macro and micro have come into focus. Researchers studying dynamics note high volatility $[36,6]$ in income and wealth of individuals below the poverty line. 
This is due to the fact that both income and expenses are often unpredictable below the poverty line. Also, both development economists [7] and sociologists of urban poverty $[111,109]$ argue for a multi-dimensional definition of poverty. That is, focusing only on wealth and income is of limited value and other sociological and social-psychological variables play important roles in understanding wealth stagnation or descent into poverty.

So far, none of the above research areas and their research questions really warrant a model having a finite extent domain and domain boundary. The key additional feature of a social phenomenon like intragenerational of life-course economic mobility, requiring a finite extent domain for the model, is the concept of poverty traps [15],which has been used to explain failures of well-known macroeconomic and microeconomic thinking about development, especially persistence of poverty.

In simple terms, poverty traps are sub-optimal equilibria of society viewed in economic terms. The efforts required for designers of markets to move it towards more optimal equilibria are very different than traditional economic policy discussions as they involve the study of systems that defy market idealizations ${ }^{10}$. There are three categories of explanations for why such traps arise and persist: critical thresholds of economic transformation exist; dysfunctional institutions and extra-market forces generate inertia that resist developmental processes; and neighborhood effects corresponding to effects of sociological variables arise in spatial settings.

While neighborhood effects can be modeled using variations in parameters of

\footnotetext{
${ }^{10}$ Conventional economics starts with the assumption that there is a market to begin with. However, economists studying the nature of traps point to conditions that are not at all marketlike. That is, the socio-economic conditions cannot justify market equilibrium assumptions. This and other observations made by developmental economists suggest that poverty traps have to be studied and modeled on its own; market microeconomics are not the right starting point.
} 
the stochastic dynamical system, the defining characteristic of the presence of critical thresholds is the most interesting because of its similarities to first-exit phenomena. Specifically, given the understanding of poverty traps in terms of failure of market forces, the threshold economic variables above which market forces begin working to produce efficient markets are natural candidates for domain boundaries ${ }^{11}$.

While empirical studies have focused on individual level variables: psychological, social-psychological, and other variables, and how they mediate economic outcomes, the social ecology of individuals and groups have been ignored until recently. Careful analysis of geographical variations in economic and social outcomes indicate systematic correlations in collections of variables with changes in neighborhood. Urban sociologists speculate that stable social processes, which are hard to study empirically, maybe the causal force behind persistence of various economic development measures. In order to incorporate such social mechanisms into generative and inferential models, surrogate measures have been proposed [109] and confirmed to be better predictive of economic and social development indices. These measures are called ecometric measures. Such measures include measures of organizational efficacy within residents of a block, neighborhood disorderiness, data on emergency and other social service calls, etc.

Generative models of poverty traps and ecometric measures have not been proposed in the literature. The drift-diffusion models discussed in this chapter are thus candidate models. Specifically, we identify the boundaries of the models with trap boundaries, and the diffusion parameter with an ecometric measure.

\footnotetext{
${ }^{11}$ For example, in neighborhoods with durable poverty, most credit and debt facilities are informal and based on illegal and unlawful lending agents. This informal market exists because the formal legal banking and financial institutions provide financial instruments for debt amelioration only if individuals satisfy certain economic criteria. In such cases, it can be argued that the market has failed -in the economic sense- to cater to the needs of the poor.
} 
This model of poverty trap with ecometric parameters is a novel contribution to urban sociology.

An improved model of poverty traps is in terms of escape from attractor scenarios $^{12}$. Instead, we make simplifying assumptions and treat the dynamics in terms of simple constant drift-diffusion models. In the future, we plan to create models without these simplifying assumptions. Associated neighborhood effects can be modeled in terms of neighborhood dependent drift-diffusion parameters. We discuss this and other extensions in Chapter 4.

Although all MFPT equations presented previously could be used as models of social mobility in the presence of traps, we focus on models based on (2.13) which we use in the next section.

\subsubsection{Modeling mobility dynamics and escape from poverty traps}

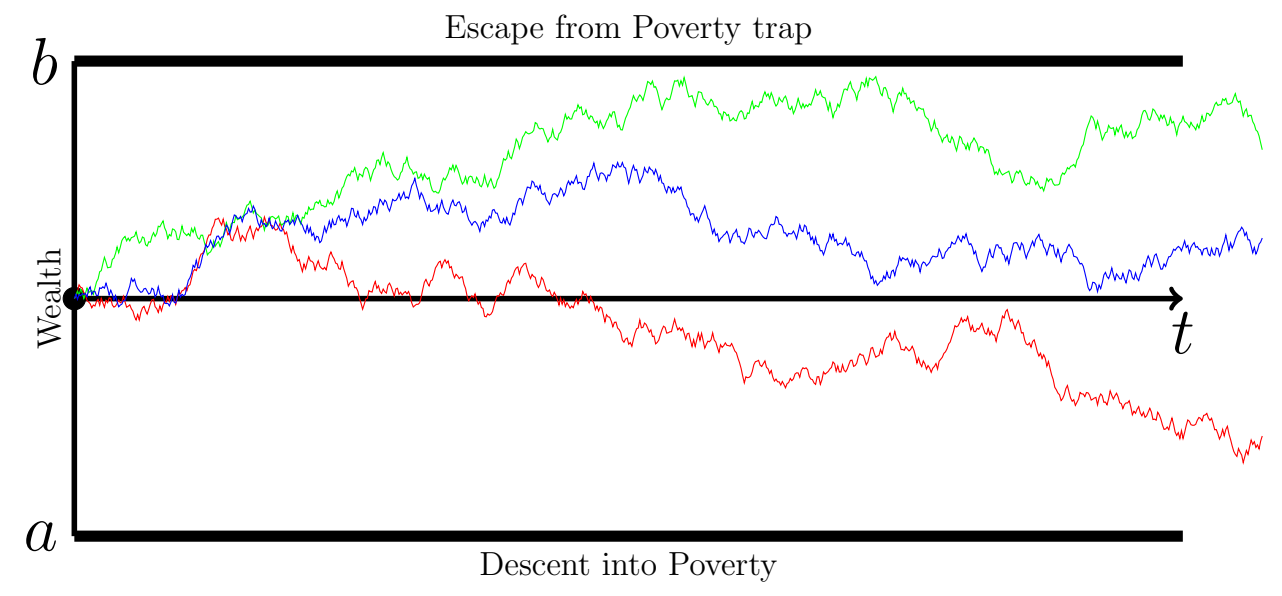

Figure 2.7: Model of poverty traps

Having presented a series of diffusion models, we are now ready to use FPT analysis to model social and economic mobility dynamics. We discuss the models

\footnotetext{
${ }^{12}$ We will encounter such models in the next chapter where we use it to model time constrained judgment and decision making
} 


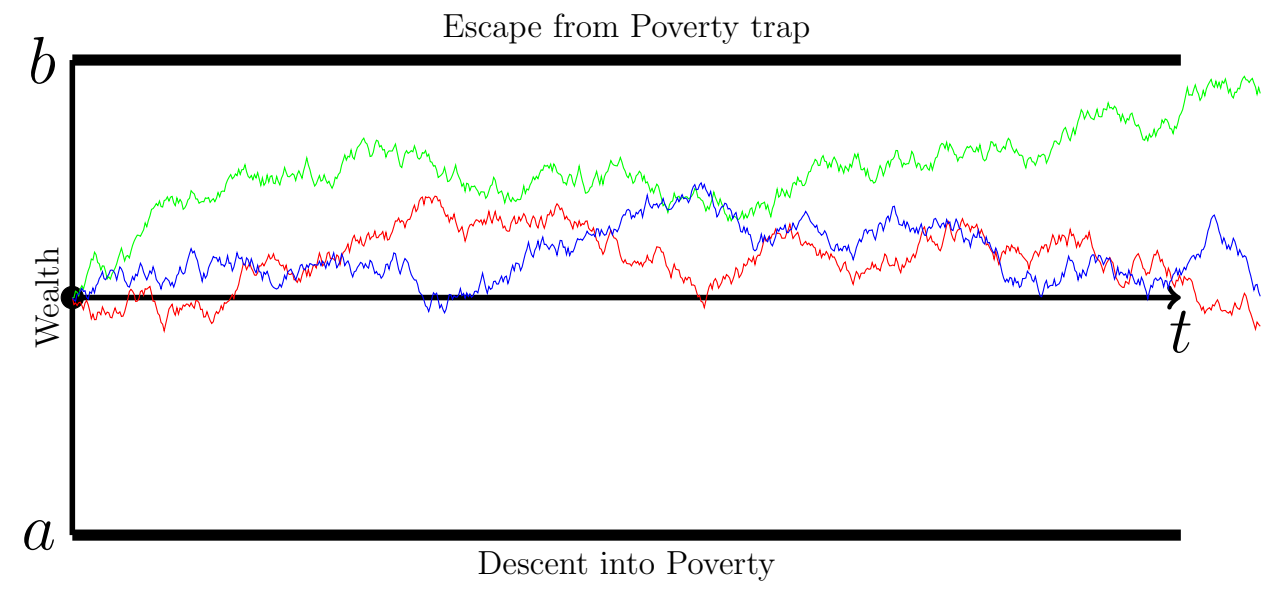

Figure 2.8: Model of poverty traps with a small drift component

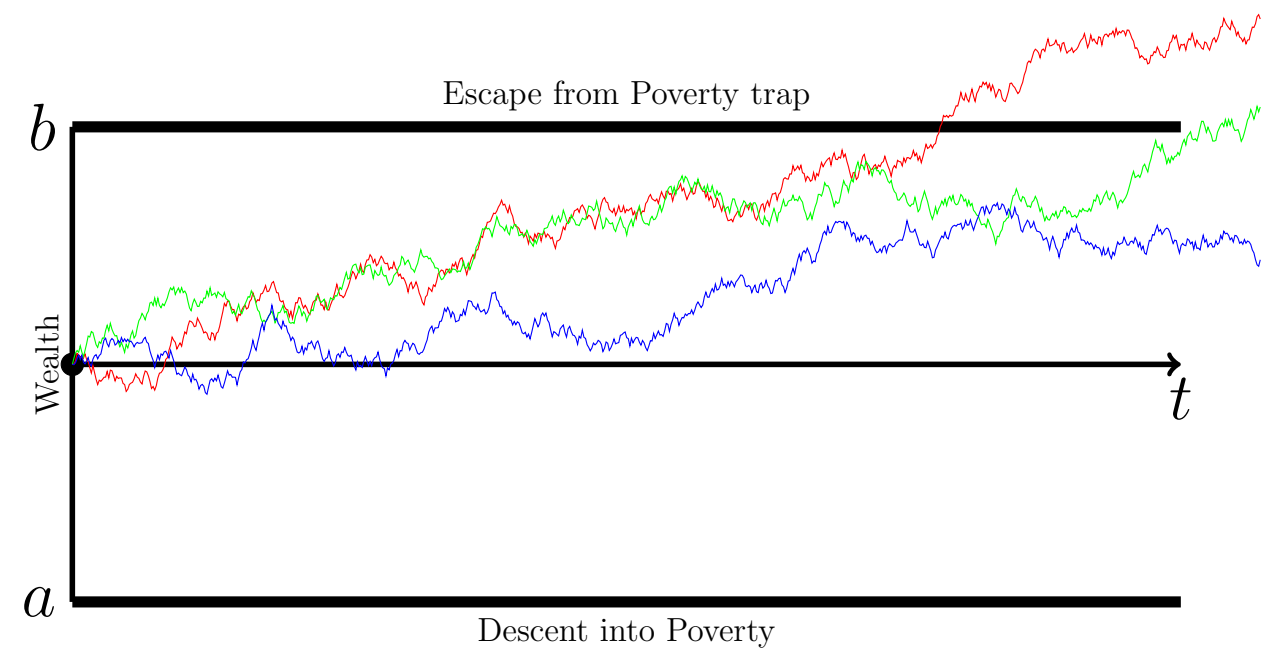

Figure 2.9: Model of poverty traps with a moderate drift component

in a qualitative manner referring back to the previous discussion when necessary. The models introduced above have three categories of parameters: diffusion coefficient $(D)$, drift $(v)$ and the length of the bounded interval $(L)$ on which the dynamics take place.

Suppose $x$ corresponds to wealth of an social actor. This social actor has a steady rate of growth of wealth $v$. This rate is an aggregate value that includes all sources of income and all sources of consumer commitments. For actors in middle 


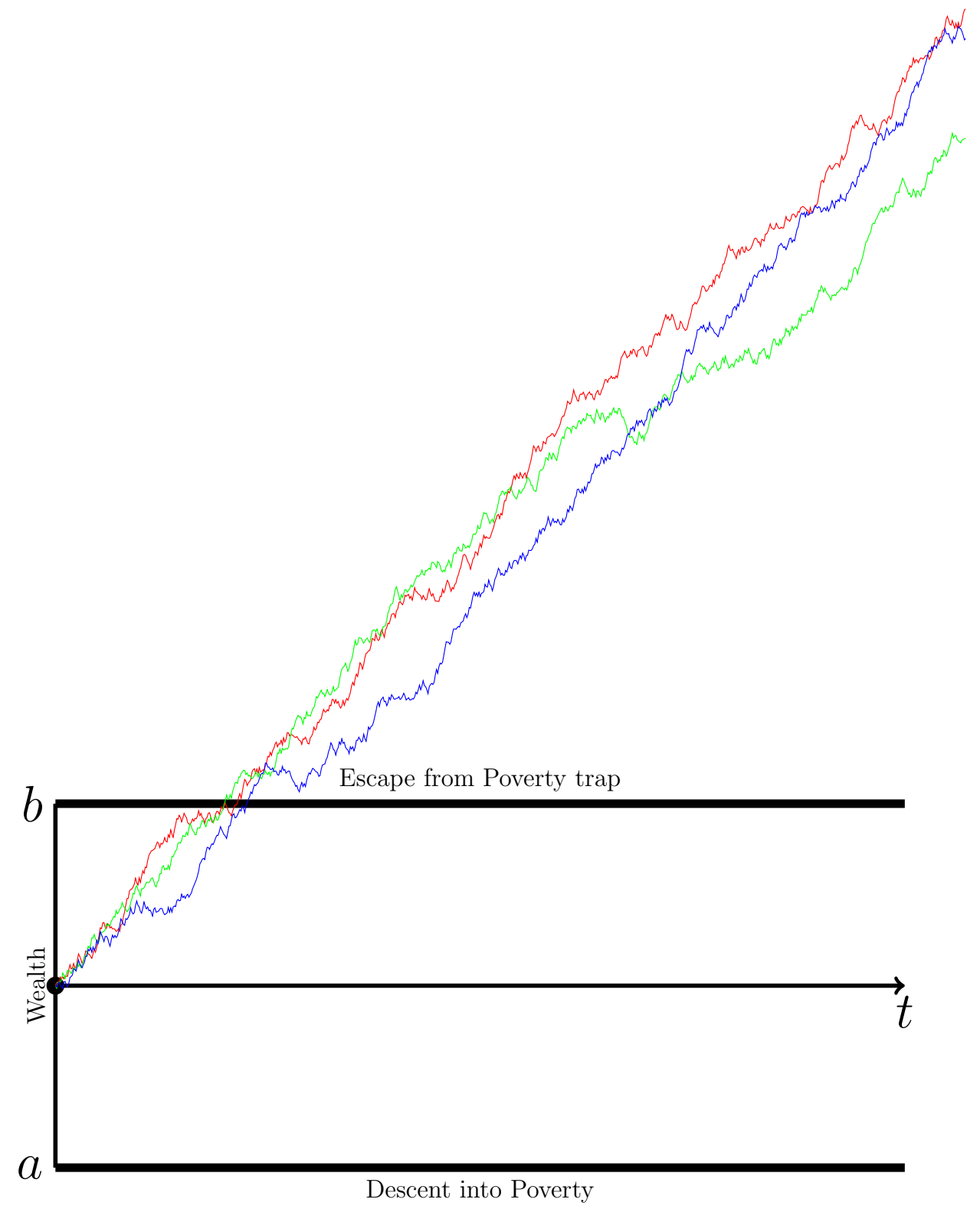

Figure 2.10: Model of poverty traps wirth large drift component

and higher socio-economic classes, the day-to-day or month-to-month shocks of random commitment shocks ${ }^{13}$. For simplicity we model this unanticipated commitment shocks as Brownian noise $D$. The boundary points at $x=0$ corresponds

\footnotetext{
${ }^{13}$ This corresponds to mundane events like sicknesses of actors themselves or their family members, transportation failures, etc.,
} 
to abject unrecoverable poverty and $x=L$ corresponds to the poverty trap threshold which the wealth threshold, if crossed, would allow the social actor to escape poverty permanently.

Now that we have imparted meaning to SDS, we revisit (2.13) and look at that equation and the associated system. MFPT presented there corresponds to a conditional exit time of reaching one boundary without reaching another. That is, the equation is an answer to the question: Given that an actor is observed with wealth $x$ at a start time, what is the expected time by which this individual is expected to escape abject poverty and cross the poverty threshold at $L$ ?

Suppose a social scientist were to perform a long term study of different neighborhoods within the same city using techniques pioneered by Sampson [109, 62, 110]. The neighborhood effects hypothesis of poverty traps suggest that different neighborhoods (say labeled by $i$ ) have different different unexpected consumer commitment shocks $D_{i}$. Given the same level of wealth growth rate, one could observe systematic differences between different neighborhoods. From the sample expected time of escape from trap, which could be leaving the neighborhood permanently, could be calculated. From this, one could estimate the $\hat{D}_{i}$. Urban sociologists who work with theories of place have developed various neighborhood level ecometric measures. If $\hat{D}_{i}$ is found to be correlated with other neighborhood ecometric measures, then one can make an argument for the inclusion of the MFPT based measure into the gamut of existing ecometric measures. This is a very testable claim assuming we have the necessary data at hand.

Figure (2.7) presents the basic model of poverty trap ${ }^{14}$. It models a case with no drift. Upon reaching $a$, the actor enters a state of permanent poverty.

\footnotetext{
${ }^{14}$ The notation presented in the figure is meant to be consistent with the rest of the chapters of the dissertation. In this chapter, the dynamics take place in $[0, L]$
} 
Upon reaching $b$, the actor enters a state of permanent escape from poverty. It is the average time to reach the boundary from a given point (MFPT). This is an important characteristic of escape from poverty dynamics.

In figures $(2.8,2.9,2.10)$, we present sample dynamics with drift turned on. Notice how the sample paths increasingly tend towards escape from poverty point.

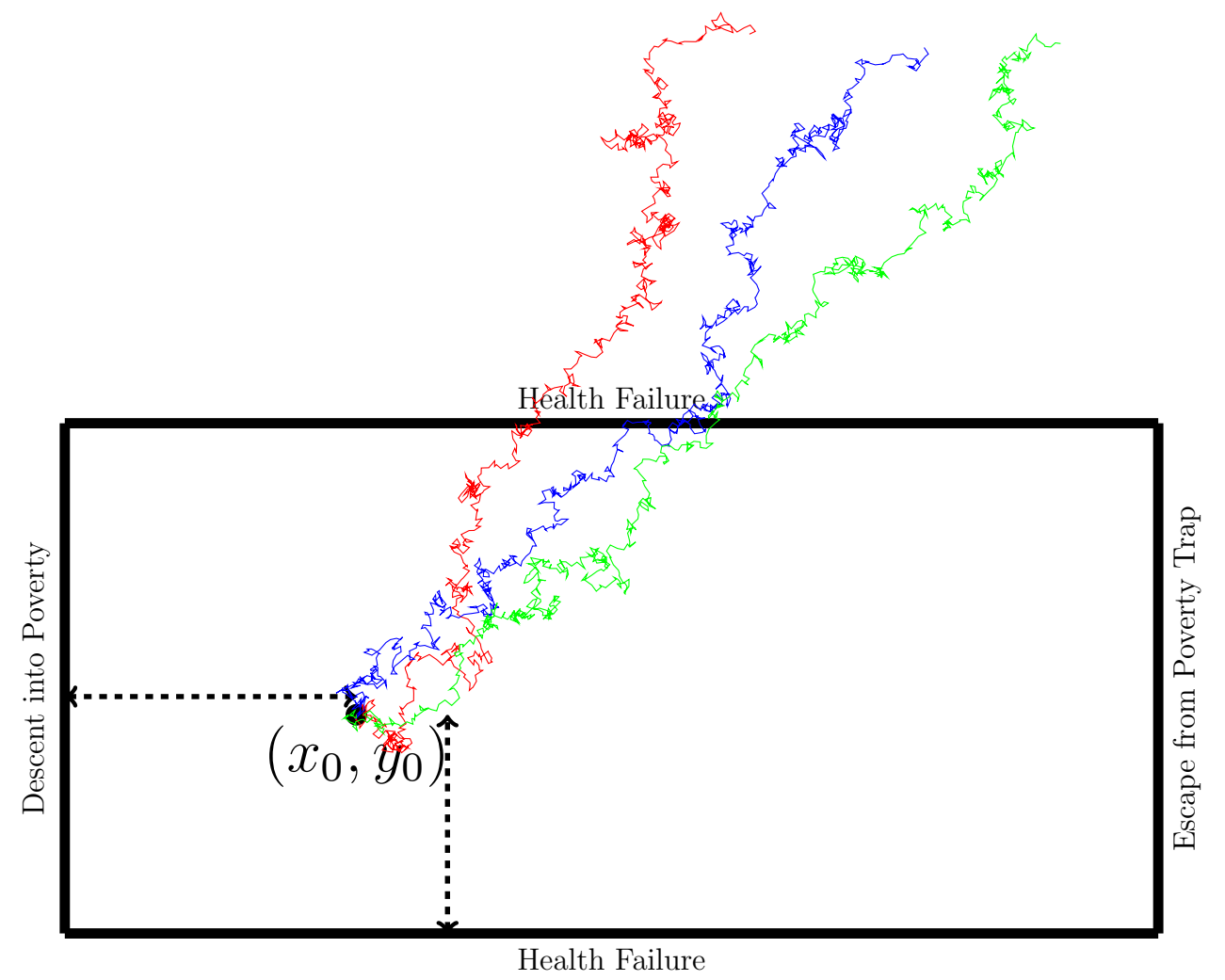

Figure 2.11: Poverty dynamics in the presence of health failures While the second dimension in this figure corresponds to health failures, the second dimension can correspond to other demographic variables too. Time is implicit.

In Figure ( 2.11), we present a simple extension of one dimensional dynamics to two dimensional settings. The process starts at a point $\left(x_{0}, y_{0}\right)$, an initial state of wealth and health. As the dynamics unfolds in time, depending on the two independent drift and diffusion constants in the two dimensions, the path may hit the failure zones before hitting the escape from poverty point. Using MFPT 
expressions presented earlier, relevant questions about these escapes from two dimensional traps may be answered.

This simple model can capture other realistic variants. The random consumer commitment shock $D$ could be a function of various social and demographic parameters. For example, family stability [17] is a known predictor of wealth inequality. Given what is known in works in urban sociology, it is reasonable to postulate that, after controlling for all other variables, whether or not an actor is divorced would predict the value of $D$. More specifically, $D_{\text {married }}<D_{\text {divorced }}$.

While other plausible models can be envisioned ${ }^{15}$, we continue our focus on models involving equation (2.13). While we could survey the rich literature on urban sociology for inspiration with the same basic empirical stance, we shift our attention towards what this analytical model in tandem with other computational social science approaches can offer.

Already, as will be discussed in the subsequent sections, more specific analysis of individual trajectories of social actors shows interesting complexity. Instead here, we speculate on a simulation project that could be undertaken as a next step. Previously, we emphasized the role of neighborhood effects in explaining or predicting escape out of poverty traps. One of the aggregate ecometric measures involve the actor's social network characteristics. In sociology, this is done in lieu of actually determining the social network structure because of the expense

\footnotetext{
${ }^{15}$ For example, consider in addition, the health status of an individual. For simplicity, let us collapse all aspects of health into one single variable. It is reasonable to assume that, like BMI, there is an optimal range of values. So, anytime an individual goes below or above the optimal range of values, the individual drops out of the escape from poverty process because of poor health. In the literature, health transitions are modeled using event-history analysis [75]. Since these are related to CTMC, this can be modeled as yet another diffusion with or without a drift process, just like our original transition from CTMC, to a diffusion processes with absorbing boundaries to model the dropping out of the mobility evolution process. We model dynamics as a two-dimensional drift-diffusion in section (2.3.2), then we could use the analyze above to calculate the conditions under which individuals are able to escape from poverty before a health failure.
} 
nature of network sampling exercises. These are natural questions addressed by both computational social science and analytical sociology [64, 76].

Consider the original model defined in (2.1). Now suppose there are $N$ social actors that all satisfy their own wealth evolution equation

$$
d x_{i}(t)=v_{i} d t+\sqrt{2 D_{i}} d w_{i}
$$

Further, suppose the $N$ actors are part of a social network corresponding to the graph $G$ with adjacency matrix $A_{i j}$. Potentially both $v_{i}$ and $D_{i}$ could be functions of network characteristics. As a preliminary set of models, a simulation on simple reference networks like the one discussed in [94] could be used. Typically, in networked dynamical systems, both the node level dynamics and the global dynamics are mediated by network characteristics like centrality measures, modularity measures, graph Laplacian, etc. The interplay of drift-diffusion dynamics of network structure might produce interesting results.

One could do better also by constructing networks using models of social processes. As we might recall, the ecometric measures are aggregates for neighborhood explanatory factors. Some of these factors correlate with known social forces of empirical and theoretical import like homophily ${ }^{16}$ [89], consolidation $^{17}$ [88], and social cohesion $^{18}$ [50]. Social network models can be generated based on specific values of these social forces and the results from the simulation can be compared

\footnotetext{
${ }^{16}$ Homophily is the tendency of social actors with similar social and psychological attributes to form stable relationships. The are important in understanding social structure formation and maintenance.

${ }^{17}$ In modern societies, social, economic and cultural roles of actors tend to overlap. This overlap creates dense clustering of social relations. One consequence of consolidation is the tendency of highly clustered societies to repel or discourage relationships from actors not belong to such cross-cutting social circles.

${ }^{18}$ Cohesive forces are forces that are catalyzed by synchronized behavior. Actors whose behaviors are synchronized -performing a social ritual together, for example- tend to form social ties, even if they do not share any homophilous traits.
} 
with purely ecometric models. A similar experiment could be performed in the presence of social influence ${ }^{19}$.

\subsection{Conclusion}

Stochastic dynamics thus offers a novel model class useful for discussing social processes; this novelty is despite their well-known use of such models in physical and engineering sciences. Interestingly, these models have not been popular even in the economics literature as questions about income and social mobility are dealt with at the coarse grained macroscopic level. Since we discussed sociology literature in the earlier section, we conclude the chapter by shifting our focus towards economic models.

In macro and micro economics, the concept of representative agents is a pervasive one. These are agents constructed to be idealized versions of economic actors. Typically, results in macro and micro economics are derived by maximizing the utility functions of such agents. Despite their limitations, such models continue to be the basis for economic theory in spite of their overly simplifying assumption of homogeneity of representative agents. Not only that, these representative agent models are all deterministic in nature. Only recently, heterogeneous agent models [2] based on stochastic components have been proposed as alternatives. Solutions

\footnotetext{
${ }^{19}$ Recently, there has been a lot of focus on social simple and complex contagion [30]. Suppose $D$, the exogenous commitment can be decomposed into two terms $D=D_{i}+D_{\text {norms }}$. Furthermore, suppose the norms can spread through a given social contagion model where ideas spread as it it is a disease carrying contagion. As is observed in the literature, the structure of the social network shapes the spread of norms and conventions. And this indirectly influences escape from poverty traps of individual actors. Since such interventions have been seen to be effective in spreading various health related and other economic habit forming behavior [72], these experiments can be useful proxies for whether one could use social network based interventions to enable actors to get out of poverty traps more efficiently than with other conventional policy interventions. For example, the work by Centola [30] suggests that healthy habits can be transmitted only in certain kinds of social networks. Similar experiments may be possible for policy interventions for promoting escape from poverty.
} 
to these models are based on Fokker-Planck partial differential equations. So, despite their pervasive use in physical sciences, the one-dimensional models introduced in this chapter are state of the art in economics and sociology. More importantly, even in these heterogeneous agent models, finite time transients and constraints are not discussed; instead they are constructed to model economic processes at a macroscopic scale. To the best of our knowledge, the models proposed in this chapter have not been used to study poverty traps, making it an important contribution of this dissertation.

The models proposed here have other secondary roles to play, especially as models of social processes. They provide insights that sometimes confirm and at other times refute commonsense explanations of social phenomena. For empirically oriented researchers, these computational models can help researchers to avoid attributing population-level noise to causal narratives. Specifically, the parameters of these diffusion models, especially the first-passage time distribution may become useful measures of dynamics of mobility and escape from poverty. Even with simplifying assumptions, the models are able to capture key many aspects of mobility dynamics like diversity in individual histories. FPT measures are one of several mathematical measures used to describe diffusions and other Markov processes. Like FPT, we expect those measures to also be useful measures of mobility dynamics.

Also of theoretical interest is use of dynamical systems as a way to expand on the scope of traditional agent-based modeling approach common within computational social science. Interestingly, dynamic process models are more used in analytical sociology as a way to encode social mechanisms. The current model affords a two-way interaction between computational social science and analytical sociology $[64,76]$ that is argued to be necessary for sociology and social science 
in general, with AS structuring models in CSS, and with CSS models providing mathematical and computational realizations of social mechanisms concocted with AS.

While the models of poverty traps and escape from poverty dynamics make sense as a continuation of literature on models of individual social processes, it is worth pointing out that the dissertation arrived upon these models through the idea of lifting biological reliability and projecting onto social processes. The fact that all of the biological notions of reliable behavior along with quantitative models of those concepts were transferable to social concepts and models is a vindication of systems approach espoused in this dissertation. In the next chapter, we do the same, but to understand models of dynamic judgment and decisions. 


\section{Drift-Diffusion Models of Bias, Uncertainty and Conflicts in Judgment and Decision Making}

\subsection{Introduction}

This chapter

1. Introduces two new models to the drift-diffusion model literature

2. Links drift-diffusion models with models of ecological rationality

In this chapter, we introduce two variants of drift-diffusion models(DDMs) and suggest potential judgment and decision tasks that may utilize the mechanisms underlying these models. Due to presence of multiple scales and levels, these models exhibit interesting first-passage dynamics. Inspired by sub-neuronal biology and by theoretical speculations, the associated stochastic accumulator dynamics displayed here could motivate us to look for such features in existing experimental data. We suggest the use of these new variants in modeling biased judgment and decision tasks.

Like the contributions discussed in Chapter 2, the systems approach of lifting and then applying the concept to unrelated domains leads to two distinct kinds of contributions: suggesting models to DDM literature; connecting unrelated subfields. The new models could -with some effort- be motivated directly, without having to invoke the idea of systems isomorphisms. However, the connections between DDM models of noisy accumulation and ecological rationality -missed by 
the literature- is made possible only because we framed noisy accumulation-based dynamic judgment and decision processes as reliable in a lifted sense, allowing us to connect in the idea of Simon and Giegerenzer's concept of satisficing [52].

A DDM attempts to capture the idea of imperfection, temporality and dynamics associated with everyday judgments and decisions. The imperfect nature is captured by both a local stochasticity (Brownian noise) and a global stochasticity -captured by the qualitatively different exit paths of a stochastic process. The temporality and dynamics are captured by the generative mechanism underlying a stochastic process. In one-dimensional setting, the DDM corresponds to a dynamic system with state space lying on an interval, a steady drift and Brownian noise. Taken together, it captures the idea of imperfect accumulation of evidence as the end point of such a dynamic system could be at 0 or at 1 . Such a model was first proposed by Ratcliff [102] to model the imperfect nature of memory retrieval.

Since Ratcliff's original construction [102], drift-diffusion models ${ }^{1}$ have been extended and generalized along various dimensions [126]. Response time distributions associated with DDM have been used to characterize differences in cognitive performance metrics of control and stratified test populations. They have been used to study group and inter and intra individual differences in aging, anxiety disorders, ADHD, etc., and in clinical populations. Furthermore, recent works use DDM and its generalizations to lay a dynamical process based foundation for random utility theory and stochastic choice and order theories ${ }^{2}$. More generally, building on DDM based random utility models [28], these models provide founda-

\footnotetext{
${ }^{1}$ Although we abbreviate drift-diffusion models to DDM, in the literature, diffusion-decision models have also been abbreviated to DDM. Even though these two senses are mostly synonymous, we prefer the former sense as it better reflects the nature of the model.

${ }^{2}$ Traditionally, both order theory and utility theory do not encode probabilistic knowledge. Both stochastic order theories and random utility theories incorporate probabilistic knowledge by making binary relations and preference ordering based on a probability distribution.
} 
tions for rational information processing models of neuroeconomics. Motivated by these generalizations and expansions in scope, we propose two variants of DDM and suggest their value for modeling existing and novel dynamical decision and judgment tasks.

Luce's work $[85,84]$ on response time data and its emphasis on using response time models to infer and reconstruct mental organization is an important precursor to DDM, and more generally, to work in sequential sampling models [47] and model-based cognitive neuroscience [48] [46]. Identifying design principles underlying judgment and decision making, and cognition more generally continues to be of interest, be it in the pragmatic approach of Simon [119], or in the programmatic approach of Marr [87]. While we discuss Simon's approach later, we briefly discuss Marr's Levels.

Marr's original suggestion was to organize cognitive neuroscientific explanations in terms of levels: a computational level, a representational level, and an implementational level. Further, he insisted that focus must be directed towards explanations and integration of all three levels Computational level analyzes the system in terms of the problems they solve; algorithmic or implementational level studies the processes by which the problem is solved; and the implementation level addresses questions regarding actual physical instantiation of representations and algorithms.

A predominant school of thought interprets the computational level constitute search for universal principles of representationalistic cognitive psychology, but others point out limitations of this interpretation as it neglects time and dynamic self-organizational and coordinative aspects of cognition, especially with a focus on behavior. Such alternative approaches [112] tend to view the highest of Marr's Levels as questions surrounding an architectural blueprint of how 'satis- 
factory' behavior is enabled by the underlying cognitive processes. DDMs, with their emphasis on trade-offs between response times and accuracy fall under this alternative interpretation.

This dissertation was conceived of in an attempt to understand reliable behavior of biological agents. Although not emphasized in the literature, we argue later that DDMs are models of theories of ecological rationality [130, 22] where concepts like response times explain performance relative to ecological structural constraints. It attempts to build upon Simon's concept of satisficing in which judgments and decisions made by agents are always understood relative to the characteristics of the agent's environment, in contrast to absolute optimality based principles in classical theories of decision making. From this perspective, DDM are dynamical constraint satisfaction models. Setting aside our conceptual motivations in rationality and satisficing, the models introduced in the next section can be motivated more simply as an exercise in model-based cognitive neuroscience. This is in contrast with more mathematical approaches that use combinatorial theory $[39,106,45,93]$ and that avoid dynamical models.

The first generation of DDMs were developed as empirical models to explain response time data. Since then, advances in neuroscience, and the need to integrate findings across different levels of the neural information processing system, have resulted in different motivations for the next generation of DDMs. Modelbased cognitive neuroscience offers a commonsense approach towards integration of empirical and theoretical knowledge spanning multiple levels. For example, statistical models used to explain cognitive neuroscientific data can incorporate ideas from mathematical psychology, allowing for more expressive empirical models. Similarly, empirically identified mechanisms in neuroscience can serve as inspirations for new models in mathematical psychology, allowing for synthesis 
with existing formally constructed models. This attitude is reflected in the literature where sequential sampling models and DDM are used to model static judgment and decisions even though traditionally judgments, decisions, and actions are perceived as static tasks not requiring dynamics. This reflects the increasing confidence in the use of dynamical process based models as the right approach to cognitive models because the the underlying neural mechanisms are themselves dynamic. Similar justification has allowed the use of DDM to interpret data from EEG and fMRI experiments. Given such motivations, DDM and sequential sampling models have been generalized along different dimensions and found uses beyond the original scope. Apart from more direct extensions like making the decision boundaries dynamic, drift and diffusion coefficients non-stationary, other conceptually significant generalizations have been made, both in cognitive psychology and cognitive neuroscience. For example, DDM are in use as models of multi-attribute, multi-alternative [78] and value-based decisions [28] not only in psychological tasks but also to identify neural mechanisms and to localize neural correlates statistically in neuroscientific experiments [99]. DDMs are also in use to study one-choice tasks [104] and to study models in neuroeconomics [73, 134, 133] and moral judgment tasks [37]. On the mathematical side, far reaching generalizations have been made, extending the underlying dynamical state space to higher dimensions [124, 125] and even to infinite dimensions [103].

Aligned with the goals of model-based cognitive neuroscience, and with the major themes of this dissertation, we observe that the generative mechanisms underlying DDMs are present in neural processes $[55,66]$ across at scales of space and time, from whole brain electrical activity to sub-cellular organelle level biophysics. A deeper scrutiny of these processes reveal generative mechanisms that can be modeled by structured generalizations of diffusion processes. In particular, 
more general diffusion processes like piecewise-deterministic Markov processes and multi-timescale diffusion processes are possible. Inspired by them, we lift these models out of the sub-neuronal realm and cast them as generalizations of DDM, fit for use as sequential sampling models.

The two models introduced in this chapter illustrate this idea of lifting that we introduced in Chapter 1. In other DDM extensions, the models were constructed as interesting extensions of existing DDM models. In contrast to models motivated by response time experiments, the lifted models have the distinguishing feature of being models of nano and micro physiology. One model [86] was recently constructed in order to understand the movement of bacteria; the other model [66] is a model of biomolecular transition often used in modeling sub-cellular physiological processes. The use of such biophysical models to construct DDM is a contribution of this dissertation.

In the next section, we introduce these two models and discuss their potential uses for modeling psychological processes involving sequential sampling models and We conclude in the following section by discussing other extensions inspired by this approach of borrowing models from nano and micro physiology.

\subsubsection{Connections to models of Chapter 2}

The basic DDM introduced by Ratcliff is identical to the models of intragenerational mobility presented in Chapter 2. Both are based on FPT analysis on an interval. The key differences between the mathematical sociology application from the previous chapter and this application lie in their pragmatic value. While the both models are good reference models, the models of the previous chapter are deductively used; this is in contrast to the inductive use here as good explanatory models of response time distributions. As is practiced in empirically oriented 
cognitive psychology, where experimental design issues constrain how model or hypothesis space are enlarged, researchers rightly focus on addition of one or two explanatory factors at the time. While optimal within the field, it restricts itself to a narrow class models. However, a theoretical orientation inspired by nano and micro physiological process models suggests new model classes not discussed in the literature.

MPFT equations from Chapter 1 is identified with response time distribution's statistical mean under various experimental protocols. Specifically, the variants of DDM as cognitive psychological models are isomorphic to models of poverty traps and the dynamics of escape from poverty traps. This strategy of repurposing models from one discipline for use in others by careful identification of isomorphisms between systems is extremely potent. We will continue to do this in the next chapter when we sketch work in progress where the same kind of FPT and MFPT analysis is used to model biological behavioral primitives like fixed action patterns and startles.

One of the goals of this chapter is to use the models introduced here to discuss how probabilities of choices made in two alternative forced choice (2AFC) experiments and other related protocols would change if different alternative drift and diffusion terms are used. In 2AFC experiments, experimental subjects are presented with two choices, only one of them is the correct choice. Furthermore, the subjects are pressed to make their choice as soon as possible. This creates a scenario where response-accuracy trade-offs can be studied. Data in such experiments consist of the choices made and their associated response times.

For this purpose, we have focused on $E(x)$, the expected probability, starting from $x$ to reach one of the two decision boundaries. While we can perform a similar analysis with MFPT $t(x)$, we focus on $E(x)$. In addition, the second 
of the two models introduced here requires asymptotic techniques like WentzellKramers-Brillouin approximations and is mathematically different from the models presented in Chapter 2. In such models, even in simplest of cases, a proper analysis of MFPT in those scenarios requires the use of techniques from rare-event simulations. Since we were more interested in the establishment of systems isomorphisms, we did not focus on this aspect of the analysis and it is the most natural next step in our future work.

The models presented here are different from traditional DDM. For example, DDM are fit assuming that the models contain drift and diffusion terms that are constant or slowly varying piecewise linear functions of time. This leaves the MFPT approximately the same as the ones for the finite interval case. The models in such cases are identical to the ones presented in Chapter 2. However, in the models presented here, drifts are either stochastic or nonlinear. This has implications for FPT characteristics. We note this in the subsequent sections of the chapter by pointing out differences with conventional DDM with simple drift and diffusion terms.

\subsection{Models}

In what follows, we present two incremental extensions: one adds stochasticity to the drift via a continuous time Markov chain (CTMC); the other adds nonlinear deterministic structure that adds an additional timescale into our analysis. These extensions are used as models of biased judgment and decision making by linking them to experiments in cognitive neuroscience.

Sequential sampling models are a plausible and attractive way to think about how agents make judgments and decisions in cognitive tasks with response times 
(RT) - the time taken for judgments and decisions to be made being of fundamental experimental importance. The sampling for evidence, if it happens in continuous-time, collapse to DDMs. Since evidence accumulation can be thought of as a continual perceptive process, an interesting comparison made with with signal detection theory (SDT), in which perception happens instantaneously. DDMs are tied to a dynamic sampling and evidence accumulation over time unlike static exposure assumptions in SDT and general recognition theory [4] (GRT). All the same, the generalization of SDT and GRT to multidimensional manifold setting parallels extensions proposed here and in discussions of future work. The models presented here and elsewhere can be thought of as dynamical analogues of Fechnerian psychophysics.

Fechner's law [4] is a phenomenological feature observed in psychophysics experiments. In such experiments, the perceived difference between two stimuli of different magnitude is logarithmic rather than linear. This nonlinear relationship between physical magnitude of the stimuli and the perceived intensity can be generalized for multidimensional stimuli and pereception spaces. The generalized Fechner's law is then posed in differential geometric terms. In such a generalized experiment, the result is attributed to differences in metric structure of underlying psychological spaces. GRT is a generalization of Fechner's laws of psychophysics where the psychological spaces are postulated to be non-trivial Riemannian manifolds. While GRT models encode stimuli as static points, DDM are dynamic process versions of signal detection models; instead of a single point on a non-trivial Riemannian manifold, it is a stochastic path on a non-trivial domain.

Irrespective of its historical connections [102, 85, 84] with sequential statistical decision theory, DDM's current attractiveness is its association with modeling of cognitive processes, motivated by neural mechanisms. This connection to 
underlying neural processes directly informed the construction of the models to be discussed in this chapter. Although discussions of DDM can be made with greater generality ${ }^{3}$, we focus on a one dimensional DDM here. Since the focus of this chapter is theoretical in nature, we do not discuss model fit here ${ }^{4}$.

The boundary of the domain, $\mathcal{D}$ represents the decision criteria, the threshold for evidence for a decision or judgment task. The drift $\mu(x(t), t)$ parameter represents evidence accumulation. $\sigma(x, t)$ corresponds to the noisy nature of the evidence accumulation process. Since, the state variable $x(t)$ represents the psychological measure of evidence, representations of noise and evidence accumulation need to be clearly delineated in generic DDM. Sometimes, non-decision components and associated latent response times are incorporated into the model as additional parameters to match the specifics of the psychological process. Nonstationarity can be encoded in the functions defining the SDE and in the boundary as well. The resulting evolution equations become harder to solve but still

\footnotetext{
${ }^{3}$ The mathematical model of accrual of evidence for a judgment or decision in DDM are modeled as stochastic differential equations (SDE) as follows [123], discrete-time and discretestate versions of DDM, although important, are not not relevant, and hence not discussed here.

The original diffusion model $[102,126]$ assumes that dynamic evidence accumulation leads to one of two correct decisions or judgments. The evidence accumulation is considered to be noisy, but with a steady drift in the direction of the correct response, but errors may occur, even within multiple trials with the same subject, controlling for all other variables. The evidence accumulation state space is an one dimensional interval with boundaries corresponding to the two decisions that a subject could make. The times to decision under changing experimental conditions is the basis for subsequent inferential arguments about the mental organization underlying such decision making tasks.

$$
d x(t)=\mu(x(t), t) d t+\sigma(x(t), t) d B(t)
$$

where the functions $\mu($.$) and \sigma($.$) are the drift and diffusion coefficients for the state space X$ variable's evolution over time with the decision or judgment deemed to have taken place once the state exits the domain $\mathcal{D}$ via the boundary $\partial \mathcal{D}$. $\tau_{D}$ is the response time, the time taken for the process to cross the decision threshold. Analytical calculations of response time is calculated via an associated Fokker-Planck equation. We keep the SDE, associated domain and boundary general as DDM are no longer confined to the restricted models historically constructed to model 2AFC experimental data. Typically, the goal of DDM is to explain data from experiments.

${ }^{4}$ We refer the reader to packages like HDDM, fast-dm and DMAT for more details.
} 
tractable by numerical means.

The analytical properties are typically not emphasized in the literature because of its peripheral relevance to model estimation and fit; something that is usually done using the statistically oriented martingale approach [126]. That is, precise analytical structure of solutions of DDM models are not emphasized; only an expectation value based analysis is conducted. Since our proposals are theoretically inspired, analytical approach is more suitable as it is used to derive FPT and MFPT of associated SDE. This also makes the isomorphisms more manifest in terms of MFPT.

In one dimensional settings, equations are as follows

$$
d x(t)=v d t+\sqrt{2 D} d w
$$

One can show that the solution of the stochastic dynamical system satisfies the diffusion equation ${ }^{5}$ with drift $v$ and diffusion constant $D$.

$$
\frac{\partial c(x, t)}{\partial t}+v \frac{\partial c(x, t)}{\partial x}=D \frac{\partial^{2} c(x, t)}{\partial x^{2}}
$$

where $c(x, t)$ is the one probability density function. When drift $v$ vanishes

$$
\frac{\partial c(x, t)}{\partial t}=D \frac{\partial^{2} c(x, t)}{\partial x^{2}}
$$

The MPFT $t(x)$ satisfies another $\operatorname{PDE}^{6}$

\footnotetext{
${ }^{5}$ The equation follows from the fact that diffusion processes are all Markov processes and hence must satisfy Kolmogorov's forward-backward equation

${ }^{6} \mathrm{We}$ encountered this in the previous chapters and will encounter the same equation in the models to be presented in the subsequent sections of this chapter but with different state spaces and boundary conditions.
} 


$$
D \nabla^{2} t(x)+v(x) \nabla t(x)=-1
$$

The two proposed variants are extensions of these one-dimensional models. In the first variant, we add a stochastic switching component, $v$ can change signs at the rate induced by a simple $\mathrm{CTMC}^{7}$ with a Poisson rate $\gamma$. In the second variant, $v=-U^{\prime}(x)$ where $U(x)$ is a multimodal function, so drift now is not a trivial constant. Theoretical response times can be calculated for both these cases, sometimes using asymptotic approximations.

\subsubsection{Classical DDM}

In this section, we present the original Drift-Diffusion Model [102, 123], and present some known properties of response times of such processes.

Conceptually, DDM (figure 3.1) is identical to the model from chapter 2 where we introduced the drift-diffusion model as a model of escape from poverty. As is customary in the literature, the process starts at a time $t_{0}$, at the initial evidence point $x_{0}$. Response time and accuracy is dependent on the starting point $x_{0}$ as well as the diffusion constant $D$ and the rate of information accumulation $v$.

For example, subjects in one such experiment [47] are asked to judge the general direction(right or lef) of a collection of moving dots. The drift rate represents the magnitude of evidence accumulated per unit time, and is considered to be an index of task difficulty and subject capability. The separation between the boundaries represent the degree to which subjects can be cautious: greater the separation, greater the accuracy in the given task, fewer the errors. This way a

\footnotetext{
${ }^{7}$ Like the discrete-time version, transitions to the next state depend only on the transition matrix. In addition, because we are dealing with continuous time, the transition from one state $i$ to another state $j$ follows a Poisson process $\lambda_{i j}$
} 
trade-off between accuracy and speed is established. The starting point of the diffusion can be thought of as the bias. In some cases, there is a non-decision time corresponding to predictable lags in the start of any cognitive process. All these parameters together completely specify a given DDM.

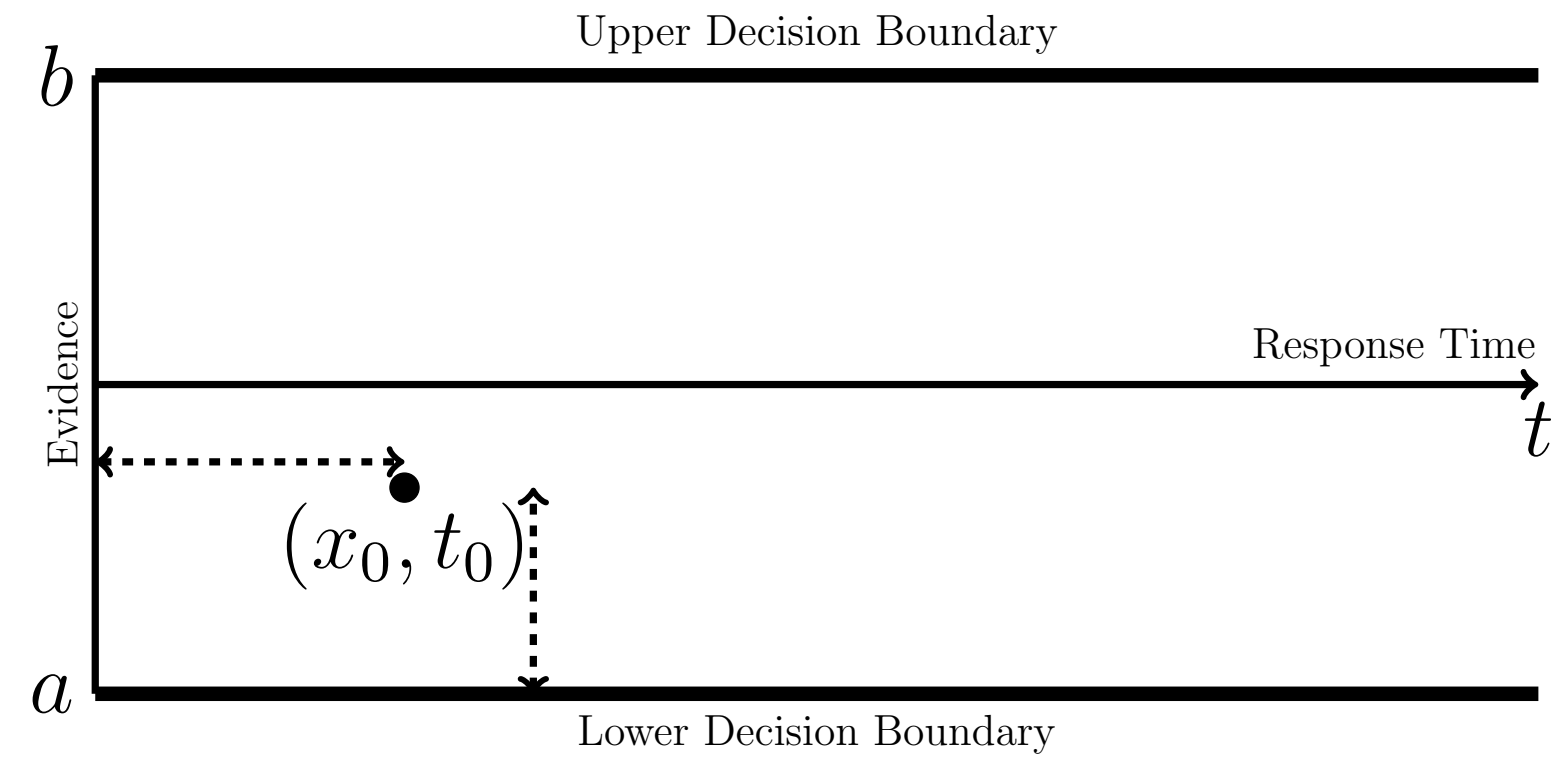

Figure 3.1: Drift-Diffusion Model

Solutions of Fokker-Planck equations associated with first-passage times of certain simple processes can be calculated as an infinite series. Fast numerical routines are available $[43]^{8}$ for certain kinds of diffusion models. While the processes from Chapter 2 have FPT densities similar to ones plotted here, since the focus was on economic applications, expectation values were more important as measures of wealth growth. In DDM, the response times to reach various decisions constitute the data and it is more natural to look for FPT density. However, as the DDM gets more complex, MFPT bases analysis is more prevalent. In appendix (), we present a brief introduction to parametric inference of DDM given data.

${ }^{8} \mathrm{We}$ used publicly available julia code to generate all FPT plots
(https://github.com/DrugowitschLab/DiffModels.jl)




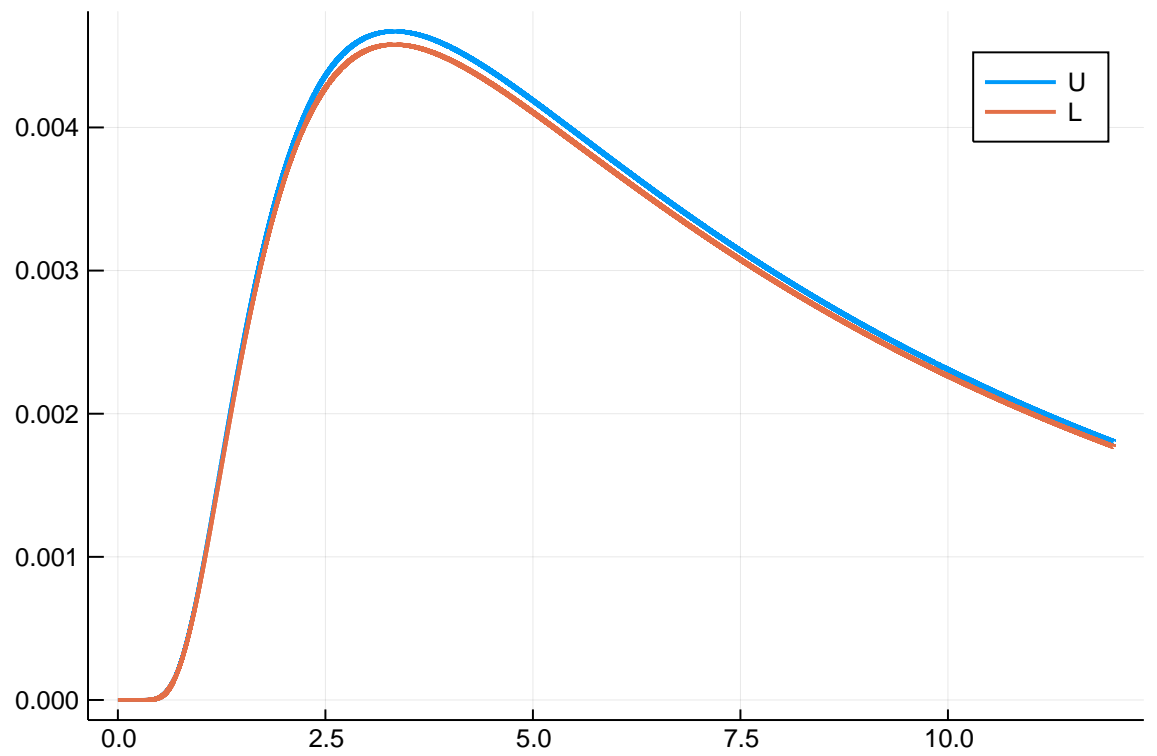

Figure 3.2: DDM with (almost) pure diffusion starting at the center $\mathrm{U}$ and $\mathrm{L}$ correspond to upper and lower decision boundaries. For convention, $\mathrm{U}$ is assumed to be the right choice in a $2 \mathrm{AFC}$ experiment. $x$ axis denotes time and $y$ axis denotes first-passage time probability density.

In Figure (3.2) is based on a simulated FPT density of a simulated diffusion process. The process was started at the mid point with no drift term. Curves U and $\mathrm{L}$ in the figure correspond to FPT density for reaching the upper and lower boundary. We artificially biased it infinitesimally to show the symmetric nature of the two distributions. Without the bias, the two densities will be identical. Next we move the starting point closer to the lower boundary.

In Figure (3.3), we plot a DDM with an asymmetric starting point. Since the starting point is closer to the lower boundary L, the paths lead faster out of the lower boundary. The sharp peak in response time very at short time confirms this expectation. Next we discuss variations in FPT density as we change drift parameters.

In Figure (3.4), we plot FPT densities for DDM with varying drift (informa- 


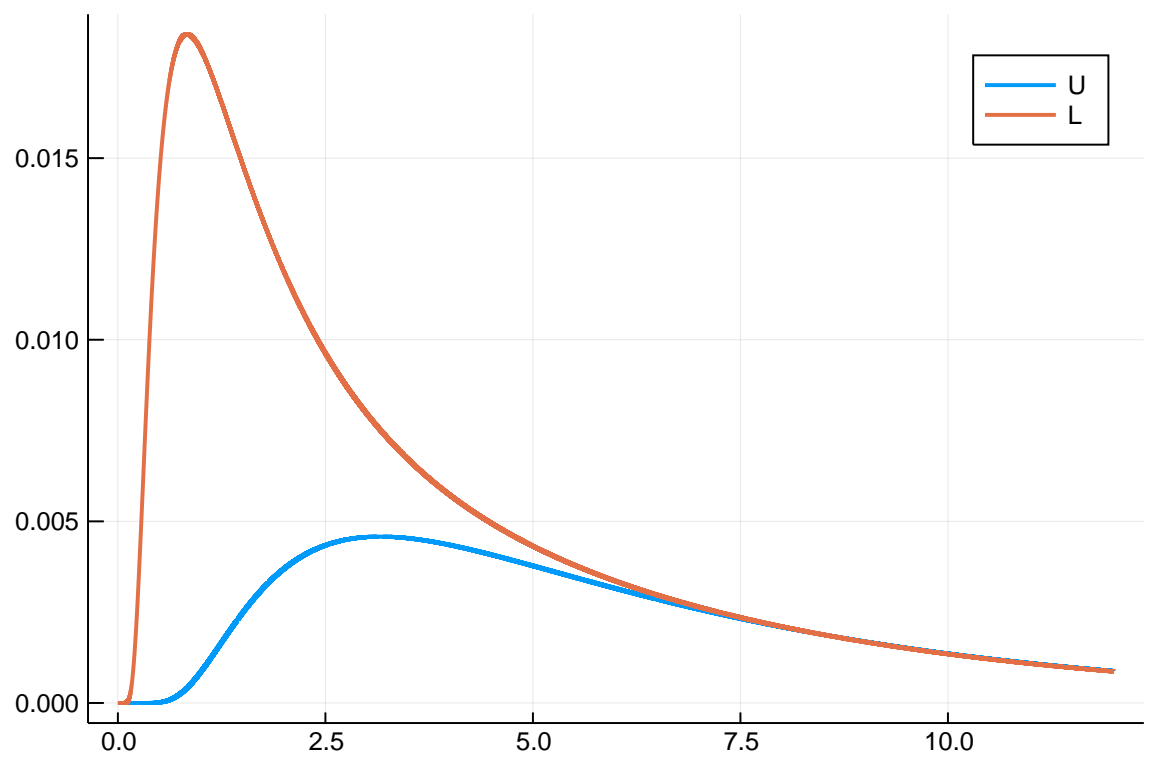

Figure 3.3: DDM with (almost) pure diffusion starting at the center $\mathrm{U}$ and $\mathrm{L}$ correspond to upper and lower decision boundaries. The initial starting point is asymmetric, starting closer to the lower boundary. $x$ axis denotes time and $y$ axis denotes first-passage time probabilty density.

tion accumulation rate) parameters. All curves correspond to FPT densities for reaching the upper decision boundary. When drift is positive (blue), curves tend to develop sharp peak and have earlier response times. In contrast, negative drift produces shallower peaks but later response times. Next we discuss the nature of FPT peaks by increasing drift to very large values

In Figure (3.6), we plot FPT densities to reach the upper boundary for various values of $v$. As is expected, as the magnitude increases for negligible to higher and higher order of magnitudes, the FPT density peaks shift towards the left and is increasingly sharp. For large values of $v$, drift dominates over $D$. 


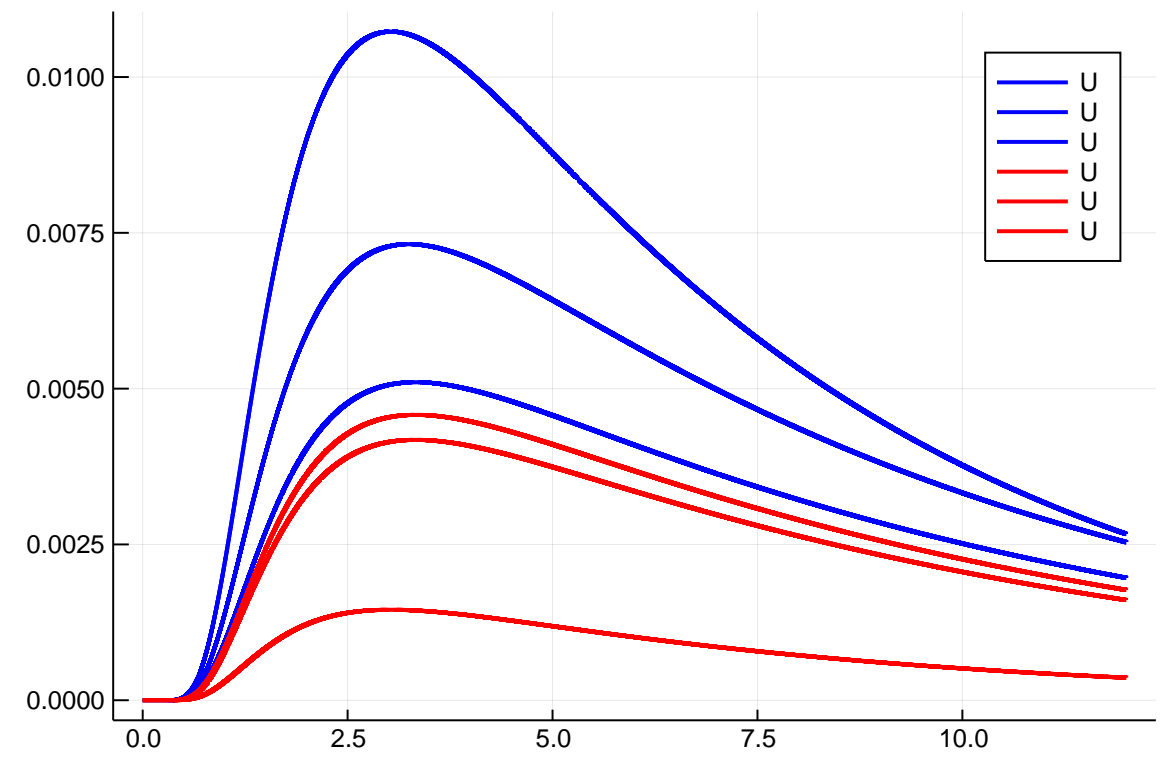

Figure 3.4: DDM with (almost) positive and negative drift

$\mathrm{U}$ and L correspond to upper and lower decision boundaries. FPT densities in blue correspond to positive drift towards U. FPT in red correspond to negative drift against

U. $x$ axis denotes time and $y$ axis denotes first-passage time probabilty density.

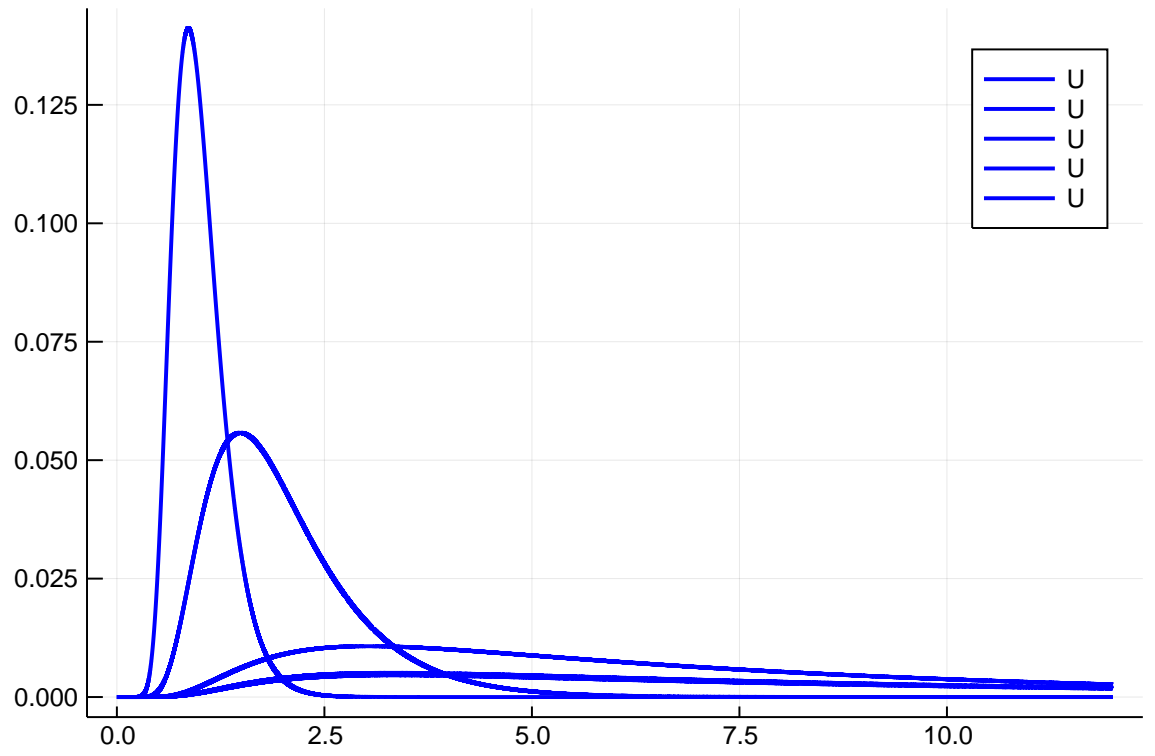

Figure 3.5: DDM with positive drift of increasing magnitude

$\mathrm{U}$ and L correspond to upper and lower decision boundaries. FPT densities in blue correspond to positive drift with increasing magnitude. $x$ axis denotes time and $y$ axis denotes first-passage time probability density. 


\subsubsection{Models with stochastic drift}

One dimensional drift-diffusion and FPT characteristics have been extensively studied in the physics literature [105]. Recently [86], a simple one dimensional model of movement of run and tumble (RTP) bacteria and active particle was constructed and studied for their FPT properties. RTP particles tend to move in approximately deterministic fashion, occasionally changing direction. [86] made simplifying assumptions, modeling the run portion as a drift-diffusion and the occasional tumbling as a CTMC. Such stochastic hybrid models are frequently used as models of sub-cellular biophysics and as models of ion and protein transport $[20]$.

The modified equation for RTP is

$$
d x(t)=\mathbf{v} d t+\sqrt{2 D} d w \text { where } \mathbf{v}=s v
$$

where $s= \pm 1$, changing signs according to a CTMC. Following their analysis [86], we define $P_{ \pm 1}(x, t)$ as the probability density for the particle to be at position $x$ with velocities $\pm v$ respectively. Also for convenience, we work with the dimensionless constants $\mathcal{D}=D \gamma / v^{2}$ and $l=L \gamma / v^{9}$. Here, the dynamics is assumed to take place in an unit interval $[-L,+L]$. Both $-L$ and $+L$ are absorbing. While similar to models in Chapter 2, the interval is different from interval in Chapter 2 where the interval used was $[0, L]$.

We define $E_{ \pm 1}(x)$ are the exit probabilities given the particle starts at $x$ with velocity \pm 1 exiting at $-l$.

\footnotetext{
${ }^{9}$ If $\gamma \rightarrow 0$, the effective parameters of the problem all go to zero. This is indicative of the fact the CTMC produces a singular perturbation $[105,115]$ in the Fokker-Planck equations. Such behavior is pervasive in these systems. For example, if we have $v=0$, the PDE needs to be reworked as that is yet another singular perturbation.
} 
For analyzing FPT properties for exit from a finite interval, the following two sets of equations involving exit probabilities ${ }^{10}$ are more pertinent

$$
\begin{gathered}
\mathcal{D} \frac{\partial^{2} E(x,+)}{\partial x^{2}}+\frac{\partial E(x,+)}{\partial x}-((E(x,+)-E(x,-))=0 \\
\mathcal{D} \frac{\partial^{2} E(x,-)}{\partial x^{2}}-\frac{\partial E(x,-)}{\partial x}+(E(x,+)-E(x,-))=0
\end{gathered}
$$

with boundary conditions $E(0, \pm)=1$ and $\left.E_{(} 0, \pm\right)=1$. Solutions to the above equation can be obtained in close form solutions and are as follows

$$
\begin{aligned}
& E(x,+)=\frac{e^{\alpha l}\left[(l-x)-\alpha \gamma_{-}\right]\left[(l-x)+\alpha \gamma_{+}\right]+\gamma_{+} e^{\alpha(l-x)}+\gamma_{-} e^{\alpha l}}{e^{\alpha l}\left[l+\frac{1}{\mathcal{D} \alpha}\right]+\left[l-\frac{1}{\mathcal{D} \alpha}\right]} \\
& E(x,-)=\frac{e^{\alpha l}\left[(l-x)+\alpha \gamma_{+}\right]\left[(l-x)+\alpha \gamma_{-}\right]-\gamma_{-} e^{\alpha(l-x)}-\gamma_{+} e^{\alpha l}}{e^{\alpha l}\left[l+\frac{1}{\mathcal{D} \alpha}\right]+\left[l-\frac{1}{\mathcal{D} \alpha}\right]}
\end{aligned}
$$

where

$$
\alpha^{2}=\frac{1}{\mathcal{D}^{2}}+\frac{2}{\mathcal{D}} \text { and } \gamma_{ \pm}=\frac{1}{2}\left(\frac{1}{\alpha} \pm \frac{1}{\mathcal{D} \alpha^{2}}\right)
$$

Similar expressions for $t_{+}(x, t)$ and $t_{-}(x, t)$, the mean first-passage time (to either boundary) for a particle that is at $x$ and is also in the $+(-)$ state can be calculated. The above equation can be contrasted with exit probability without the CTMC but with a constant $v$ as

\footnotetext{
${ }^{10}$ These equations can be derived from the coupled diffusion and CTMC evolution equations
} 


$$
E(x)=\frac{e^{-v x / D}-e^{-v L / D}}{1-e^{-v L / D}}
$$

We suggest potential roles for these models in the concluding part of the section. Next, we construct another model by relaxing the constant magnitude $v$ assumption, making it dependent on the state space. This leads to non-trivial exit path profiles. We argue that these models could better explain data from cognitive neuroscience experiments. Specifically, models of fluctuating drives may serve as models of dynamic conflict in certain conflict tasks.

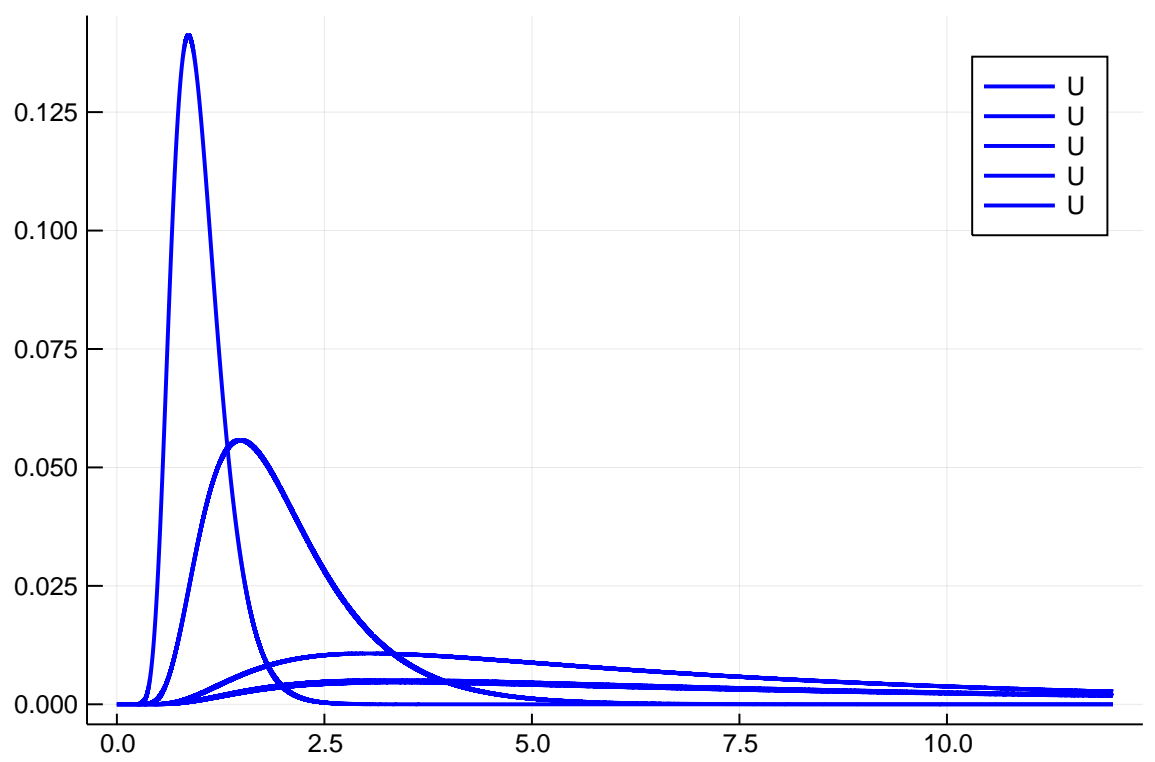

Figure 3.6: DDM with positive drift of increasing magnitude

$\mathrm{U}$ and L correspond to upper and lower decision boundaries. FPT densities in blue correspond to positive drift with increasing magnitude. $x$ axis denotes time and $y$ axis denotes first-passage time probability density. 


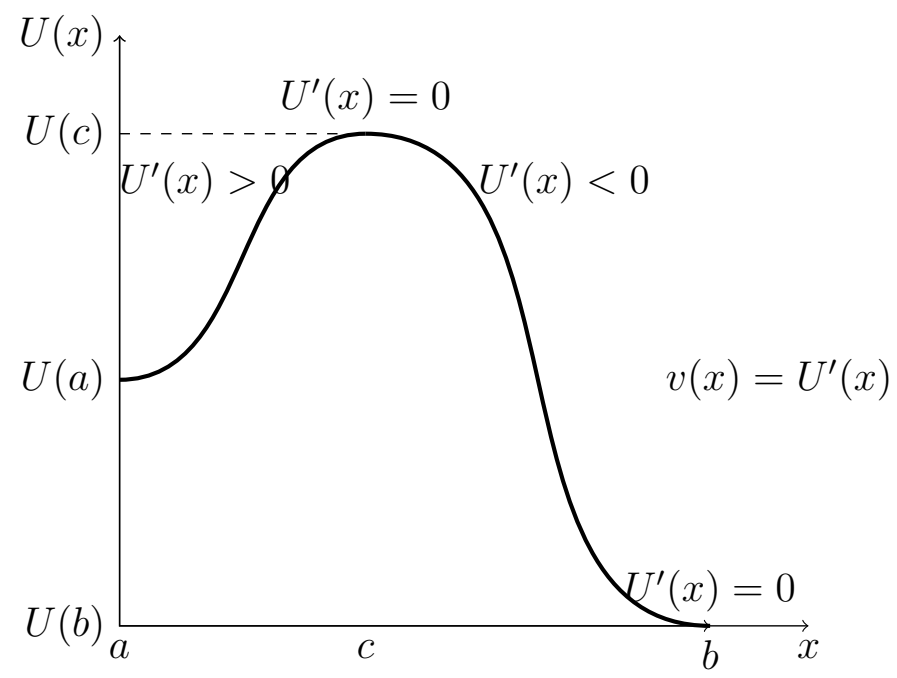

Figure 3.7: DDM with nonlinear deterministic drift

\subsubsection{Models with non-trivial deterministic drift}

The model presented previously had constant magnitude but stochastically fluctuating drift. In this model ${ }^{11}$ (see figure 3.2.3, the deterministic dynamics is made interesting by a state space dependent drift function. We begin with a simple one dimensional dynamical system with a state space evolution determined by an ordinary differential equation.

$$
\frac{d x}{d t}=-U^{\prime}(x) \text { with } x(0)=x_{0}
$$

where $U(x)$ is a potential function for the particle. The stable points of this system are exactly where $U^{\prime}(x)$ vanishes. Suppose there are three extrema of $U(x)$ at $a, b$, and $c$ with $a<c<b$. Further suppose $U(b)<U(a)<U(c)$ and $c$ is a local maxima and $a$ and $b$ are the system's local and global minima respectively. The deterministic system is stable to perturbations around $a$ and $c$ but any small

\footnotetext{
${ }^{11}$ There are several variants of this noisy escape from attractor problem in the literature. We closely follow Gardiner's analysis [51]
} 
perturbation away from $b$ in either direction pushes it towards one of the two minima, depending on the direction of perturbation.

To this system, we now add stochasticity modeled via a diffusion constant $D$ which is small in comparison to the scale of the deterministic dynamics, the system's stability is completely lost because the stochastic perturbation, in the long run can take the system to different parts of state space. The associated Fokker-Planck PDE is

$$
\frac{\partial p(x, t)}{\partial t}=\frac{\partial\left[U^{\prime}(x) p(x, t)\right]}{\partial x}+D \frac{\partial^{2} p(x, t)}{\partial x^{2}}
$$

One can perform calculations analogous to the previous model, to determine the probability of a particle starting a generic point $x$ going to either minima $a$ or $b$. We summarize the main results here but leave the details for the appendix. Again, the tools are similar and calculations approximate in nature. We follow [51] ${ }^{12}$ closely in the rest of this subsection. In the asymptotic limit $D \rightarrow 0$, the two probabilities $\pi_{a}\left(x_{0}\right)$ and $\pi_{c}\left(x_{0}\right)$, the probability that a particle at $x_{0}$ falls into $a$ or $c$ respectively.

Two cases are important because the singularly perturbed nature of the solutions. One in which $x_{0}$ is more than $\sim \sqrt{D}$ away from $c$; the other where $x_{0}$ is at a finite distance from $c$.

When $x_{0}$ is infinitesimally distant from $c$, the solution can be shown to be

$$
E_{c}=\frac{1}{2}\left\{1-\operatorname{erf}\left[\left(b-x_{0}\right) \sqrt{\frac{\left|U^{\prime \prime}(b)\right|}{D}}\right]\right\}
$$

where

\footnotetext{
${ }^{12}$ The calculations follow standard techniques and the interested reader can consult the book [51] section 9.1 for the derivations.
} 


$$
\operatorname{erf}(x)=\sqrt{\frac{\pi}{2}} \int_{0}^{x} d t e^{-t^{2}}
$$

When $x_{0}$ is at a finite distance, the solution is

$$
E_{c}=\frac{1}{U^{\prime}\left(x_{0}\right)} \sqrt{\frac{\left|U^{\prime \prime}(b)\right| D}{2 \pi}} \exp \left[\frac{U\left(x_{0}\right)-U(b)}{D}\right]
$$

with the splitting probability is only dependent on $c$. The probability of reaching $b$ in this limit is dependent on the particle jumping over the barrier $c$ with the other points being effectively infinite distance apart from each other in this approximation.

Having introduced the two models, we discuss applications of these models. In particular, we point out how the structure of models affords interpretation as models of psychological bias and codification of uncertainty in knowledge available.

\subsubsection{Applications}

We introduced two DDM extensions and demonstrated their analytical properties and calculated MFPT and expectation probabilities of exit. Typically measured experimentally like response time histograms may be identified with expressions for MFPT derived here. The extensions appeal to their dynamical systems nature and not to their statistical decision theoretic roots; they are plausible theoretical models whose empirical justification needs to be confirmed, but they are theoretically interesting and provide alternative generative models. This allowed us to employ newer class of SDE like stochastic hybrid models, piecewise-deterministic Markov models and multi-scale models [20]. Among the zoo of available interesting stochastic dynamical models available, the models discussed above were picked for their simplicity and novelty. To illustrate the use of these extensions, we con- 
sider their utility in biased decision making tasks. We add to discussions already present in the literature, specifically in [92] and suggest that the models introduced in this chapter may have relevance in explaining some of the documented results from their experiments.

A major objective in [92]was to identify neural correlates (fMRI) of prior knowledge in electrophysiological (EEG) and blood oxygenation dependent (BOLD) signals. These are multi-paradigm experiments as three different experimental tasks: electrophysiological, fMRI, and response time tasks are performed simultaneously. This allows a tighter integrating of data across different scales of the cognitive system.

In the experiment, subjects participated in an RT version of random dot motion task we discussed earlier in this chapter. In addition, cue indicating bias was presented next to the moving random dot images. The time it took for the subjects to decide whether the random dots moved to the right or to the left is the primary data for this task. They tested two models of prior knowledge. In one case, bias was encoded as changes in the starting point of the evidence accumulation process, the initial condition of a one-dimensional SDE; in the other, bias was encoded in a drift-rate that varied depending on the knowledge encoded. They conducted experiments to confirm their encoding of bias. While the DDM response times are measured like in conventional experiments, the subjects are inside a fMRI scanner so that their brain activities can also be measured simultaneously. In other experimental protocols, there is a possibility of even incorporating other imaging or recording techniques like EEG and MEG. Taken together, the collected data is capable of providing insights for different related disciplines in cognitive neuroscience.

Their results suggested that same regions of the brain were involved in both 
forms of prior knowledge. That is, when $v=0$

$$
E(x)=\frac{e^{-v x / D}-e^{-v L / D}}{1-e^{-v L / D}}
$$

$x$ is the starting point of the drift-diffusion process. It thus encodes information on prior knowledge. The choice probabilities are explicitly dependent on it. Similarly, changing $v$ can also change choice probabilities ${ }^{13}$.

Our models suggest additional ways in which prior knowledge (or norms [37] if we are looking at moral judgments) could be encoded. In many instances, instead of clear perceived evidence, one could envision a scenario where background knowledge is uncertain or if evidence is perceived to be continuously conflicting. Such uncertainty and confusion is exploited in various cognitive tasks like Stroop, Flanker and Simon tasks ${ }^{14}$. We discuss this in more detail later in this section. In such cases a RTP type DDM like the one discussed previously might be useful. Clearly, the functional form response time distributions for RTP-type model (see 3.7) and regular DDM (see equation 3.11) with bias as suggested by [92] are different; they suggest that the underlying neural and psychological processes are different.

In studies exploring the neural basis of pro-social behavior $[77,70]$, the response

\footnotetext{
${ }^{13}$ As we noted earlier, MPFT expressions derived in Chapter 2 can be used to generate synthetic data, if necessary. For example, DDM community uses very complicated hierarchical Bayesian models for fit response time data. the models introduced in this chapter could serve as alternative generative models. If the few parameter models of this chapter fit the data better, then it offers a simpler generative alternative to more complex statistical models.

${ }^{14}$ In Stroop tasks, the written word can conflict with the color in which it is rendered. For example, in experiments, subjects find it difficult to name the ink color of a color world if it is different from the ink color and the word.

In flanker tasks, flanking items can conflict with target items. The phenomena is that when stimuli are presented alongside other redundant stimuli, the original task becomes more difficult and error prone.

In Simon task, the location of the stimulus can conflict with the response.For example, subjects tend to perform better if the action to be taken and the direction of the stimuli are both in the same direction.
} 
time in subjects playing the public goods game ${ }^{15}$ were observed to be higher on average when considering altruistic pro-social behavior. Furthermore, as the game payoffs are changed, the subject response times change, and suggests a change in the parameters of the underlying DDM. The models of this chapter are candidate models of formal models of moral judgment [37].

In our model, the difference is due to the presence of a switching rate, the Poisson intensity $\gamma$. Since the DDMs presented in [92] and the fluctuating DDM, it would be interesting to see whether the brain regions encoding prior knowledge change to a new location, as uncertainty in knowledge is encoded in different regions of the brain. Testing this hypothesis about the location of uncertain prior knowledge might be an interesting complementary task to perform.

The second model which is based on multiple extrema offers a different mechanism for incorporating bias as the presence of two scales in the escape from attractor system creates dramatic differences in comparison to the conventional DDM case. As the analysis shows, MFPT and probabilities of transition to the exit points depend only on the value of the potential and its first and second derivatives at points $a, b$, and $c$ (see equations 3.23, 3.13 and 3.14).

Also noticeable in the escape from attractor model are two phases (see equations 3.13 and 3.14 ), one where the initial point $x_{0}$ is close to the local maxima $b$; the other where $x_{0}$ is a finite distance away from $b$. Instead of a variable drift rate model of bias as suggested in [92], this model encodes bias through a state dependent drift $U^{\prime}(x)$. The interpretation in this case is that the rate of conventional evidence accumulation rate $U^{\prime}(x)$ is state space dependent, slowing down in

\footnotetext{
${ }^{15}$ In a public goods game, players may contribute one's private coins towards a common pot. At the next stage, the pooled public tokens are multiplied by a factor, and evenly shared among the participants in the game. The Nash equilibrium of this game depends on the number of players and the public token multiplication factor.
} 
specific regions of state space corresponding to the extrema where $U^{\prime}(x)$ is close to 0 as the accumulation rate $v$ is a function of $U^{\prime}(x)$. Also, unlike conventional DDM, in this case, implicit in the model are two scales ${ }^{16}$, one, the order of the evidence accumulation process (deterministic dynamics); and the other, the scale set by the diffusion constant (noise). The neural correlates of such biases are expected to be different than the one found by [92] as the mechanism behind a subject's decision is different in the models presented in this chapter. Confirming this expectation via experiments like Forstmann et al [92] is an interesting direction for future work.

The two models may be useful in other settings too. Let us focus on the escape from attractor model. In a typical DDM, the relative frequency of choices, say 2AFC tasks, is of comparable magnitude. However, because noisy escape from attractors work differently (see equations 3.13 and 3.14), if we wish to guarantee rarity of one of the two choices, a model based on the escape from attractor is a better model than a conventional DDM.

Consider another scenario where transient evidence is presented at the start of the task in the form of a brief exposure. After an initial exposure, let us assume that only the internal processes dictate the dynamics. The initial exposure is going to set the value of $x_{0}$. Because of the strong nonlinearities present in the dynamics, the probability of a judgment depends only on the initial evidence point relative to the extrema $b$. We suggest that such scenarios potentially explain moral judgments tasks like norm violations [37] where framing effects and strongly internally embedded moral constraints are known to strongly determine judgment results.

\footnotetext{
${ }^{16}$ Unlike the models presented in Chapter 2 and in the CTMC based fluctuating drift model, the nontrivial deterministic dynamics modulated by $U(x)$ takes place at a different scale than the scale of the diffusion.
} 
The variants introduced here can also be thought as contributing to DDM based RT modeling of conflict tasks [135] like the Stroop task, Flanker task and Simon task. DDM used to explain RT characteristics observed in these tasks involve the use of time dependent drift functions $v(t)$, clearly leading to differences from simple model choice probabilities as described in equation 3.11. Both models introduced here encode conflicts either as a stochastic fluctuation term or in the form of static nonlinearity, which is different from conventional conflict DDM. As the neural basis or the specific psychological processes behind conflict tasks have not been identified, the models discussed in this chapter add to the pool of available candidate dynamic models for conflict tasks.

\subsection{From DDM to Ecological Rationality}

In Chapter 2, the same family of SDS was used to model escape from poverty traps. While the notion of traps has clear connections to the biological concept of viability, its connections to judgment and decision making in general, including models introduced in this chapter, needs to be clarified. In this section, we make these connections to viability via Giegerenzer's program $[52,53]$ around ecological rationality and its ancestry in systems ideas of satisficing and law of requisite variety discussed in Chapter 1.

DDM literature's original motivation in trying to explain speed-accuracy tradeoffs in cognitive tasks like 2AFC suggests natural connections between DDM and ecological, but the argument needs to be made. $2 \mathrm{AFC}$ and its variants mimic real world decision making tasks under time constraints. Without such constraints, almost all agents arrive at the right judgment. In other scenarios like one-choice reaction time tasks [104], it is the time it takes for the agent to reach a judgment 
or perform an action. Correspondence between DDM and ecological rationality could be established by appealing to the viability point of view espoused in chapter 1 using the correspondence between time to error or action as in [104] and persistence in a viable region.

While these models were developed within cognitive psychology, as an approach to dynamical psychological process modeling [85, 84], they could also be understood in terms of statistical decision theory, as decision processes that unfold over time. From this perspective, for an agent, it is sufficient to generate good enough decisions or judgments using available cognitive substrate. Furthermore, it suffices for decisions to be reached at times that match the temporal regularities and requirements of the environment; enabling the fastest possible judgments and decisions is not necessary. Both these points are in contrast to more traditional optimization oriented view of judgments and decision making. The perspective that normative evaluations of actions make sense only relative to the structure of the environment is associated with the concept of satisficing. And the demand that adaptive capability of materials should match the complexity of the environment is related to Ashby's law of requisite variety. These two systems ideas form the conceptual underpinnings of ecological rationality.

Giegerenzer's ecological rationality $[130,22]$ is offered an alternative to both the traditional conception of economic rationality and the error taxonomy based approach developed by Kahneman and Tversky. This ecological perspective disavows an optimization theoretic mathematical formalization of rationality, even if it is enriched by expressive computational models. It also rejects the error classification approach popularized by Kahneman and Tversky as scientifically unproductive because of its lack of reliance on computational models. Instead, ecological rationality proposes to develop computational and optimization based 
models that encode the structure and regularity of the environment; the normative criteria of what is rational is based on whether or not actions are good enough in an agent's environment. The fine tuning and constraint satisfaction based approach formulated in chapter 1 seeks to refine existing ecologically rational models by more explicitly and manifestly incorporating constraints of a temporal nature. Typically, models of ecological rationality [52] focus on providing alternatives to statistical decision theoretic conception of optimality based on environmental regularities. To the best of our knowledge, this proposed refinement of ecological rationality models via the conception of reliability articulated in Chapter 1 is an insight provided by the approach first introduced in Chapter 1.

The SDS underlying DDM is dynamical and process oriented by definition and computational via its focus on specific FPT scenarios. By associating the quality of decisions and judgments with various first-exit characteristics, a computational task completion models can be constructed, a suitable version of reliability is enabled, and robustness guaranteed via constraint satisfaction. While DDM has been cast as a stochastic optimal control problem $[12,11]$, viewing exitprobabilities and exit-time moments as constraints to be fine-tuned in modules has not been discussed in the literature. This fine tuning is achieved by restricting the parameters to enable good enough actions. This fine tuning without any explicit control modules makes in contradistinct with models of rationality using optimality as a criteria. In other words, both in ecological rationality literature $[130,22]$ and in this dissertation, rationality may be accomplished via optimization, via mere constraint satisfaction. As discussed earlier, DDM models are acknowledged to capture the idea of response time-accuracy trade-off. By associating this tradeoff with reliability and viability as conceptualized in Chapter 1, DDM can now be thought of as a model of ecological rationality. 
This conception of DDM as part of ecological rationality is also consistent with alternative views $[112,22]$ of Marr's Levels, which tries to emphasize noncomputational aspects of biological systems. In such viewpoints, understanding the architecture of cognitive function relative to biological behavior does not preclude the use of non-representationalistic dynamic models of processes. Use of DDMs in cognitive psychology as inferential models occludes their theoretical and conceptual value, and this insight was made clear and precise using the systems isomorphims from Chapter 1.

\subsection{Conclusion}

The two variants of DDM introduced above stand out in their novel use of nonlinear stochastic dynamical systems theory. To the best of our knowledge, the use of noisy escape from attractor scenarios in dynamical systems to model psychological processes is a novel contribution of this work. Also, the use of RTP as a DDM is also a contribution. As more bells and whistles are added to DDMs, inferring models from only response time distributions become increasingly intractable, a feature that is shared by all extant variants [66] and not just our models. A few natural steps can be taken to make these models more appealing, which we address in Chapter 4.

The novelty of the one dimensional models introduced in this chapter share many features of similar models introduced in Chapter 2 where they were used to study theoretically certain aspects of escape from poverty traps. Just like the models of Chapter 2, these models have not been discussed in DDM literature. While the models with stochastic drift were only recently introduced in micro nano physiology, the model with nonlinear gradient based drift is older and well known 
in physics, chemistry, biology and also in neuroscience. Despite its connections to neural spike initiation models, the model remained unused as a DDM. To the best of our knowledge, this work seems to be the first to introduce such models as models of dynamic judgment and decision making.

Similarly, while both ecological rationality literature and DDM literature independently discuss response-accuracy tradeoffs, the explicit connection between the two areas is made possible only by the notions of reliability, constraint satisfaction and fine tuning introduced in Chapter 1. Also, the models introduced here and others planned to be discussed in the future are all models used in micro and nano physiology. The model of escape from attractor discussed here has a potential role to play in escape from poverty traps as yet another model of poverty dynamics. Such crossovers between different unrelated disciplines is made possible by the notion of lifting introduced and discussed in chapter 1. The isomorphisms between models of poverty dynamics and models of judgment and decision making are not merely model isomorphisms; they are systems isomorphisms in that the theme of reliability, constraint satisfaction and fine tuning all carry over. This makes them semantically and pragmatically richer than simple transfer of mathematical knowledge about models.

Finally, at the very least, given the interesting features of the two models presented in this chapter, it is our hope that these models inspire ideas for new experiments or reanalysis of old experimental data in new light. 


\section{Appendix}

Given the relative magnitude of the noise and deterministic dynamics, WKB approximation ${ }^{17}$ suggests the following form for the solution

$$
p(x)=\mathcal{N} \exp [-U(x) / D]
$$

$\mathcal{N}$ is a normalization constant defined in equation 3.20 that is calculated order by order in perturbation theory to make sure that the probabilities add up to 1 .

A feature this model shares with the previous is that the nature of perturbation. Just as the CMTMC perturbation encountered previously, the addition of the diffusion constant $D$ is a singular perturbation; $D \rightarrow 0$ and $D=0$ are qualitatively different. Exact solutions are unavailable but approximate solutions using WKB approximations are available. We calculate them next. Assuming $(U(x)$ is sufficiently well-behaved, we can perform a a series expansion around the minima $a$ and $c$.

$$
\begin{aligned}
U(x) & \simeq U(a)+\frac{1}{2} U^{\prime \prime}(a)(x-a)^{2} & |x-a| \rightarrow 0 \\
& \simeq U(a)+\frac{1}{2} U^{\prime \prime}(a)(x-c)^{2} & |x-c| \rightarrow 0
\end{aligned}
$$

since $D$ is small, WKB ansatz gives us

\footnotetext{
${ }^{17}$ Originally used in quantum mechanics, Wentzell-Kramers-Brioullin approximation is a workhorse technique for calculating approximate solutions of (partial) differential equations involving multiple spatial and temporal scales
} 


$$
\begin{array}{rlrl}
p(x) & \simeq \mathcal{N}\left[-U(a) / D-\frac{1}{2} U^{\prime \prime}(a)(x-a)^{2} / D\right] & |x-a| \rightarrow 0 \\
& \simeq \mathcal{N}\left[-U(a) / D-\frac{1}{2} U^{\prime \prime}(c)(x-c)^{2} / D\right] & & |x-c| \rightarrow 0 \\
& \simeq 0 & & \text { everwhere else }
\end{array}
$$

with the normalization constant given by

$$
\mathcal{N}^{-1}=e^{-U(a) / D} \sqrt{2 \pi D / U^{\prime \prime}(a)}+e^{-U(c) / D} \sqrt{2 \pi D / U^{\prime \prime}(c)}
$$

Since we are working with asymptotic approximations $(D \rightarrow 0)$ the second term in the approximation can be dropped, leaving us with

$$
\begin{aligned}
p(x) & =\sqrt{\frac{U^{\prime \prime}(c)}{2 \pi D}} \exp \left[-\frac{1}{2} U^{\prime \prime}(c)(x-c)^{2} / D\right] & & |x-c| \sim \sqrt{D} \\
& =0 & & \text { everwhere else }
\end{aligned}
$$

showing that the global minima is the most stable. While correct, for large time $t \rightarrow \infty($ more precisely $\sim \exp (-$ const $/ D))$, the above approximation breaks down and the solution can cross the barrier maxima onto the other minima.

One can show that the time for the state at $a$, to reach $b$, the barrier is

$$
T(a \rightarrow b)=\pi\left[\left|U^{\prime \prime}(b)\right| U^{\prime \prime}(a)\right]^{-1 / 2} \exp \left[\frac{U(b)-U(a)}{D}\right]
$$

The above equation 3.23 can be generalized to arbitrary points $x$ and $y$ on the state space but as mentioned earlier, in the absence of closed form solutions, numerical evaluation requires other techniques. This will be pursued in a future work. 


\section{Inference for DDM}

In this appendix, we present inference procedure used for extracting paramters of the drift-diffusion model [131]. Consider the basic DDM with constant drift and diffusion constant ${ }^{18}$

$$
d x=v d t+s d W
$$

where $x$ is the evidence and $v$ is the evidence accumulation rate. $s d W$ is the standard Gaussian noise. This is for the 2AFC experiment. In this specific model, the agent accumulates evidence in favor of an action $C_{1}$ with a rate $v$, called the drift, meaning that in the case of a classical $2 \mathrm{AFC}$, the agent will also accumulate evidence against this evidence towards $C_{2}$. . Once the evidence crosses one of the two absorbing boundaries (the boundary $b$ positioned at a distance a corresponding to the action $C_{1}$ or the boundary $\neg b$. at position 0 corresponding to action $C_{2}$. This corresponds to upper decision boundary $\mathrm{U}$ and lower boundary L respectively. The corresponding action is selected after a delay $t_{0}$. The accumulation can start wherever at $z_{0} \in[0, a]$. We rescale $z_{0}$ to $w=z_{0} / a$.

The FPT density has a closed formula $\theta=\left\{a, v, w, t_{0}\right\}$

$$
\begin{gathered}
\text { Wiener }(t, b ; \mathbf{z})=\frac{\pi}{a^{2}} \exp \left(-v a w-\frac{v^{2}\left(t-t_{0}\right.}{2}\right) \sum_{k=1}^{\infty} k \exp \left(-\frac{k^{2} \pi^{2}\left(t-t_{0}\right)}{2 a^{2}}\right) \sin (k \pi w) \\
\theta^{*}=\underset{\theta}{\operatorname{argmax}} p(\mathbf{y} ; \theta)
\end{gathered}
$$

\footnotetext{
${ }^{18} \mathrm{~A}$ simplified notation is used for diffusion to better illustrate the steps in estimation.
} 


$$
=\underset{\theta}{\operatorname{argmax}} \frac{1}{S} \sum_{i=1}^{S} \frac{1}{T} \sum_{t=1}^{T} l\left(\mathbf{y}_{\mathbf{t}, \mathbf{s}} ; \theta_{\mathbf{s}}\right)
$$

$S$ correspond to the number of experimental subjects used in the trial. $T$ corresponds to the number of trials.

where the likelihood is

$$
l\left(\mathbf{y}_{\mathbf{t}, \mathbf{s}} ; \theta_{\mathbf{s}}\right)=\log \int W i e n e r\left(\mathbf{y}_{\mathbf{t}, \mathbf{s}} \mathbf{z}\right) p\left(\mathbf{z}_{\mathbf{t}} ; \theta_{\mathbf{s}}\right) d \mathbf{z}
$$

The data for such experiments come as pairs $\left(R T^{i}, C^{i}\right)$ corresponding to response time and the choice made. 


\section{Conclusion}

This dissertation makes the following contributions

1. In Chapter 1, using the concept of lifting, we generalized a specific notion of reliability and used it to define measures of resilience based on MFPT and FPT, and identified a constraint satisfaction pathway to guaranteeing reliable behavior that is in contrast to the more popular control theoretic approach. We generalized models of nano and micro physiology and applied it to two different domains: in mathematical sociology, to model poverty traps; and in mathematical psychology, to model bias and conflict in dynamic judgment and decision making tasks.

2. Chapter 2 introduced diffusion models to the theoretical literature on intragenerational social mobility and poverty traps and proposes new ecometric measures for urban sociologists.

3. Chapter 3 introduced new DDMs of judgment and decision making for modeling bias, uncertainty and conflicts and connected DDM and ecological rationality literature.

In this chapter, we suggest future work that builds upon the work discussed in chapters 1,2 and 3 .

In mathematical sociology, we argue for 
- higher dimensional escape from attractor scenarios as the next step towards developing more realistic models of poverty traps

- the use of Levy noises instead of just Brownian noise in models of intragenerational mobility

In mathematical psychology, we argue for

- higher dimensional models of escape from attractors to model multi-attribute decision making and judgment tasks

- the use of DDM is moral judgment and decision making

Additionally, we argue for

- the use of diffusion on rotation groups to model biological agents at the nano and micro scale

- the irreducibility of constraint satisfaction problems to simple optimization problems

- the need to develop better techniques to solve the inverse Fokker-Planck problem

In the remainder of this concluding chapter, we discuss future research directly inspired by the notion of reliability in SDS introduced in Chapter 1 and the work exemplifying this idea in Chapters 2 and 3. Future work mentioned here can be grouped together into three categories. The first category of work aims to improve upon the models used in Chapters 2 and 3. The second category of work aims to apply techniques used in Chapters 2 and 3 to model animal behavior at the meso and micro scale. The last category of work aims to develop mathematical tools 
necessary to extend MFPT analysis used in the dissertation to FPT analysis in more general settings, illuminating the role of fine constraint satisfaction and fine tuning in characterizing reliability of behavior.

\subsection{Summary of contributions and future work: Mathematical Soci- ology}

Chapter 2 models intragenerational mobility in the presence of poverty traps. While intragenerational mobility is not as well discussed in the economic literature as compared to intergenerational mobility, they are still discussed, but not in the context of poverty traps. The SDS approach sketched previously in Chaper 2 allows us to synthesize related domain knowledge and illustrate the importance of traps in understanding how actors escape poverty. Traditionally, wealth mobility and aspects of poverty are all studied separately in macroeconomics, microeconomics, economic sociology, urban sociology etc. The organizing principle of reliability helped us integrate ideas from different substantive disciplines, and propose new measures for use in analyzing sociological data.

SDS is given the following meaning. The state variable $x$ corresponds to wealth that evolves in time $x(t)$ and starts at $x_{0}$. The rate of growth of wealth is $v$ that is subjected to a Brownian shock represented by a diffusion constant $D$. The boundary of the domain is an unit interval say $[0,1]$ where 0 corresponds to a state of being trapped in permanent poverty and 1 corresponds to the actor escaping poverty.

While poverty traps are discussed as attracting fixed points of deterministic dynamics in equilibrium macroeconomic models, stochastic models of individual trajectory in poverty traps have not been discussed in economics or sociology. 
The work identifies the domain of dynamics $\mathcal{D}$ with a trap with escape out of the domain is considered to be desirable. The diffusion constant $D$ captures an ecological measure not directly interpretable as an economic measure, and may prove insightful to urban sociologists working with such ecometric measures. While policy makers are usually interested in wealth growth, the work points out how $D$ plays an important role in determining when social actors get out of poverty. A policy maker with sufficient resources could use such underappreciated policy levers to change $D$, fine tuning mobility to satisfy socially desirable constraints.

The models display a diversity of life histories for the social actors because of the diffusion constant and point out the role of chance in wealth accumulation dynamics; the divergence in life histories may arise due to luck, in the absence of explicit differences in the wealth accumulation rate. For an ensemble of actors, the first to escape from poverty and the last to escape from poverty all evolve as parts of the same dynamics. Identifying $D$ using data could be insightful for a better understanding of escape from poverty dynamics. From a policy maker perspective, the wealth growth rate can be controlled but $D$ can only be fine tuned. Such a tuning is one way to reduce divergence in outcomes of such social processes.

\subsubsection{Additional insights}

In deterministic dynamical systems, initial value conditions and boundary conditions structure the sample paths. These, along with the parameters of the dynamical systems, dictate its qualitative aspects of the associated global dynamics. Such phenomena appear in the models considered in this dissertation. One of the trivial and underappreciated aspects of stochastic systems is the sheer diversity of sample paths; diffusion models are no exception. One can demon- 
strate $[101,56]$ that the notion of a typical path to an absorbing boundary and MPFT makes sense only if path starts away from the boundary. The closer the starting point is to the boundary, the more diversity there is in the paths. The important point here is that the mechanisms are the same and only the initial starting point of the process is different. A similar aspect of stochasticity is the mere presence of other diffusion particles in shaping the first arrival time of a multiparticle system. When we consider an ensemble of large number of particles, the order statistics of first passage times are quite complex and varied. So the variances in particle-to-particle arrival times are an aspect of the rank ordered arrival time statistics $[101,136]$.

Pointing out such aspects of stochasticity may help illuminate some counterexamples to commonsense intuitions on social mobility dynamics among social scientists. For example, it is often assumed that differential rates of mobility for different demographic groups might be because of systemic institutional and environmental differences. That is, the drift and diffusion constants of the model are different for different socio-economic groups. But the above features of a simple diffusion model can capture a large amount of person-level variations without any differences in exposure to environmental mechanisms. A semi-analytical numerical exploration of these phenomena in the context of social and economic mobility is part of an on-going work and will be pursued in a future work.

Finally, the focus is on individual agents and not on collections of agents. In the future, we hope to develop mean field models of interacting agents and analyze their first-passage properties. Also important to include will be the roles of random environments, spatial and temporal heterogeneity of diffusion and drift parameters in shaping FPT models of mobility. Recent literature in comparative developmental economics suggest that the dynamics of poverty traps is non-trivial 
and highly variable; we suspect that stochastic dynamical systems and their associated exit phenomena, like the ones discussed in Chapter 2, have much to offer.

\subsection{Summary of contributions and future work: Mathematical Psy- chology}

Chapter 3 develops new drift-diffusion models of judgment and decision making. Traditionally, because of their association with explanatory models of response time data, proposed models have an incremental characteristic. The models are typically used as statistical models even though they purport to model actual evidence accumulation processes. A recent approach in cognitive psychology, modelbased cognitive neuroscience aspires to be integrative, utilizing models from different disciplines in neuroscience, electrophysiology and other disciplines. These approaches are still within cognitive psychology and neuroscience, in contrast to the ethological oriented approach espoused by this dissertation.

We found connections between DDM literature and ecological rationality literature by viewing an agent's psychological processes in the context of the environment in which a behavior takes place. Doing so frames such processes as dynamic analogs of static heuristics used by decision making agents. These heuristics are a result of encoding environmental regularities, resulting in biased heuristics. Simi-

larly, conflicting evidence or uncertainty in evidence may be perceived because of other kinds of environmental regularities. The general SDS approach presented in Chapter 1, and discussed in the context of DDM in Chapter 2, proposes models of evidence accumulation processes in the presence of uncertainty, conflicts and bias and is given the following meaning.

The state variable $x$ corresponds to the state of evidence that evolves in time 
$x(t)$ and starts at a starting state of evidence $x_{0}$. The rate of change of evidence $v$ is subjected to a noisy Brownian accumulation model represented by a diffusion constant $D$. The boundary of the domain is a unit interval say $[0,1]$ where 0 corresponds to one decision and 1 corresponds to another. In one model, $v$ is based on a CTMC and is identified with uncertainty in knowledge. In another model, $v$ is dependent on the state space $x$ and corresponds to intrinsic psychological bias.

While DDMs are discussed in the context of response accuracy trade-offs in time constrained decision making scenarios, their connections to ecological rationality, missed by the literature, and pointed out in Chapter 3, are first steps in establishing the relationship more formally. From this point of view, decisions and judgments are considered satisfactory or unsatisfactory relative to specific environmental state. The decision maker can be thought to fine tune the parameters like $v$ and $D$ based on learning over the life course to make sure a desired accuracy is reached.

The variants of DDMs provided here captures different aspects of these tradeoffs. In one model, a CTMC based drift is incorporated into the original model as an alternative model of uncertainty and conflicts. In another model, a nonlinear deterministic drift is used to model bias exhibited in such tasks. Both these models add to the literature on heuristics underlying judgment and decision making. The desired response times and accuracy of judgments are constrained by ecological requirements. The parameters of the models may be fine tuned by some learning processes that seek to satisfy constraints. This suggests the possibility that ecological rationality may be achieved with just fine tuning based constraint satisfaction. 


\subsubsection{Additional Insights}

The most rigorous way to confirm the utility of these two novel additions to DDM is to design new experiments by tweaking ones discussed here $[92,135]$. But in lieu of this, an alternative analysis with synthetic data could also be useful.

The parameter space of the original DDM is three dimensional: $x_{0}$, the starting point; $v$, the evidence accumulation rate; and $D$, the noise in the accumulation process. Our models add one or two additional parameters, depending on the form of either the stochastic switching rate $\gamma$, or $U(x)$. By systematically varying these parameters, one can generate synthetic data, which can be used to compare results from the original models.

Beyond the simplest of cases, the increasingly common approach to inference has been the use of non-parametric Bayesian methods. Scalable inference methods are difficult to employ without the use of advanced methods like variational inference [131]. In the future, we plan to collaboratively explore the use of such methods to statistically infer parameters of that model.

One of the attractive features of DDMs is that they are amenable to parameter estimation and model fit procedures. While the CTMC based DDM case maybe amenable through hierarchical modeling, the MFPT calculated using large deviation techniques are more difficult as they are based on rare-event analysis. Identifying approaches for model estimation and fit procedures is a direction that is being explored. Also necessary are very clear and specific proposals for experiments where sequential sampling models like the ones proposed here could be relevant. On that front, a preliminary step where synthetic data generated by simulations of these two models might be helpful. If such artificially generated data show novel signatures, one could look for them in existing datasets. For 
example, existing models all fit response time data to some variant of the standard DDM model with constant drift and diffusion coefficients. So, any signatures of deviation from the basic model family may be washed out because of model misspecification. If one were to fit response time data nonparametrically, then one could test whether such a nonparametrically fit data are comparable to the synthetic data produced from simulations.

The original home of these models is in biophysics, as models of ion and protein transport, electro-diffusion pheonmena and related processes [20, 115]. Thus, model based cognitive neuroscience provides a justification for transferring models used in neuronal and sub-neuronal biophysics and biochemistry into the realm of cognitive psychology. Inspired by this conceptual transfer of model families, other candidate models from such sources could be useful additions to DDM literature. Among them, escape from attractors in higher dimensions and narrow exits are two interesting phenomena that might have use as dynamical process models.

Unlike the simple escape from a one dimensional attractor scenario considered here, higher dimensions have potentially more interesting attractors. For example, in a future work, we plan on developing a two-dimensional escape from a stable limit cycle scenario where response time distributions are multimodal, providing an alternative to mixture model based explanation of multimodality. Other related models are based on higher dimensional systems where interface enabling exits out of the domain are narrow in comparison with the rest of the domain. Calculations of MPFT and exit distributions are similar in their use of WKB and other singular perturbation approximation techniques. Such models do belong to the class of SDS models presented in this dissertation. 


\subsection{Pragmatic value of these SDS systems isomorphisms}

Chapter 2 initiated the discussion of systems isomorphisms of stochastic dynamical systems (SDS) in mathematical sociology. The work revisits older literature on Markov models of occupational mobility and generalizes it to show how SDS can model intragenerational mobility and escape from poverty traps. By looking at exit-times and exit-probabilities of escape from such traps, it points out features of social dynamics, such as transients, which are often missed by equilibrium macroeconomic and macro-sociological models. Specifically, the parameters of the boundary value problem like $D$ and the boundary points may be identified with empirically observable quantities of interest to urban sociologists. Like other ecometric measures, these identified measures provide additional ways of thinking about interventions in such systems.

Chapter 3 used the same family of SDS in mathematical psychology The work looks at literature on drift-diffusion models (DDM) of time constrained judgment and decision making. Inspired by models of escape from attractor and stochastic switching dynamics in simple neurophysiological processes, it proposes extensions of DDM, again illustrating the role of exit-times and exit-probabilities. These models could serve as not only as null reference models for experiments in cognitive psychology and motivate new experiments, but also suggest alternative psychological mechanisms. As discussed in chapter 3, these models suggest alternative ways of encoding uncertainty and bias in dynamic psychological processes.

While both applications make sense as contributions in their respective disciplines, they can be viewed as good examples of the lifted concept of fine tuning based constraint satisfaction, originally identified in physiology. In poverty trap models, parameters available for fine tuning corresponded to ecometric measures. 
Their precise value was mapped to the diffusion constant $D$ of the SDS. In psychological DDMs, fine tuning corresponded to keeping the parameters of SDS like $D$ and $v$ in order to produce an observed response time for judgment or decision.

As we argued in Chapter 1, isomorphisms have not only conceptual value but they also provide value to scientific disciplines. Chapters 2 and 3 exemplifies how this contributes to respective discipline-centric scientific enterprise. Typically, establishment of isomorphisms identify gaps within disciplinary boundaries and suggest advances in the form of new models not considered before by discipline centric epistemic community. In many cases, the relations between phenomena in different disciplines, and between related areas of research within a discipline can be made more obvious with this approach. For example, a natural model of escape from poverty traps(Chapter 2) and the connections between DDM and ecological rationality(Chapter 3) was made possible by the isomorphism.

This dissertation was based on how a specific family of SDS endow a kind of reliability in agents in the presence of exogenous and endogenous stochasticity. This starting point in micro and nano physiology, with all its biosemiotic significance, is what makes this approach to systems science biology-centric. We used mathematical ideas of first-exits in stochastic dynamical systems to model this reliability in the presence of stochasticity. Having established a mathematical model of a specific kind of biological reliability, this approach postulates their existence in unrelated disciplines with non-interacting epistemic communities. In this research project, the presence of such models in two disciplines, albeit framed in disciplinary language, is a vindication of this systems perspective. 


\subsubsection{Gaps and connections in scientific literature identified by SDS isomorphisms}

In sociology, an existing body of work was identified pertaining to models of occupational mobility over the life course, and mobility of actors within firms and organizations. In all these cases, sociological inquiry was focused on systematic differences between actors belonging to different socio-economic and demographic groups, on the mean time for reaching higher occupational status, and reasons for differential times for different groups. If we frame these questions from a policy maker perspective, tuning the social environment to guarantee more equality in outcomes is related to questions pertaining to reliability -the primary focus of this dissertation. While the literature $[14,42,8]$ employs discrete time Markov chains (DTMC) or continuous time Markov chains (CTMC) in their analysis, diffusion models were not included in their stochastic models. Similarly, while economic sociologists use diffusion models to study wealth growth, the literature [10] is not interested in first-passage time questions as the questions are more aligned towards macroeconomics and macro-sociology, and ignore temporal features of transients. And finally, while urban sociologists are aware of the dynamics of poverty and the presence of traps, the empirical focus of research on urban poverty is more statistically oriented. The simple models of chapter 2 contribute to these questions by offering complementary generative and mechanistic models.

In psychology, literature surrounding response time experiments where judgment and decision response times are explained using noisy evidence accumulator based models. Although a wide variety of DDM were proposed since their original introduction, their focus on explaining actual response time data likely prevented them from using deductive models like the ones we proposed in chapter 3. From a 
fine-tuning perspective, the reliability of response times has obvious connections to satisficing. However, in spite of being very influential, the DDM community has not made connections to ecological rationality literature. Concurrently, while foraging models are used in ecological rationality literature [22, 130], they have mostly ignored DDM as possible models of satisficing. The systems oriented approach discussed in chapter 3 offers unique contributions to this research literature, again by finding rigorous theoretical connections between related sub-fields like ecological rationality and time constrained judgment and decision making.

\subsubsection{Gaps and connections in the mathematical literature identified by SDS isomorphisms}

The key analytical tool in SDS is the Fokker-Planck partial differential equation boundary value problem (FPE) . As discussed previously, both FPT PDF and MFPT satisfy a PDE that involves the Fokker-Planck operator. Even for simple cases discussed in this dissertation, while MFPT could be derived analytically, the PDF associated with the more important quantity of FPT, is intractable analytically. Also, from a reverse engineering point of view, the inverse problem where one specifies a PDF for FPT, and asks for an appropriate Fokker-Planck operator that satisfies constraints, is rarely discussed in the mathematical literature. In addition, while FPEs associated with simple SDS state spaces are well-understood, the correspondence between higher dimensional SDS with non-trivial state spaces and the associated PDE operator seems to have not been dealt with comprehensively in the literature. To provide a model instantiation of biological functional requirement seems to require new ways of thinking about old mathematical ques- 
tions. Other related issues also require filling gaps in the mathematical literature ${ }^{1}$.

In chapter 3 , an escape from attractor scenario was used to model bias. A natural question is whether such models can be generalized to higher dimensional dynamical systems with non-trivial attractors. Such models are not only useful for DDM, they are potentially useful also for developing more realistic models of poverty traps. FPE for such systems involving multiple scales become very complicated and the theory behind generalized escapes does not exist, but needs to be developed. Similarly, while nonlinear dynamics on non-trivial state spaces like rigid body dynamics necessary for animal behavior applications are well-known, FPT and MFPT analysis of rotational and rigid body diffusions, as suggested by the biological motivations of this dissertation, is another gap that needs filled.

Finally, the conceptual argument behind this dissertation was to provide a constraint satisfaction based worst-case analysis of certain kinds of reliability. However, because the mathematics required to develop such a worst-case analysis is absent, we relied on a MFPT based analysis. The proposed fine tuning without control modules approach requires that we are able to demonstrate the construction of SDS that satisfy viability constraints. This requires a solution to the inverse problem that starts with a FPT PDF, provides a Fokker-Planck operator, and makes sure the problem is not ill-posed. To the best of our knowledge,

\footnotetext{
${ }^{1}$ While conventional methods for numerically solving PDE, like finite element methods, work well for low dimensions, as the dimensions of the PDE increase, the grids associated with finite elements fall to the curse of dimensionality. This is true for other approaches for obtaining solutions like Monte Carlo methods. Also, while finite element methods and Monte Carlo methods are well-established for simple state spaces like the unit-interval, PDE on non-trivial manifolds, like the ones required for modeling animal behavior, are not so well-established. On the other hand, MFPT equations are equations about expectation values of probability distributions. Typically, they are easier to calculate than the full FPT probability density function (PDF). Although FPT PDF is available in closed form for simple one-dimensional processes, only numerical approximations are available for general diffusion processes. Therefore, in many situations, MFPT is used as a surrogate quantity and is therefore much more prevalent than FPT analysis.
} 
literature on such inverse problems is sparse.

\subsection{Scientific explorations suggested by these systems isomorphisms}

Chapter 2 developed simple diffusion with drift to illustrate time characteristics of intragenerational mobility. While similar out-of-equilibrium models are prevalent in the literature $[1,122]$, models better informed by developmental economics, social geography and economic sociology may contribute substantively to the discourse on intragenerational social processes. In Section 1.1, we present potential future work inspired by these possibilities.

Chapter 3 cast DDM through the lens of first-passage time analysis and extended it to model biased decision making. As discussed there, mathematical psychologists have gone beyond two-alternative forced choice experiments to model different kinds of psychological processes involving decisions that occur under noisy evidence accumulation scenarios. Inspired by such extensions, we briefly outline additional variants that employ aspects of stochastic dynamics not currently employed in the literature in Section 1.2.

In this section, we briefly discuss a variety of scientific questions that are a direct outgrowth of questions suggested by implications of systems isomorphisms, and natural continuation of discipline focused inquiries conducted in Chapters 2 and 3. In most instances, the extensions sketched here are merely an enlargement of model class of SDS discussed earlier.

In some instances, extensions to models come in the form of addition of random jumps to SDS models discussed in this dissertation. For example, instead of just drift and diffusion terms, we also include a jump term, making it a Levy pro-

cess. Similarly, we could generalize the models to multidimensional drift-diffusion 
models of escape from poverty dynamics. Finally, the pertinent example of biological behavior extends the one-dimensional analysis to state spaces of non-trivial topology like spheres and the space of rotations of a rigid body.

\subsubsection{More general models of escape from poverty}

In chapter 2, models of intragenerational poverty were developed based on simple drift-diffusion models. The resulting work was intended to be a proof of concept illustration of the utility of temporal characteristics of social processes in understanding social and economic pathways out of poverty over the life course. While useful, poverty dynamics are prone to shocks, resulting in abrupt changes in wealth. Also, wealth dynamics of more standard economic models maybe useful in certain instances. Both these features can be modeled by adding more structure into the stochastic process.

For example, to model more general forms of wealth shocks, the original model must be replaced with

$$
d \mathbf{x}=F(\mathbf{x}) d t+H(\mathbf{x}) d L
$$

where $H(\mathbf{x})$ corresponds intensity of a Levy noise [97]. While this term makes the SDE analytically intractable, it is a natural next step implementable through simulations.

Another limitation of the models discussed in chapter 2 and its new version presented above (4.1) is that they are not derived from microeconomic and macroeconomic foundations. It is desirable to derive models from economic and sociological first principles and study their FPT characteristics. It is possible to derive such SDE from first principles [9]. One such equation, which is a special case of 
the Levy noise based SDE is

$$
d X=r(s) X d t+\sigma(s) d w
$$

where $s$ is an exogenous Markov process, $X$ is the wealth and $w$ is standard Brownian noise. It can be shown that the stationary distribution of such a stochastic process is a fat tailed distribution, making it an attractive model of observed inequalities in wealth. FPT and MFPT analysis of such models as discussed for simple models in chapter 2 might be an interesting future research direction.

One aspect that is under-explored is their finite time property and associated FPT, as most of in economics relies on standard equilibrium assumptions of macroeconomic models. We expect finite time characteristic to play a role in understanding economic geographic processes. Another aspect that is often ignored are effects of spatial extents and the use of models from chapter 2 and those based on equation (4.1 and 4.2) to study neighborhood level processes [61].

\subsubsection{More variants of DDMs}

As discussed in chapter 3, DDMs and their variants were deployed to explain time constrained judgment and decision tasks under noisy evidence accumulation scenarios. The psychological state of a DDM can be attributed to not only single neuron activity, but also to neural assemblies and emergent macrostate dynamics like EEG activity. Inspired by goals of model-based cognitive neuroscience, we argue that the models discussed in Chapter 3 are also useful models of collective electrophysiological processes.

Also discussed earlier, cross-pollination between ecological rationality literature and DDM literature is absent. A reliability perspective offered in this dis- 
sertation provides a concrete mathematical example of rationality without optimality [23]. Constructing of clear model theoretically motivated by making the connection more explicit are needed.

Continuing our presentation of variants of DDM, other models based on stochastic dynamical systems [115] can be introduced. As discussed at the end of the last Chapter 3, rather than use a continuous noise accumulation process, one can introduce jumps reflecting sudden availability of evidence alongside existing continuous accumulation. This leads to Levy process based DDM. Another interesting class of models is one where boundaries themselves are drift-diffusion processes. Processes where the boundary thresholds are set by other dynamic psychological processes are good situations where such models could prove useful and consistent with processes in nano and micro physiology.

Similarly, in higher dimensions, PDF of FPT in systems with non-trivial attractors exhibit multimodality and localization not seen in simple situations. They suggest alternatives to existing DDMs. These extensions are not just theoretically interesting; they suggest a more careful reanalysis of existing data with these new model families in mind. For example, Levy noise based evidence accumulation models, like in equation (4.1), can lead to fat-tailed response times [97]. Escape times on non-trivial attractors [38] display oscillations in FPT distribution. Also, when response time distributions are fitted using DDM, the models are assumed to be of the simple drift-diffusion kind. If the data were really fat-tailed or show multimodality, Levy noise or higher dimensional models would better characterize the data. 


\subsubsection{Animal Behavior}

A natural continuation of applications of ideas from Chapter 1 are discussions of models of animal behavior that uses first-passage time characteristics to understand reliability of behavioral primitives. The mathematical techniques required to address stochastic dynamical systems of extended objects is surprisingly nontrivial. Also, significant steps are required to model stochasticity in such constrained state spaces as even simple extensions of Levy processes to non-linear manifolds become mathematically very difficult $[3,83]$ and requires advances not made in the literature.

Here, we sketch two important directions as first steps: one model uses a nonlinear oscillator as a template for a fixed action pattern; the other uses a motion of a thin rigid rod as a template for a startle. Models presented here can be reworked to be good prototypes of agents in stochastic environments.

Fixed Action Patterns Locomotion or repetitive animal movements are exemplars of fixed action patterns. Under idealized conditions, these movements are enabled by microcircuits that span both the muscular-skeletal system and the nervous system. Similar movement is possible even in organisms without a nervous system. Such movements are reliable even in the presence of stochasticity in the environment. Continuing with the premise of this dissertation, we break away from an optimal control based model of FAP and suggest a constraint satisfaction approach using FPT characteristics.

A direct and general approach is possible using results from [18]. In this case, the system corresponds to a $d$-dimensional SDE on $\mathbb{R}^{d}$

$$
d \mathbf{x}=F(\mathbf{x}) d t+\sqrt{\epsilon} G(\mathbf{x}) d W
$$


where the noise free dynamical system has a stable periodic solution. By doing a phase-amplitude decomposition, one can derive a separate SDE for amplitude and phase variables. Using techniques similar to ones used in Chapters 2 and 3, FPT and MFPT for the amplitude to exceed a certain threshold can be calculated. A very recent work [19] uses this to study synchronization of oscillators under the presence of various kinds of noise.

Startle and Escape Response Startles and escape responses are known for their high reliability and are quintessential behavioral primitives documented by ethologists. In such behaviors, an ideal response is one that with high certainty, avoids certain regions of state space; and with high certainty, reaches other regions of state spaces. Both requirements can be cast as a kind of probabilistic constraint satisfaction. All this makes it ideal extensions of MPFT and FPT based reliability analysis discussed in this dissertation.

A typical organism is extended in space and behavioral states are specified by both center of mass coordinates and body coordinates. Although unrealistic, an idealized version of an agent is a rigid body in three dimensional space. For example, a typical change in state of a rigid body from $\mathbf{x}$ to $\mathbf{x}^{\prime}$ in space can be represented as

$$
\mathbf{x}^{\prime}=\mathbf{R} \mathbf{x}+\mathbf{b}
$$

where $\mathbf{R}$ corresponds to a rotation and $\mathbf{b}$ to a translation. Now if we were to include stochasticity, then the equation needs to be adapted to look like

$$
\mathbf{x}^{\prime}=\mathbf{R} \mathbf{x}+\mathbf{b}+\text { noise }
$$


making it a stochastic differential equation in 6-dimensions. Solutions to FokkerPlanck equation is such spaces are non-trivial but available, requiring a numerical approach in all but the simplest of cases [35].

As a first step, we begin with an idealized agent fixed in space but now the agent is an extended rigid rod. The state space of such a system can be specified by spherical polar coordinates $(\theta, \phi)$. Suppose the viable region for this agent is a small polar cap $\theta \leq \theta_{0}$ at the north pole of this sphere. Also suppose the agent's orientation at initialization starts at the south pole $\theta(0)=\pi$. In the presence of spherically diffusive noise, FPT and MFPT characteristics for the state to enter the viable north polar cap can be calculated [24] via solutions to FPE on the sphere $\mathcal{S}^{2}$.

\subsection{Mathematical explorations suggested by these systems isomor- phisms}

This dissertation formulates reliability as a fine tuning and constraint satisfaction property. However, a conceptual argument alone is insufficient; a rigorous mathematical demonstration of the concept's irreducibility of constraint satisfaction to optimization is necessary. Among the various reliability measures, FPT characteristics played an extensive role in our analysis. For example, unimodality of FPT PDF is one such characteristic. The presence or absence of unimodality in SDS has consequences for reliability, and necessary and sufficient conditions for unimodality ${ }^{2}$ are of importance for the program outlined in this dissertation. In the remainder of this section, we discuss two potentially useful future research directions inspired by these motivations.

\footnotetext{
${ }^{2}$ Even when unimodality can be established, bounds on magnitude of variance must is another important dimension along which fine tuning may be necessary.
} 


\subsubsection{Duality between Optimization and Constraint Satisfaction}

This first exploration arose out of a need to mathematically justify the status of constraint satisfaction. In typical presentations of how optimality is achieved in natural systems, primary status is given to optimization, and constraint satisfaction is afforded only a secondary more peripheral role. This has had consequences especially in model of cognition based on optimality principles [80]. In this literature surrounding Bayesian models of cognition, a Marrian approach to cognition is based on Bayesian decision theory, ignoring the role of constraint satisfaction.

Typical claims defending the choice to ignore constraint satisfaction invoke arguments based on the presence of dual problems. It argues that even if the problem is framed in terms of constraints, there always exist dual problems that do not involve constraints. The following argument is part of ongoing work that counters this commonly held view.

Linear programming formulations of optimization problems are one of the easiest to discuss and is used to explain the premise of this project. Consider a linear program

$$
\begin{aligned}
\operatorname{minimize} & \mathbf{c}^{T} \mathbf{x} \\
\text { subject to } & \mathbf{A x} \preceq \mathbf{b}
\end{aligned}
$$

A dual program can be defined where $\boldsymbol{\lambda}$ is the dual variable. 


$$
\begin{array}{cl}
\operatorname{maximize} & -\mathbf{b}^{T} \boldsymbol{\lambda} \\
\text { subject to } & \mathbf{A}^{T} \boldsymbol{\lambda}+\mathbf{c}=0 \\
\boldsymbol{\lambda} \succeq 0
\end{array}
$$

Although simple to state, finding solutions to these programs can be computationally hard, sometimes falling into the class of $\mathcal{N} \mathcal{P}$ Hard problems. One approach to finding solutions to these problems is to create duals to the original problems and solving them instead. Under certain conditions, solutions to the original problem $\mathbf{x}_{*}$ and $\boldsymbol{\lambda}_{*}$ are identical. When they are not, a duality gap is said to exist. It is possible to convert hard to compute inequalities to easier dual programs with only equalities or pure optimization with constraint problems via duality transformations. In what follows, this notion of duality gap and constraint free linear programs are important.

The primacy of constraint satisfaction can be confronted using duality theorems. One could argue that any sequence of constraint satisfaction problems can be converted to pure optimization problems. Given that duality gaps are non-zero for all but certain special families of programs and that bounds on those gaps cannot be bounded for all programs, all conversions to pure optimization problems are approximate.

Even if duality gaps are zero, duality transformations are computationally nontrivial; this has consequences for systems for questions of biological reliability and viability. Biological agents are usually resource constrained, designed to satisfice. In ethologically salient scenarios, the agent may not have the resources to per- 
form non-trivial duality transformations to environmental cues that are naturally codified as constraint satisfaction problems.

To illustrate this point, consider a linear program formulation [16] which has a gap free solution. The costliest step in the computation of a dual is a matrix inversion. If one considers a specific matrix inversion problem, say Strassen's algorithm, the complexity scales as $\mathcal{O}\left(n^{2.8}\right)$, or the Winograd algorithm with complexity $\mathcal{O}\left(n^{2.4}\right)$. Since matrix inversion can be done exactly, one can analyze specific approximate methods for matrix inversion by comparing the approximate solution with the actual solution. This discrepancy can be quantified by using some matrix norm. If we demand that the matrix inversion takes place in sub-quadratic time, then the accuracy of the resulting matrix inverse suffers. The difference between an actual solution and an approximate solution induces a duality gap in an otherwise gap free problem.

To the best of our knowledge, the occurrence of resourced constraints induced gaps and their applications to game theory has not been discussed in the literature.

\subsubsection{Inverse problem approach for general stochastic dynamical sys- tems}

The second exploration arose out of attempts to address the indirect way in which FPT analysis was used in the dissertation. The dissertation, motivated by a reverse engineering approach to understanding certain kinds of reliability in agents, used FPT analysis of SDS in different state spaces to demonstrate this. While the arguments made in chapter 1 require PDF of FPT, only MPFT based analysis is presented in chapters 2 and 3. Also, to establish the constructive (algorithmic) nature of fine tuning based constraint satisfaction, an reverse formulation is required. Rather than solve the FPE to derive the PDF $u$, what is necessary is to 
derive a Fokker-Planck operator $L_{\mathbf{x}}^{*}$ from a given $\hat{u}$.

As introduced in chapter 1 , the specific definition of reliability introduced in this dissertation is discussed around general solutions to the following boundary value problem [115]

$$
u(x, T)=P\{\tau<T \mid \mathbf{x}(t=0)=x\}
$$

where $u(x, T)$ is the cumulative probability of a particle starting at $x$ to reach the boundary before time $T . \mathcal{D}$ is the domain and $B d(\mathcal{D})$, its boundary. The associated random variable is represented by $\tau . \mathcal{L}$ is the Fokker-Planck operator. The associated PDE for the cumulative probability is given by

$$
\begin{aligned}
& \frac{\partial u(\mathbf{x}, t, T)}{\partial t}+\mathcal{L} u(x, t, T)=0 \quad \text { for } \quad x \in \mathcal{D}, t<T \\
& u(x, t, T)=1 \quad \text { for } \quad x \in B d(\mathcal{D}), t<T \\
& u(x,, t, T)=0 \quad \text { for } \quad x \in B d(\mathcal{D})
\end{aligned}
$$

If the work in this dissertation were to be used, to model behaving agents to satisfy certain constraints, solutions to inverse problems that start with PDF of $\tau$, seeking an appropriate $\mathcal{L}$ needs to be constructed.

Most of the model cases we have examined in this dissertation have very simple FPT distribution structure. In some instances, the distributions can be multimodal or have fat tails [57]. When this happens, MFPT becomes meaningless and FPT based characterization of behavior becomes inadequate. The necessary and sufficient conditions for FPT distribution to be unimodal have consequences for understanding biological reliability and is part of future work. 


\subsection{Conclusion}

In this dissertation, a systems approach of identifying isomorphisms among phenomena in disparate fields was shown to be useful in scientific inquiry. Specifically, it introduced novel models in mathematical sociology, as models of intragenerational mobility; and mathematical psychology, as models of drift-diffusion model for judgment and decision making. By connecting models to a certain kind of reliability, via first-passage time analysis, the importance of fine tuning based constraint satisfaction was demonstrated.

Fine tuning and control mechanisms are two different ways to generate optimal behavior. Constraint satisfaction seems to be as important as optimization. In certain scenarios, constraint satisfaction is more relevant, especially in the version of reliability presented in this work. Finally, much insight stands is gained from viewing higher biological behavior as a physiological process. While the way we lifted physiological concepts and elevated them to systems ideas is quiet common, it has not been formalized as such. This way of looking at systems ideas holds promise.

Pertinent questions in specific disciplines often do catalyze progress in the tools used for scientific inquiry, and motivate progress in related disciplines; physics for much of the 20th century and then biology in the present century are apt examples. We consider the use of systems isomorphisms to be akin to such efforts. 


\section{Bibliography}

[1] F. Abergel, H. Aoyama, B.K. Chakrabarti, A. Chakraborti, and A. Ghosh. Econophysics of Agent-Based Models. New Economic Windows. Springer International Publishing, 2013.

[2] Yves Achdou, Francisco J. Buera, Jean-Michel Lasry, Pierre-Louis Lions, and Benjamin Moll. Partial differential equation models in macroeconomics. Philosophical Transactions of the Royal Society A: Mathematical, Physical and Engineering Sciences, 372(2028):20130397, 2014.

[3] David Applebaum. Lévy processes in stochastic differential geometry. In Lévy processes, pages 111-137. Springer, 2001.

[4] F Gregory Ashby and James T Townsend. Varieties of perceptual independence. Psychological review, 93(2):154, 1986.

[5] W.R. Ashby. An Introduction to Cybernetics. 1963.

[6] A. Banerjee and E. Duflo. Poor Economics: A Radical Rethinking of the Way to Fight Global Poverty. PublicAffairs, 2012.

[7] Abhijit Banerjee, Dean Karlan, Robert Darko Osei, Hannah Trachtman, and Christopher Udry. Unpacking a multi-faceted program to build sustainable income for the very poor. Working Paper 24271, National Bureau of Economic Research, February 2018. 
[8] D.J. Bartholomew. Stochastic Models for Social Processes. Wiley series in probability and mathematical statistics. Wiley, 1978.

[9] Jess Benhabib and Alberto Bisin. Skewed wealth distributions: Theory and empirics. Journal of Economic Literature, 56(4):1261-91, December 2018.

[10] Yonatan Berman, Yoash Shapira, and Moshe Schwartz. A fokkerplanck model for wealth inequality dynamics. EPL (Europhysics Letters), 118(3):38004, may 2017.

[11] Sebastian Bitzer, Hame Park, Felix Blankenburg, and Stefan Kiebel. Perceptual decision making: drift-diffusion model is equivalent to a bayesian model. Frontiers in Human Neuroscience, 8:102, 2014.

[12] Rafa Bogacz, Eric Brown, Jeff Moehlis, P. Holmes, and Jonathan D. Cohen. The physics of optimal decision making: a formal analysis of models of performance in two-alternative forced-choice tasks. Psychological review, 113 4:700-65, 2006.

[13] Josh Bongard and Hod Lipson. Automated reverse engineering of nonlinear dynamical systems. Proceedings of the National Academy of Sciences, 104(24):9943-9948, 2007.

[14] R. Boudon. Mathematical Structures of Social Mobility. Progress in mathematical social sciences. Jossey-Bass, 1973.

[15] S. Bowles, S.N. Durlauf, and K. Hoff. Poverty Traps. Princeton University Press, 2011. 
[16] S. Boyd, S.P. Boyd, L. Vandenberghe, and Cambridge University Press. Convex Optimization. Berichte über verteilte messysteme. Cambridge University Press, 2004.

[17] Thomas Leopold Bram Hogendoorn and Thijs Bol. Divorce and the growth of poverty gaps over the life course: A risk and vulnerability approach. Technical report, DIAL, 022019.

[18] P. Bressloff and J. MacLaurin. A variational method for analyzing stochastic limit cycle oscillators. SIAM Journal on Applied Dynamical Systems, $17(3): 2205-2233,2018$.

[19] Paul C. Bressloff and James MacLaurin. Synchronization of stochastic hybrid oscillators driven by a common switching environment. Chaos: An Interdisciplinary Journal of Nonlinear Science, 28(12):123123, 2018.

[20] Paul C. Bressloff and James N. Maclaurin. Stochastic hybrid systems in cellular neuroscience. The Journal of Mathematical Neuroscience, 8(1):12, Aug 2018.

[21] Paul C. Bressloff and Jay M. Newby. Stochastic models of intracellular transport. Rev. Mod. Phys., 85:135-196, Jan 2013.

[22] Henry Brighton. Rationality without optimality: Bounded and ecological rationality from a marrian perspective, Aug 2018.

[23] Henry Brighton. Rationality without optimality: Bounded and ecological rationality from a marrian perspective. In Riccardo Viale, editor, Routledge Handbook of Bounded Rationality, To Appear. Routledge, UK, 2019. 
[24] David R. Brillinger. A particle migrating randomly on a sphere. Journal of Theoretical Probability, 10(2):429-443, Apr 1997.

[25] Rodney A Brooks. Intelligence without representation. Artificial intelligence, 47(1-3):139-159, 1991.

[26] Theodore Bullock. How do Brains Work?: Papers of a Comparative Neurophysiologist. Contemporary Neuroscientists. Birkhäuser Boston, 2013.

[27] Mario Bunge. Method, model and matter, volume 44. Springer Science \& Business Media, 2012.

[28] Jerome R. Busemeyer, Sebastian Gluth, Jrg Rieskamp, and Brandon M. Turner. Cognitive and neural bases of multi-attribute, multi-alternative, value-based decisions. Trends in Cognitive Sciences, 23(3):251 - 263, 2019.

[29] J. Cartailler, Z. Schuss, and D. Holcman. Geometrical effects on nonlinear electrodiffusion in cell physiology. Journal of Nonlinear Science, 27(6):19712000, Dec 2017.

[30] D. Centola. How Behavior Spreads: The Science of Complex Contagions. Princeton analytical sociology series. Princeton University Press, 2018.

[31] Raj Chetty, David Grusky, Maximilian Hell, Nathaniel Hendren, Robert Manduca, and Jimmy Narang. The fading american dream: Trends in absolute income mobility since 1940. Science, 356(6336):398-406, 2017.

[32] Raj Chetty and Nathaniel Hendren. The impacts of neighborhoods on intergenerational mobility i: Childhood exposure effects*. The Quarterly Journal of Economics, 133(3):1107-1162, 2018. 
[33] Raj Chetty and Nathaniel Hendren. The impacts of neighborhoods on intergenerational mobility ii: County-level estimates*. The Quarterly Journal of Economics, 133(3):1163-1228, 2018.

[34] Raj Chetty, Nathaniel Hendren, Patrick Kline, and Emmanuel Saez. Where is the land of opportunity? the geography of intergenerational mobility in the united states *. The Quarterly Journal of Economics, 129(4):1553-1623, 2014.

[35] G.S. Chirikjian and A.B. Kyatkin. Harmonic Analysis for Engineers and Applied Scientists: Updated and Expanded Edition. Dover Books on Mathematics. Dover Publications, 2016.

[36] D. Collins, J. Morduch, S. Rutherford, and O. Ruthven. Portfolios of the Poor: How the World's Poor Live on \$2 a Day. Princeton University Press, 2010 .

[37] Molly J. Crockett. How formal models can illuminate mechanisms of moral judgment and decision making. Current Directions in Psychological Science, $25(2): 85-90,2016$.

[38] K. Dao Duc, Z. Schuss, and D. Holcman. Oscillatory survival probability: Analytical and numerical study of a non-poissonian exit time. Multiscale Modeling ES Simulation, 14(2):772-798, 2016.

[39] Clintin P. Davis-Stober, Nicholas Brown, Sanghyuk Park, and Michel Regenwetter. Recasting a biologically motivated computational model within a fechnerian and random utility framework. Journal of Mathematical Psychology, 77:156 - 164, 2017. 
[40] A. Deaton. The Great Escape: Health, Wealth, and the Origins of Inequality. Princeton University Press, 2013.

[41] P. Demeulenaere. Analytical Sociology and Social Mechanisms. Cambridge University Press, 2011.

[42] A. Diekmann and P. Mitter. Stochastic Modelling of Social Processes. Elsevier Science, 2014.

[43] Jan Drugowitsch. Fast and accurate monte carlo sampling of first-passage times from wiener diffusion models. Scientific reports, 6:20490, 2016.

[44] Elena A. Erosheva, Ross L. Matsueda, and Donatello Telesca. Breaking bad: Two decades of life-course data analysis in criminology, developmental psychology, and beyond. Annual Review of Statistics and Its Application, 1(1):301-332, 2014.

[45] Jean-Claude Falmagne and Jean-Paul Doignon. Stochastic evolution of rationality. Theory and Decision, 43(2):107-138, Sep 1997.

[46] Birte U. Forstmann and Eric-Jan Wagenmakers. Model-based cognitive neuroscience: A conceptual introduction. In B.U. Forstmann and E.J. Wagenmakers, editors, An introduction to model-based cognitive neuroscience, pages 49-70. Springer, 2015.

[47] B.U. Forstmann, R. Ratcliff, and E.-J. Wagenmakers. Sequential sampling models in cognitive neuroscience: Advantages, applications, and extensions. Annual Review of Psychology, 67(1):641-666, 2016. PMID: 26393872.

[48] B.U. Forstmann and E.J. Wagenmakers. An Introduction to Model-Based Cognitive Neuroscience. Springer New York, 2015. 
[49] Yoël Forterre, Jan M Skotheim, Jacques Dumais, and Lakshminarayanan Mahadevan. How the venus flytrap snaps. Nature, 433(7024):421, 2005.

[50] Noah E. Friedkin. Social cohesion. Annual Review of Sociology, 30(1):409425, 2004.

[51] C.W. Gardiner. Handbook of Stochastic Methods: For Physics, Chemistry and the Natural Sciences. Springer series in synergetics. Springer, 1985.

[52] G. Gigerenzer, R. Hertwig, and T. Pachur. Heuristics: The Foundations of Adaptive Behavior. Oxford University Press, 2015.

[53] Gerd Gigerenzer. Axiomatic rationality and ecological rationality. Synthese, Jul 2019.

[54] Julijana Gjorgjieva, Guillaume Drion, and Eve Marder. Computational implications of biophysical diversity and multiple timescales in neurons and synapses for circuit performance. Current Opinion in Neurobiology, 37:4452, 2016. Neurobiology of cognitive behavior.

[55] Julijana Gjorgjieva, Guillaume Drion, and Eve Marder. Computational implications of biophysical diversity and multiple timescales in neurons and synapses for circuit performance. Current Opinion in Neurobiology, 37:4452, 2016. Neurobiology of cognitive behavior.

[56] Aljaz Godec and Ralf Metzler. First passage time distribution in heterogeneity controlled kinetics: Going beyond the mean first passage time. 6:20349, 022016. 
[57] Aljaž Godec and Ralf Metzler. First passage time distribution in heterogeneity controlled kinetics: going beyond the mean first passage time. Scientific reports, 6:20349, 2016.

[58] H. Goldstein, C.P. Poole, and J.L. Safko. Classical Mechanics. Pearson, 2013.

[59] Deborah M Gordon. Ant encounters: interaction networks and colony behavior, volume 1. Princeton University Press, 2010.

[60] Deborah M Gordon. The evolution of the algorithms for collective behavior. Cell systems, 3(6):514-520, 2016.

[61] Bryan S. Graham. Identifying and estimating neighborhood effects. Journal of Economic Literature, 56(2):450-500, June 2018.

[62] Corina Graif and Robert J. Sampson. Spatial heterogeneity in the effects of immigration and diversity on neighborhood homicide rates. Homicide Studies, 13:242-260, 2009.

[63] S. Grillner and A.M. Graybiel. Microcircuits: The Interface Between Neurons and Global Brain Function. Dahlem workshop reports. MIT Press, 2006.

[64] P. Hedstrom. Dissecting the Social: On the Principles of Analytical Sociology. Cambridge University Press, 2005.

[65] D.R. Hofstadter and Fluid Analogies Research Group. Fluid Concepts and Creative Analogies: Computer Models of the Fundamental Mechanisms of Thought. Penguin Press Science Series. Penguin Books, 1998. 
[66] D Holcman and Z Schuss. Time scale of diffusion in molecular and cellular biology. Journal of Physics A: Mathematical and Theoretical, 47(17):173001, apr 2014.

[67] David Holcman and Rafael Yuste. The new nanophysiology: regulation of ionic flow in neuronal subcompartments. Nature Reviews Neuroscience, 16(11):685, 2015.

[68] J.H. Holland, K.J. Holyoak, R.E. Nisbett, and P.R. Thagard. Induction: Processes of Inference, Learning, and Discovery. Bradford books. MIT Press, 1989.

[69] Crawford S Holling. Resilience and stability of ecological systems. Annual review of ecology and systematics, 4(1):1-23, 1973.

[70] Cendri A. Hutcherson, Benjamin Bushong, and Antonio Rangel. A neurocomputational model of altruistic choice and its implications. Neuron, $87(2): 451-462,2015$.

[71] K. Immelmann and C. Beer. A Dictionary of Ethology. Harvard University Press, 1989.

[72] M.O. Jackson. The Human Network: How Your Social Position Determines Your Power, Beliefs, and Behaviors. Knopf Doubleday Publishing Group, 2019.

[73] Eric J. Johnson and Roger Ratcliff. Chapter 3 - computational and process models of decision making in psychology and behavioral economics. In Paul W. Glimcher and Ernst Fehr, editors, Neuroeconomics (Second Edi- 
tion), pages 35 - 47. Academic Press, San Diego, second edition edition, 2014 .

[74] O. Kallenberg. Foundations of Modern Probability. Probability and Its Applications. Springer New York, 2002.

[75] Niels Keiding. Event history analysis. Annual Review of Statistics and Its Application, 1(1):333-360, 2014.

[76] Marc Keuschnigg, Niclas Lovsjö, and Peter Hedström. Analytical sociology and computational social science. Journal of Computational Social Science, 1(1):3-14, Jan 2018.

[77] I. Krajbich, B. Bartling, Todd Hare, and Ernst Fehr. Rethinking fast and slow based on a critique of reaction-time reverse inference. Nature Communications, 012015.

[78] Ian Krajbich and Antonio Rangel. Multialternative drift-diffusion model predicts the relationship between visual fixations and choice in value-based decisions. Proceedings of the National Academy of Sciences, 108(33):13852$13857,2011$.

[79] Thibault Lagache and David Holcman. Quantifying intermittent transport in cell cytoplasm. Phys. Rev. E, 77:030901, Mar 2008.

[80] Brenden M. Lake, Tomer D. Ullman, Joshua B. Tenenbaum, and Samuel J. Gershman. Building machines that learn and think like people. Behavioral and Brain Sciences, 40:e253, 2017. 
[81] P. Langley, H.A. Simon, G.L. Bradshaw, and J.M. Zytkow. Scientific Discovery: Computational Explorations of the Creative Processes. MIT Press, 1987.

[82] Thomas Lecuit and L. Mahadevan. Morphogenesis one century after on growth and form. Development, 144(23):4197-4198, 2017.

[83] M. Liao. Invariant Markov Processes Under Lie Group Actions. Springer International Publishing, 2018.

[84] R Duncan Luce. Four tensions concerning mathematical modeling in psychology. Annual Review of Psychology, 46(1):1-27, 1995.

[85] R.D. Luce. Response Times: Their Role in Inferring Elementary Mental Organization. Oxford Psychology Series. Oxford University Press, 1991.

[86] Kanaya Malakar, V Jemseena, Anupam Kundu, K Vijay Kumar, Sanjib Sabhapandit, Satya N Majumdar, S Redner, and Abhishek Dhar. Steady state, relaxation and first-passage properties of a run-and-tumble particle in one-dimension. Journal of Statistical Mechanics: Theory and Experiment, 2018(4):043215, apr 2018.

[87] D. Marr. Vision: A Computational Investigation Into the Human Representation and Processing of Visual Information. Mit Press. MIT Press, 2010.

[88] Miller McPherson. A Blau space primer: prolegomenon to an ecology of affiliation. Industrial and Corporate Change, 13(1):263-280, 022004.

[89] Miller McPherson, Lynn Smith-Lovin, and James M Cook. Birds of a feather: Homophily in social networks. Annual Review of Sociology, 27(1):415-444, 2001. 
[90] J. Monod and A. Wainhouse. Chance and Necessity: An Essay on the Natural Philosophy of Modern Biology. Penguin, 1997.

[91] S.L. Morgan, D.B. Grusky, and G.S. Fields. Mobility and Inequality: Frontiers of Research in Sociology and Economics. Studies in social inequality. Stanford University Press, 2006.

[92] Martijn J. Mulder, Eric-Jan Wagenmakers, Roger Ratcliff, Wouter Boekel, and Birte U. Forstmann. Bias in the brain: A diffusion model analysis of prior probability and potential payoff. Journal of Neuroscience, 32(7):2335$2343,2012$.

[93] Louis Narens. Multimode utility theory. Journal of Mathematical Psychology, 75:42 - 58, 2016. Special Issue in Honor of R. Duncan Luce.

[94] M. Newman. Networks. OUP Oxford, 2018.

[95] Daniel O'Brien, Robert J. Sampson, and Christopher Winship. Ecometrics in the age of big data: Measuring and assessing 'broken windows' using largescale administrative records. Sociological Methodology, 45:101-147, 2015.

[96] P. Paoletti and L. Mahadevan. A proprioceptive neuromechanical theory of crawling. Proceedings of the Royal Society B: Biological Sciences, 281(1790):20141092, 2014.

[97] Ccile Penland and Brian D Ewald. On modelling physical systems with stochastic models: diffusion versus levy processes. Philosophical Transactions of the Royal Society A: Mathematical, Physical and Engineering Sciences, 366(1875):2455-2474, 2008. 
[98] T. Piketty and A. Goldhammer. Capital in the Twenty-First Century. Harvard University Press, 2017.

[99] Rafael Polana, Ian Krajbich, Marcus Grueschow, and Christian C. Ruff. Neural oscillations and synchronization differentially support evidence accumulation in perceptual and value-based decision making. Neuron, 82(3):709 $-720,2014$.

[100] Chetty R, Stepner M, Abraham S, and et al. The association between income and life expectancy in the united states, 2001-2014. JAMA, 315(16):17501766, 2016.

[101] M. Ralf, R. Sidney, and O. Gleb. First-passage Phenomena And Their Applications. World Scientific Studies in International Economics. World Scientific Publishing Company, 2014.

[102] Roger Ratcliff. A theory of memory retrieval. Psychological review, 85(2):59, 1978.

[103] Roger Ratcliff. Decision making on spatially continuous scales. Psychological review, 125(6):888, 2018.

[104] Roger Ratcliff and Hans P. A. Van Dongen. Diffusion model for one-choice reaction-time tasks and the cognitive effects of sleep deprivation. Proceedings of the National Academy of Sciences, 108(27):11285-11290, 2011.

[105] S. Redner. A Guide to First-Passage Processes. A Guide to First-passage Processes. Cambridge University Press, 2001.

[106] Michel Regenwetter and Yung-Fong Hsu. Stochastic process models of preference change. In The Oxford Handbook of Public Choice, Volume 1. 
[107] Samuel Rudy, Alessandro Alla, Steven L Brunton, and J Nathan Kutz. Data-driven identification of parametric partial differential equations. arXiv preprint arXiv:1806.00732, 2018.

[108] Samuel H. Rudy, Steven L. Brunton, Joshua L. Proctor, and J. Nathan Kutz. Data-driven discovery of partial differential equations. Science Advances, $3(4), 2017$.

[109] Robert J. Sampson. Neighborhood Effects, Causal Mechanisms, and the Social Structure of the City, pages 227-250. Cambridge University Press, Cambridge and New York, 2011.

[110] Robert J. Sampson. Individual and community economic mobility in the great recession era: The spatial foundations of persistent inequality. Economic Mobility: Research and Ideas on Strengthening Families, Communities and the Economy, pages 261-287, 2016.

[111] Robert J. Sampson and Jeffrey D. Morenoff. Durable Inequality: Spatial Dynamics, Social Processes and the Persistence of Poverty in Chicago Neighborhoods, pages 176-203. Princeton University Press, Princeton, NJ, 2006.

[112] Larissa K. Samuelson, Gavin W. Jenkins, and John P. Spencer. Grounding cognitive-level processes in behavior: The view from dynamic systems theory. Topics in Cognitive Science, 7(2):191-205, 2015.

[113] Hayden Schaeffer. Learning partial differential equations via data discovery and sparse optimization. Proceedings of the Royal Society A: Mathematical, Physical and Engineering Sciences, 473(2197):20160446, 2017. 
[114] Michael Schmidt and Hod Lipson. Distilling free-form natural laws from experimental data. Science, 324(5923):81-85, 2009.

[115] Z. Schuss. Theory and Applications of Stochastic Processes: An Analytical Approach. Applied Mathematical Sciences. Springer New York, 2009.

[116] Z. Schuss, A. Singer, and D. Holcman. The narrow escape problem for diffusion in cellular microdomains. Proceedings of the National Academy of Sciences, 104(41):16098-16103, 2007.

[117] G.M. Shepherd and S. Grillner. Handbook of Brain Microcircuits. Oxford University Press, 2017.

[118] S.J. Shettleworth. Cognition, Evolution, and Behavior. Oxford University Press, 2010.

[119] H.A. Simon. The Sciences of the Artificial. The MIT Press. MIT Press, 1996.

[120] H.A. Simon. Models of Discovery: and Other Topics in the Methods of Science. Boston Studies in the Philosophy and History of Science. Springer Netherlands, 2012.

[121] Herbert A Simon. Rational choice and the structure of the environment. Psychological review, 63(2):129, 1956.

[122] S. Sinha, A. Chatterjee, A. Chakraborti, and B.K. Chakrabarti. Econophysics: An Introduction. Physics textbook. John Wiley \& Sons, 2010.

[123] Philip L. Smith. Stochastic dynamic models of response time and accuracy: A foundational primer. Journal of Mathematical Psychology, 44(3):408 463, 2000. 
[124] Philip L Smith. Diffusion theory of decision making in continuous report. Psychological Review, 123(4):425, 2016.

[125] Philip L Smith and Elaine A Corbett. Speeded multielement decisionmaking as diffusion in a hypersphere: Theory and application to doubletarget detection. Psychonomic bulletin \& review, pages 1-36, 2018.

[126] Philip L Smith and Roger Ratcliff. An introduction to the diffusion model of decision making. In B.U. Forstmann and E.J. Wagenmakers, editors, An introduction to model-based cognitive neuroscience, pages 49-70. Springer, 2015.

[127] B. F. Spencer and L. A. Bergman. On the numerical solution of the fokkerplanck equation for nonlinear stochastic systems. Nonlinear Dynamics, 4(4):357-372, Aug 1993.

[128] D.A.W. Thompson. On Growth and Form. Number v. 1 in Dover Books on Biology Series. Dover Publications, 1992.

[129] N. Tinbergen. On aims and methods of ethology. Zeitschrift fr Tierpsychologie, 20(4):410-433.

[130] Peter M. Todd and Henry Brighton. Building the theory of ecological rationality. Minds and Machines, 26(1):9-30, Mar 2016.

[131] Moens Vincent and Zenon Alexandre. Recurrent auto-encoding drift diffusion model. bioRxiv, 2018.

[132] Barbara Webb. Chapter 1: Using robots to understand animal behavior. volume 38 of Advances in the Study of Behavior, pages 1 - 58. Academic Press, 2008. 
[133] Ryan Webb. Dynamic constraints on the distribution of stochastic choice: Drift diffusion implies random utility. Available at SSRN, 2226018, 2013.

[134] Ryan Webb. The (neural) dynamics of stochastic choice. Management Science, 65(1):230-255, 2019.

[135] Corey N. White, Mathieu Servant, and Gordon D. Logan. Testing the validity of conflict drift-diffusion models for use in estimating cognitive processes: A parameter-recovery study. Psychonomic Bulletin $\&$ Review, 25(1):286301, Feb 2018.

[136] S. Bravo Yuste and Katja Lindenberg. Order statistics for first passage times in one-dimensional diffusion processes. Journal of Statistical Physics, 85(3):501-512, Nov 1996.

[137] Martin Zwick. Elements and relations. Springer Publications. In preparation, 2020. 